\title{
Perturbation expansions of signal subspaces for long signals
}

\author{
VLADIMIR NEKRUTKIN
}

Singular Spectrum Analysis and many other subspacebased methods of signal processing are implicitly relying on the assumption of close proximity of unperturbed and perturbed signal subspaces extracted by the Singular Value Decomposition of special "signal" and "perturbed signal" matrices. In this paper, the analysis of the largest principal angle between these subspaces is performed in terms of the perturbation expansions of the corresponding orthogonal projectors. Applicable upper bounds are derived. The main attention is paid to the asymptotic case when the length of the time series tends to infinity. Results concerning conditions for convergence, the rate of convergence, and the main terms of proximity are presented.

AMS 2000 SUBJeCt ClAssifications: Primary 65G99, 65F30; secondary 65F 15 .

Keywords AND PHRASEs: Signal subspace methods, Perturbation expansion, Asymptotic analysis, Singular Spectrum Analysis.

\section{INTRODUCTION}

Though the first publication on the subspace-based methods of signal processing traditionally runs back to [1], the explosive development of these methods occurred during the last 30 - 35 years. In numerous scientific and engineering areas from climatology [2] and meteorology [3] to acoustics [4], from petroleum geology [5, pp 41-51] to marine sciences [6], from human [7] and animal [8] population dynamics to noise reduction problems [9], many researchers independently proposed similar "signal-subspace" ideas and applied them to time series of their own interest.

Omitting details that are specific and important for each version of the method, the general scheme of the signal-subspace approach can be explained as follows. Consider a time series $\mathrm{F}_{N}=\left(x_{0}, \ldots, x_{N-1}\right)$ treated as "signal". (Note that $\mathrm{F}_{N}$ can be one-dimensional or multidimensional, real-valued or complex-valued.) This series is linearly transformed into a certain $L \times K$ "signal matrix" $\mathbf{H}$. (The entries of $\mathbf{H}$ can also be either real-valued or complex-valued.) It is supposed that the signal $\mathrm{F}_{N}$ and the transformation $\mathrm{F}_{N} \mapsto \mathbf{H}$ are such that $d \stackrel{\text { def }}{=} \operatorname{rank} \mathbf{H}<\min (L, K)$ and the linear space spanned by the columns of matrix $\mathbf{H}$ contains important information about the series $\mathrm{F}_{N}$. The latter space is called signal subspace.

In terms of the "covariance" matrix $\mathbf{A} \stackrel{\text { def }}{=} \mathbf{H H}^{*}$ this means that the eigenspace $\mathbb{U}_{0}$ corresponding to zero eigenvalue of the matrix $\mathbf{A}$ is not degenerate and that we are interested in the orthogonal complement $\mathbb{U}_{0}^{\perp}$ of $\mathbb{U}_{0}$. Note that the matrix $\mathbf{H}$ must be a certain structured matrix. Depending on the data at hand and on the problem under study, Hankel, Toeplitz, block-Hankel, and other similar matrices are used in practice.

Assume now that the perturbed series $\mathrm{F}_{N}(\delta)=\mathrm{F}_{N}+\delta \mathrm{E}_{N}$ is observed rather than the unperturbed series $\mathrm{F}_{N}$, where $\mathrm{E}_{N}=\left(e_{0}, \ldots, e_{N-1}\right)$ is a "noise series" and $\delta$ stands for a formal perturbation parameter. Thus, instead of the "signal matrix" $\mathbf{H}$ we observe the perturbed matrix $\mathbf{H}(\delta)=\mathbf{H}+\delta \mathbf{E}$, where the "noise matrix" $\mathbf{E}$ is constructed from the series $\mathrm{E}_{N}$ in the same manner as $\mathbf{H}$ is built from the series $\mathrm{F}_{N}$. Note that generally there is no a priori assumption on the structure of the noise series. For example, the origin of $\mathrm{E}_{N}$ can be random or deterministic.

Consider the Singular Value Decomposition (briefly, SVD) of $\mathbf{H}(\delta)$. If $\delta$ is small, then continuity considerations show that the linear space $\mathbb{U}_{0}^{\perp}(\delta)$ spanned by $d$ leading left singular vectors of this SVD can serve as an approximation of $\mathbb{U}_{0}^{\perp}$. Thus we can expect that useful (though approximate) information about the signal $\mathrm{F}_{N}$ can be extracted from $\mathbb{U}_{0}^{\perp}(\delta)$ with the help of a certain special technique. Since the linear space $\mathbb{U}_{0}^{\perp}(\delta)$ is spanned by $d$ leading eigenvectors of the matrix $\mathbf{A}(\delta) \stackrel{\text { def }}{=} \mathbf{H}(\delta) \mathbf{H}^{*}(\delta)$, this procedure is usually formulated in terms of the eigendecomposition of the perturbed "covariance" matrix $\mathbf{A}(\delta)$.

These or similar ideas under different names (for example, Eigenvector Filtering [6], Extended Empirical Orthogonal Functions [3], Karhunen-Loève Time Series Analysis [7], Singular System Analysis [10], Dynamic Principal Component Analysis [11]) or without any special names are used in many publications. The so-called Singular Spectrum Analysis (whether treated as in [12] or as in [13]) has the same origin.

The most known example of this scheme is the following. Suppose that a one-dimensional series $\mathrm{F}_{N}$ is governed by some minimal Linear Recurrent Formula (briefly, LRF)

$$
x_{n}=\sum_{k=1}^{d} b_{k} x_{n-k}, \quad n \geq d
$$


of order $d$. Transform $\mathrm{F}_{N}$ into the $L \times K$ Hankel "trajectory" matrix $\mathbf{H}$ with rows $\left(x_{j}, \ldots, x_{K+j-1}\right)$, where $j=0, \ldots, L-1$ and $L+K=N+1$. If $\min (L, K)>d$, then $\operatorname{rank} \mathbf{H}=d=$ $\operatorname{dim} \mathbb{U}_{0}^{\perp}$ and $\operatorname{dim} \mathbb{U}_{0}=K-d>0$.

The knowledge of $\mathbb{U}_{0}^{\perp}$ (or, equivalently, of $\mathbb{U}_{0}$ ) provides an essential information about the series $\mathrm{F}_{N}$. In particular, if we consider Schubert basis $\mathcal{Y}=\left(Y_{1}, \ldots, Y_{L-d}\right)$ of the linear space $\mathbb{U}_{0}$ (see [14] or $[13$, Ch. 5] for relations between this basis and LRFs), then the first element $Y_{1}$ of $\mathcal{Y}$ determines the minimal LRF governing the series $\mathrm{F}_{N}$. Finding the roots of the related characteristic polynomial is the usual goal in practical investigations.

There is a large number of publications dedicated to methods and algorithms for estimation of these roots by the use of the linear space $\mathbb{U}_{0}^{\perp}(\delta)$ in the case when $\mathrm{E}$ is a whitenoise series (see, for example, chapters 11 and 13 in [15]). Methods named as MUSIC and ESPRIT are presently the most popular. Standard references for the origins of these approaches are [16] and [17]. A modern description of ESPRIT together with the first-order perturbation analysis of its performance can be found in [18].

Let us discuss the aim, the technique, and the results of the paper. The main goal is to estimate the difference between $\mathbb{U}_{0}^{\perp}$ and $\mathbb{U}_{0}^{\perp}(\delta)$ in the case of long signals.

We describe both unperturbed $\mathbb{U}_{0}^{\perp}$ and perturbed $\mathbb{U}_{0}^{\perp}(\delta)$ signal subspaces by their orthogonal projectors $\mathbf{P}_{0}^{\perp}$ and $\mathbf{P}_{0}^{\perp}(\delta)$, respectively. The spectral norm (also known as the operator norm) $\|\cdot\|$ is used to measure the difference between these projectors. (Spectral norm of a matrix can be defined as its maximal singular value.) Since the dimension of $\mathbb{U}_{0}^{\perp}$ and $\mathbb{U}_{0}^{\perp}(\delta)$ is the same, the norm $\left\|\mathbf{P}_{0}^{\perp}(\delta)-\mathbf{P}_{0}^{\perp}\right\|$ is the sine of the largest principal angle between the linear spaces $\mathbb{U}_{0}^{\perp}(\delta)$ and $\mathbb{U}_{0}^{\perp}$. For example, this follows from [19, Ch. $3 \S 39]$.

To estimate this norm from above we use the perturbation expansions of $\mathbf{P}_{0}^{\perp}(\delta)$. These expansions (see formulas (2.2) and (2.4) of Section 2) are valid under the condition $|\delta|<\delta_{0}$ for some positive $\delta_{0}$ and can be straightforwardly deduced from the classical monograph [20, Ch. II $\S 3]$. It follows from (2.4) that the projector $\mathbf{P}_{0}^{\perp}(\delta)$ is continuous in $\delta$ as $|\delta|<\delta_{0}$. In other words, if $|\delta|<\delta_{0}$ then $\mathbb{U}_{0}^{\perp}(\delta)$ can be continuously traced back to $\mathbb{U}_{0}^{\perp}$.

Suitable upper bounds for $\left\|\mathbf{P}_{0}^{\perp}(\delta)-\mathbf{P}_{0}^{\perp}\right\|$ lead to the solution of several problems related to the proximity of perturbed and unperturbed signal subspaces in the case of long signals. Solving these problems, we restrict ourselves to realvalued signals governed by LRFs.

More precisely, we consider an infinite signal series $\mathrm{F}=$ $\left(x_{0}, x_{1}, \ldots, x_{n}, \ldots\right)$ which is governed by a minimal linear recurrent formula of order $d$. As it was already described, finite segments $\mathrm{F}_{N}=\left(x_{0}, x_{1}, \ldots, x_{N-1}\right)$ of the series $\mathrm{F}$ are transformed into signal $L \times K$ Hankel matrices $\mathbf{H}$, where $L$ depends on $N$. Thus the signal subspace $\mathbb{U}_{0}^{\perp}$ with the related projector $\mathbf{P}_{0}^{\perp}$ depends on $N$ too. The noise series is supposed to be infinite as well and the expansions (2.2) and (2.4) are valid for all $\delta$ such that $|\delta|<\delta_{0}=\delta_{0}(N)$.
If $N \rightarrow \infty$, then we come to several problems related to the asymptotic behavior of $\left\|\mathbf{P}_{0}^{\perp}(\delta)-\mathbf{P}_{0}^{\perp}\right\|$. One problem is the behavior of $\delta_{0}(N)$ as $N \rightarrow \infty$ for certain signal and noise series. If $\delta_{0}(N) \rightarrow \infty$, then we can use expansions of $\mathbf{P}_{0}^{\perp}(\delta)$ for any fixed $\delta$ provided that $N$ is sufficiently large. If $\delta_{0}(N)$ is bounded from above (and is separated from zero), then expansions are valid in a certain interval around zero.

Another problem is related to the proximity of $\mathbf{P}_{0}^{\perp}(\delta)$ and $\mathbf{P}_{0}^{\perp}$. In the present paper, we indicate three origins of the proximity $\mathbf{P}_{0}^{\perp}(\delta) \approx \mathbf{P}_{0}^{\perp}$. The first origin can be formulated only in terms of the extremal singular values of the matrices $\mathbf{H}$ and $\mathbf{E}$. Roughly speaking, the difference $\left\|\mathbf{P}_{0}^{\perp}(\delta)-\mathbf{P}_{0}^{\perp}\right\|$ is small if the "signal-to-noise ratio" $\|\mathbf{H}\| /\|\mathbf{E}\|$ is large and the "pseudo-condition number" of the matrix $\mathbf{H} \mathbf{H}^{\mathrm{T}}$, which is defined as the ratio of the maximal and minimal positive eigenvalues of this matrix, is not very large. If these two conditions hold asymptotically, then $\left\|\mathbf{P}_{0}^{\perp}(\delta)-\mathbf{P}_{0}^{\perp}\right\| \rightarrow 0$.

Nevertheless, the convergence $\left\|\mathbf{P}_{0}^{\perp}(\delta)-\mathbf{P}_{0}^{\perp}\right\| \rightarrow 0$ can occur even if the "signal-to-noise ratio" is bounded from above. This happens when both (column and row) pairs of linear spaces produced by matrices $\mathbf{H}$ and $\mathbf{E}$ are asymptotically orthogonal.

The third origin of the convergence is connected with the condition $\mathbf{E E}^{\mathrm{T}} / K \rightarrow \mathbf{I}$, where $\mathbf{I}$ stands for the identity matrix. This case corresponds to the white-noise "noise series". Note that if the "noise series" is random, then the perturbed operator is also random. Moreover, for fixed $\delta$ and $N$ the inequality $|\delta|<\delta_{0}(N)$ holds only with a certain probability.

One more interesting problem related to the convergence $\left\|\mathbf{P}_{0}^{\perp}(\delta)-\mathbf{P}_{0}^{\perp}\right\| \rightarrow 0$ is the form of the main term of the approximation $\mathbf{P}_{0}^{\perp}(\delta) \approx \mathbf{P}_{0}^{\perp}$. Since $\delta$ is fixed and $N \rightarrow \infty$, this term does not necessarily have to be linear in $\delta$.

Now let us explain the style of our results for these asymptotic proximity problems. For this purpose we use simplified but typical examples. More general examples are considered in Section 3.2.

Example 1.1. Let the signal series be $x_{n}=\cos (2 \pi \omega n+\phi)$ with $\omega \in(0,1 / 2)$. Then the pseudo-condition number of the matrix $\mathbf{H H}^{\mathrm{T}}$ is bounded from above as $N \rightarrow \infty$.

a) Suppose that the "noise series" is a linear stationary random series defined on a probability space $(\Omega, \mathcal{F}, \mathbb{P})$. (Exact definitions and restrictions can be found in Lemma 3.2 of Section 3.2.) If $L / N \rightarrow \alpha \in(0,1)$ as $N \rightarrow \infty$, then for a certain $\Omega^{\prime} \in \mathcal{F}$ with $\mathbb{P}\left(\Omega^{\prime}\right)=1$, for any $\omega \in \Omega^{\prime}$, and for any $\delta \in \mathbb{R}$

$$
\begin{aligned}
& \underset{N}{\limsup } \sqrt{N(\ln N)^{-1}}\left\|\mathbf{P}_{0}^{\perp}(\delta)-\mathbf{P}_{0}^{\perp}\right\| \leq b_{1}|\delta| \text { and } \\
& \underset{N}{\limsup } N(\ln N)^{-1}\left\|\mathbf{P}_{0}^{\perp}(\delta)-\mathbf{P}_{0}^{\perp}-\delta \mathbf{V}_{0}^{(1)}\right\| \leq b_{2} \delta^{2},
\end{aligned}
$$

where $\delta \mathbf{V}_{0}^{(1)}$ stands for the linear in $\delta$ term of the difference $\mathbf{P}_{0}^{\perp}(\delta)-\mathbf{P}_{0}^{\perp}$. It should be mentioned that the "signal-to-noise ratio" $\|\mathbf{H}\| /\|\mathbf{E}\|$ tends almost surely to infinity as $N \rightarrow \infty$ in this example. 
b) Assume that the "noise series" is also oscillating and defined by $e_{n}=\cos \left(2 \pi \omega^{\prime} n+\phi^{\prime}\right)$ with $(0,1 / 2) \ni \omega^{\prime} \neq \omega$. If $\min (L, K) \rightarrow \infty$, then for any $\delta \in(-1 / 4,1 / 4)$ and for $N>N_{0}(\delta)$

$$
\begin{aligned}
& \left\|\mathbf{P}_{0}^{\perp}(\delta)-\mathbf{P}_{0}^{\perp}\right\|=|\delta| O(1 / \min (L, K)) \text { and } \\
& \left\|\mathbf{P}_{0}^{\perp}(\delta)-\mathbf{P}_{0}^{\perp}-\mathbf{L}(\delta)\right\|=\delta^{2} O\left(1 / \min \left(L^{2}, K^{2}\right)\right),
\end{aligned}
$$

where $\mathbf{L}(\delta)$ is the non-linear in $\delta$ operator defined in Theorem 2.5. This case corresponds to the asymptotic biorthogonality of matrices $\mathbf{H}$ and $\mathbf{E}$.

c) If white noise stands for the "noise series" and $L=L_{0}=$ const $>d$, then for a certain $\delta_{0}>0$, for some $\Omega^{\prime} \in \mathcal{F}$ with $\mathbb{P}\left(\Omega^{\prime}\right)=1$, for any $|\delta|<\delta_{0}$, and for any $\omega \in \Omega^{\prime}$

$$
\begin{aligned}
& \limsup _{N} \sqrt{N(\ln \ln N)^{-1}}\left\|\mathbf{P}_{0}^{\perp}(\delta)-\mathbf{P}_{0}^{\perp}\right\| \leq c_{1}|\delta| \quad \text { and } \\
& \underset{N}{\lim \sup } N(\ln \ln N)^{-1}\left\|\mathbf{P}_{0}^{\perp}(\delta)-\mathbf{P}_{0}^{\perp}-\mathbf{T}(\delta)\right\| \leq c_{2} \delta^{2},
\end{aligned}
$$

where the non-linear in $\delta$ operator $\mathbf{T}(\delta)$ is defined in Theorem 2.6. The proof in this case is based on the almost sure (briefly, a.s.) convergence $\mathbf{E E}^{\mathrm{T}} / K \rightarrow \mathbf{I}$.

The present paper is organized as follows. Section 2 contains basic theoretical results. General definitions and notation, as well as formulas for the perturbation expansion of the perturbed projector $\mathbf{P}_{0}^{\perp}(\delta)$ are placed in Section 2.1.

In Section 2.2 general upper bounds for the difference $\left\|\mathbf{P}_{0}^{\perp}(\delta)-\mathbf{P}_{0}^{\perp}\right\|$ are constructed and discussed. In Theorem 2.3 we derive the inequality (2.15) such that $\mathbf{P}_{0}^{\perp}(\delta)=\mathbf{P}_{0}^{\perp}$ iff the right-hand side of (2.15) equals zero. (The conditions for the equality of the perturbed and unperturbed projectors are discussed in Section 2.2.1.) Corollaries 2.1 and 2.2 contain interpretable sufficient conditions for the proximity of $\mathbf{P}_{0}^{\perp}(\delta)$ and $\mathbf{P}_{0}^{\perp}$.

The rest of Section 2.2 is devoted to the main terms of the approximation $\mathbf{P}_{0}^{\perp}(\delta) \approx \mathbf{P}_{0}^{\perp}$. These results are collected in Theorems 2.4-2.6 of Section 2.2.3.

In Section 3 we perform the asymptotic analysis of $\left\|\mathbf{P}_{0}^{\perp}(\delta)-\mathbf{P}_{0}^{\perp}\right\|$ in the case when the size of $L \times K$ matrices $\mathbf{H}$ and $\mathbf{E}$ increases. More precisely, we consider conditions for the convergence $\left\|\mathbf{P}_{0}^{\perp}(\delta)-\mathbf{P}_{0}^{\perp}\right\| \rightarrow 0$ as $\max (L, K) \rightarrow \infty$ and study the rate of this convergence.

Two general assertions of this kind are presented in Section 3.1. Starting from Section 3.2, the case of Hankel matrices $\mathbf{H}$ and $\mathbf{E}$ is examined more thoroughly. Several more or less general time series of finite rank are taken as signal and noise series and a number of inequalities similar to those of Example 1.1 are derived. To obtain these inequalities we need to study the asymptotic behavior for the minimal and maximal positive singular values of the corresponding $L \times K$ Hankel matrices as $L+K \rightarrow \infty$. Results for deterministic series are collected in Lemma 3.1.

The case of a stationary random "noise series" is of special interest. Lemma 3.2 presents inequalities for norms of the related Hankel matrices. The proof of Lemma 3.2 is referred to [21, Sect. 7.1 of Appendix 2].

In Section 4 we present several examples of "signal" and "noise" series such that both the exact rates of convergence $\left\|\mathbf{P}_{0}^{\perp}(\delta)-\mathbf{P}_{0}^{\perp}\right\| \rightarrow 0$ and the related main terms are expressed in the explicit form. These examples are rather simple, but they show that refined versions of the inequalities of Section 3.1 can provide precise results.

In general, the term "signal-subspace method" implies that a method provides a small error if the perturbed subspace $\mathbb{U}_{0}^{\perp}(\delta)$ is close to the unperturbed $\mathbb{U}_{0}^{\perp}$. Propositions related to the estimation of LRFs and to the so-called LeastSquare ESPRIT confirm this statement. These propositions are placed in Section 5 .

Singular Spectrum Analysis (briefly, SSA), which can also be considered as a signal-subspace method, shows a different effect. Namely, the SSA reconstruction may not converge to a signal even if $\left\|\mathbf{P}_{0}^{\perp}(\delta)-\mathbf{P}_{0}^{\perp}\right\| \rightarrow 0$ as $N \rightarrow \infty$. The related discussion can be found in Section 5.3.

Proofs of the majority of assertions are placed in Appendix. Some simple but laborsome asymptotic considerations are omitted. Demonstrations of all assertions related to random Hankel matrices are referred to [21].

\section{PERTURBATIONS OF SIGNAL SUBSPACES}

In what follows we do not distinguish linear operators from their matrices. In particular, we use the sign of transposition $\mathbf{B}^{\mathrm{T}}$ instead of the conjugation $\operatorname{sign} \mathbf{B}^{*}$. To avoid misunderstanding, we sometimes use the notation $0_{M}$ for the zero vector in $\mathbb{R}^{M}$ as well as the notation $\mathbf{0}_{M P}$ for the zero operator $\mathbb{R}^{M} \mapsto \mathbb{R}^{P}$.

\subsection{Perturbation series}

Let $\mathbf{H}$ be a real-valued non-zero $\mathbb{R}^{K} \mapsto \mathbb{R}^{L}$ matrix. Then $\mathbf{A} \stackrel{\text { def }}{=} \mathbf{H H}^{\mathrm{T}}$ is a self-adjoint semi-definite operator $\mathbf{A}: \mathbb{R}^{L} \mapsto$ $\mathbb{R}^{L}$. We assume that $d \stackrel{\text { def }}{=} \operatorname{rank} \mathbf{H}<\min (L, K)$. Denote $\Sigma$ the set of eigenvalues of $\mathbf{A}$. Then $0 \in \Sigma \subset[0, \infty)$.

Consider one more real-valued non-zero matrix $\mathbf{E}: \mathbb{R}^{K} \mapsto$ $\mathbb{R}^{L}$ and set $\mathbf{H}(\delta)=\mathbf{H}+\delta \mathbf{E}$. Then

$$
\mathbf{A}(\delta) \stackrel{\text { def }}{=} \mathbf{H}(\delta) \mathbf{H}(\delta)^{\mathrm{T}}=\mathbf{A}+\delta \mathbf{A}^{(1)}+\delta^{2} \mathbf{A}^{(2)}=\mathbf{A}+\mathbf{B}(\delta),
$$

where $\mathbf{A}^{(1)}=\mathbf{H E}^{\mathrm{T}}+\mathbf{E} \mathbf{H}^{\mathrm{T}}, \mathbf{A}^{(2)}=\mathbf{E} \mathbf{E}^{\mathrm{T}}$, and $\mathbf{B}(\delta)=$ $\delta \mathbf{A}^{(1)}+\delta^{2} \mathbf{A}^{(2)}$. Note that both $\mathbf{A}^{(1)}$ and $\mathbf{A}^{(2)}$ are self-adjoint operators, and $\mathbf{A}(\delta)$ is positive semi-definite for any $\delta \in \mathbb{R}$.

We interpret $\mathbf{H}$ as a "signal matrix" and $\mathbf{E}$ as a "noise matrix". It is supposed that we observe the perturbed matrix $\mathbf{H}(\delta)$ and are interested in the "signal subspace" spanned by the columns of the matrix $\mathbf{H}$.

The signal subspace is the orthogonal complement $\mathbb{U}_{0}^{\perp}$ to the eigenspace $\mathbb{U}_{0}$, corresponding to the zero eigenvalue of the matrix $\mathbf{H H}^{\mathrm{T}}$. If $\mathbf{P}_{0}$ stands for the orthogonal projector 
onto $\mathbb{U}_{0}$, then $\mathbf{P}_{0}^{\perp}=\mathbf{I}-\mathbf{P}_{0}$ is the orthogonal projector onto $\mathbb{U}_{0}^{\perp}$, where $\mathbf{I}$ is the identical operator $\mathbb{R}^{L} \mapsto \mathbb{R}^{L}$.

Our goal is to compare the perturbed projector $\mathbf{P}_{0}^{\perp}(\delta)$ with the unperturbed projector $\mathbf{P}_{0}^{\perp}$. In what follows, we set $\mu_{\min }=\min \{\mu \in \Sigma$ such that $\mu>0\}$.

The following assertion can be easily deduced from general results given in [20, Ch. II $\S 3]$. Denote $\mathbf{S}_{0}$ the pseudoinverse to $\mathbf{H} \mathbf{H}^{\mathrm{T}}$. Set $\mathbf{S}_{0}^{(0)}=-\mathbf{P}_{0}$ and $\mathbf{S}_{0}^{(k)}=\mathbf{S}_{0}^{k}$ for $k \geq 1$.

Theorem 2.1. 1. Let $\delta_{0}>0$ and assume that

$$
\|\mathbf{B}(\delta)\|<\mu_{\min } / 2
$$

for all $\delta \in\left(-\delta_{0}, \delta_{0}\right)$. Then the perturbed projector $\mathbf{P}_{0}^{\perp}(\delta)$ admits the expansion

$$
\mathbf{P}_{0}^{\perp}(\delta)=\mathbf{P}_{0}^{\perp}+\sum_{p=1}^{\infty} \mathbf{W}_{p}(\delta),
$$

where

$$
\mathbf{W}_{p}(\delta)=(-1)^{p} \sum_{l_{1}+\ldots+l_{p+1}=p, l_{j} \geq 0} \mathbf{W}_{p}\left(l_{1}, \ldots, l_{p+1}\right)
$$

and

$$
\mathbf{W}_{p}\left(l_{1}, \ldots, l_{p+1}\right)=\mathbf{S}_{0}^{\left(l_{1}\right)} \mathbf{B}(\delta) \mathbf{S}_{0}^{\left(l_{2}\right)} \ldots \mathbf{S}_{0}^{\left(l_{p}\right)} \mathbf{B}(\delta) \mathbf{S}_{0}^{\left(l_{p+1}\right)} .
$$

In addition,

$$
\mathbf{P}_{0}^{\perp}(\delta)=\mathbf{P}_{0}^{\perp}+\sum_{n=1}^{\infty} \delta^{n} \mathbf{V}_{0}^{(n)}
$$

where

$$
\mathbf{V}_{0}^{(n)}=\sum_{p=\lceil n / 2\rceil}^{n}(-1)^{p} \sum_{\begin{array}{c}
s_{1}+\cdots+s_{p}=n, s_{i}=1,2 \\
l_{1}+\cdots+l_{p+1}=p, l_{j} \geq 0
\end{array}} \mathbf{V}_{0}^{(n)}(\mathbf{s}, \ell),
$$

$\mathbf{s}=\left(s_{1}, \ldots, s_{p}\right), \ell=\left(l_{1}, \ldots, l_{p+1}\right)$, and

$$
\mathbf{V}_{0}^{(n)}(\mathbf{s}, \ell)=\mathbf{S}_{0}^{\left(l_{1}\right)} \mathbf{A}^{\left(s_{1}\right)} \mathbf{S}_{0}^{\left(l_{2}\right)} \ldots \mathbf{A}^{\left(s_{p}\right)} \mathbf{S}_{0}^{\left(l_{p+1}\right)} .
$$

Remark 2.1. 1. Both series (2.2) and (2.4) converge in the spectral norm.

2. Denote

$$
B(\delta)=|\delta|\left\|\mathbf{A}^{(1)}\right\|+\delta^{2}\left\|\mathbf{A}^{(2)}\right\| .
$$

If $\delta_{0}>0$ and $B\left(\delta_{0}\right)=\mu_{\min } / 2$, then the inequality (2.1) is valid for all $\delta$ such that $|\delta|<\delta_{0}$.

3. Since $\mathbf{H H}^{\mathrm{T}}=\sum_{\mu>0} \mu \mathbf{P}_{\mu}$, then $\mathbf{S}_{0}=\sum_{\mu>0} \mathbf{P}_{\mu} / \mu$. (Here and further we write $\sum_{\mu>0}$ instead of $\sum_{\Sigma \ni \mu>0}$.) It is easy to show that $\left\|\mathbf{S}_{0}^{(k)}\right\|=1 / \mu_{\min }^{k}$ for any $k \geq 0$.

4. The coefficient $\mathbf{V}_{0}^{(1)}$ of the linear in $\delta$ term of the righthand side of (2.4) has the form

$$
\begin{aligned}
\mathbf{V}_{0}^{(1)} & =\mathbf{P}_{0} \mathbf{A}^{(1)} \mathbf{S}_{0}+\mathbf{S}_{0} \mathbf{A}^{(1)} \mathbf{P}_{0} \\
& =\mathbf{P}_{0} \mathbf{E} \mathbf{H}^{\mathrm{T}} \mathbf{S}_{0}+\mathbf{S}_{0} \mathbf{H} \mathbf{E}^{\mathrm{T}} \mathbf{P}_{0}
\end{aligned}
$$

5. Accurate calculations show that

$$
\begin{aligned}
\mathbf{V}_{0}^{(2)}= & \mathbf{P}_{0} \mathbf{A}^{(2)} \mathbf{S}_{0}+\mathbf{S}_{0} \mathbf{A}^{(2)} \mathbf{P}_{0} \\
& +\mathbf{P}_{0} \mathbf{A}^{(1)} \mathbf{P}_{0} \mathbf{A}^{(1)} \mathbf{S}_{0}^{2}+\mathbf{P}_{0} \mathbf{A}^{(1)} \mathbf{S}_{0}^{2} \mathbf{A}^{(1)} \mathbf{P}_{0} \\
& +\mathbf{S}_{0}^{2} \mathbf{A}^{(1)} \mathbf{P}_{0} \mathbf{A}^{(1)} \mathbf{P}_{0}-\mathbf{P}_{0} \mathbf{A}^{(1)} \mathbf{S}_{0} \mathbf{A}^{(1)} \mathbf{S}_{0} \\
& -\mathbf{S}_{0} \mathbf{A}^{(1)} \mathbf{P}_{0} \mathbf{A}^{(1)} \mathbf{S}_{0}-\mathbf{S}_{0} \mathbf{A}^{(1)} \mathbf{S}_{0} \mathbf{A}^{(1)} \mathbf{P}_{0}
\end{aligned}
$$

6 . The term $\mathbf{W}_{1}(\delta)$ in the right-hand side of $(2.2)$ can be expressed as

$$
\begin{aligned}
\mathbf{W}_{1}(\delta) & =\mathbf{P}_{0} \mathbf{B}(\delta) \mathbf{S}_{0}+\mathbf{S}_{0} \mathbf{B}(\delta) \mathbf{P}_{0} \\
& =\delta \mathbf{V}_{0}^{(1)}+\delta^{2}\left(\mathbf{P}_{0} \mathbf{A}^{(2)} \mathbf{S}_{0}+\mathbf{S}_{0} \mathbf{A}^{(2)} \mathbf{P}_{0}\right) .
\end{aligned}
$$

\subsection{Approximation errors}

In this section we derive upper bounds for the norm $\left\|\mathbf{P}_{0}^{\perp}(\delta)-\mathbf{P}_{0}^{\perp}\right\|$. These bounds produce interpretable sufficient conditions for the proximity of linear spaces $\mathbb{U}_{0}^{\perp}(\delta)$ and $\mathbb{U}_{0}^{\perp}$. We also present operators that can play a role of the main terms of the difference $\mathbf{P}_{0}^{\perp}(\delta)-\mathbf{P}_{0}^{\perp}$.

\subsubsection{Zero perturbation effects}

We start with the necessary and sufficient conditions for the equality $\mathbf{P}_{0}^{\perp}(\delta)=\mathbf{P}_{0}^{\perp}$. Consider the function $B(\delta)$ defined in (2.6).

Theorem 2.2. Let $\delta_{0}>0$ and assume that $B\left(\delta_{0}\right)=\mu_{\min } / 2$. Then the following assertions are equivalent:

$$
\text { 1. } \mathbf{P}_{0}^{\perp}(\delta)=\mathbf{P}_{0}^{\perp} \text { for all } \delta \in\left(-\delta_{0}, \delta_{0}\right) \text {; }
$$

2.

$$
\mathbf{S}_{0} \mathbf{H E}^{\mathrm{T}} \mathbf{P}_{0}=\mathbf{S}_{0} \mathbf{E E}^{\mathrm{T}} \mathbf{P}_{0}=\mathbf{0}_{L L}
$$

$$
\mathbf{S}_{0} \mathbf{B}(\delta) \mathbf{P}_{0}=\mathbf{0}_{L L}
$$

for all $\delta$ from a certain neighbourhood of zero;

$$
4 .
$$

$$
\begin{aligned}
& \mathbf{S}_{0} \mathbf{H E}^{\mathrm{T}} \mathbf{P}_{0}+\mathbf{P}_{0} \mathbf{H} \mathbf{E}^{\mathrm{T}} \mathbf{S}_{0} \\
& =\mathbf{S}_{0} \mathbf{E} \mathbf{E}^{\mathrm{T}} \mathbf{P}_{0}+\mathbf{P}_{0} \mathbf{E} \mathbf{E}^{\mathrm{T}} \mathbf{S}_{0}=\mathbf{0}_{L L}
\end{aligned}
$$

5.

$$
\mathbf{H E}^{\mathrm{T}} \mathbf{P}_{0}=\mathbf{0}_{L L} \text { and } \mathbf{H}^{\mathrm{T}} \mathbf{E} \mathbf{E}^{\mathrm{T}} \mathbf{P}_{0}=\mathbf{0}_{L K} .
$$

Remark 2.2. 1. The equality $\mathbf{S}_{0} \mathbf{E E}^{\mathrm{T}} \mathbf{P}_{0}=\mathbf{0}_{L L}$ is equivalent to $\mathbf{P}_{0}^{\perp} \mathbf{E E}^{\mathrm{T}} \mathbf{P}_{0}=\mathbf{0}_{L L}$.

2. Let us discuss the conditions (2.13).

Let $\mathbb{U}_{E}$ stand for the linear space spanned by the columns of the matrix $\mathbf{E}$ and denote $s=\operatorname{dim} \mathbb{U}_{E}$. Suppose that there exists an orthonormal basis $P_{1}, \ldots, P_{s}$ of the space $\mathbb{U}_{E}$ such that a) each $P_{i}$ is an eigenvector of the matrix $\mathbf{E E}^{\mathrm{T}}$, b) $P_{1}, \ldots, P_{l} \in \mathbb{U}_{0}^{\perp}$, and c) $P_{l+1}, \ldots, P_{s} \in \mathbb{U}_{0}$ for 
some $0 \leq l \leq s$. Then $\mathbf{H}^{\mathrm{T}} \mathbf{E} \mathbf{E}^{\mathrm{T}} \mathbf{P}_{0}=\mathbf{0}_{L K}$. Note that the latter equality is equivalent to $\mathbf{P}_{0}^{\perp} \mathbf{E} \mathbf{E}^{\mathrm{T}} \mathbf{P}_{0}=\mathbf{0}_{L L}$.

Indeed, consider the Singular Value Decomposition $\mathbf{E E}^{\mathrm{T}}=\sum_{i=1}^{s} \nu_{i} P_{i} P_{i}^{\mathrm{T}}$ of the matrix $\mathbf{E E}^{\mathrm{T}}$. Then $\mathbf{P}_{0} P_{i}=0_{L}$ for $1 \leq i \leq l, \mathbf{P}_{0}^{\perp} P_{i}=0_{L}$ for $i>l$, and

$$
\begin{aligned}
\mathbf{P}_{0}^{\perp} \mathbf{E E}^{\mathrm{T}} \mathbf{P}_{0}= & \sum_{i=1}^{l} \nu_{i} \mathbf{P}_{0}^{\perp} P_{i}\left(P_{i}^{\mathrm{T}} \mathbf{P}_{0}\right) \\
& +\sum_{i=l+1}^{s} \nu_{i}\left(\mathbf{P}_{0}^{\perp} P_{i}\right) P_{i}^{\mathrm{T}} \mathbf{P}_{0}=\mathbf{0}_{L L} .
\end{aligned}
$$

There are several important particular cases related to the situation under discussion.

If $l=s$, then $\mathbb{U}_{E} \subset \mathbb{U}_{0}^{\perp}, \mathbf{E}^{\mathrm{T}} \mathbf{P}_{0}=\mathbf{0}_{L K}$, and both conditions of (2.13) are fulfilled. The example when $\mathbf{E}$ is proportional to $\mathbf{H}$ (then the equality $\mathbf{P}_{0}^{\perp}(\delta)=\mathbf{P}_{0}^{\perp}$ becomes evident) is just a particular case of the inclusion $\mathbb{U}_{E} \subset \mathbb{U}_{0}^{\perp}$.

If $l<s$, then the natural sufficient condition for the equality $\mathbf{H} \mathbf{E}^{\mathrm{T}} \mathbf{P}_{0}=\mathbf{0}_{L L}$ is $\mathbf{H} \mathbf{E}^{\mathrm{T}}=\mathbf{0}_{L L}$. The analogous sufficient condition $\mathbf{H}^{\mathrm{T}} \mathbf{E}=\mathbf{0}_{K K}$ for the equality $\mathbf{H}^{\mathrm{T}} \mathbf{E} \mathbf{E}^{\mathrm{T}} \mathbf{P}_{0}=$ $\mathbf{0}_{L K}$ corresponds to the case $l=0$ with $\mathbb{U}_{E} \subset \mathbb{U}_{0}$.

Lastly, suppose that $s=L$ and that all singular values of the matrix $\mathbf{E}$ coincide. If we take $l=d$ and define $P_{1}, \ldots, P_{d}$ as an orthonormal basis of $\mathbb{U}_{0}^{\perp}$, then we get the second equality of (2.13). This case corresponds to a special "noise matrix" $\mathbf{E}$ with $\mathbf{E E}^{\mathrm{T}}$ proportional to $\mathbf{I}$.

Consider more explicitly the case where both $\mathbf{H E}^{\mathrm{T}}$ and $\mathbf{H}^{\mathrm{T}} \mathbf{E}$ are zero matrices. Then $\mathbf{P}_{0}^{\perp}(\delta)=\mathbf{P}_{0}^{\perp}$ under the conditions of Theorem 2.2.

We call matrices $\mathbf{H}$ and $\mathbf{E}$ right-orthogonal if $\mathbf{H E}^{\mathrm{T}}=$ $\mathbf{0}_{L L}$, or, equivalently, $\mathbf{E} \mathbf{H}^{\mathrm{T}}=\mathbf{0}_{L L}$. If $\mathbf{H E}^{\mathrm{T}}=\mathbf{0}_{L L}$ and $\mathbf{H}^{\mathrm{T}} \mathbf{E}=\mathbf{0}_{K K}$ (the latter equality means that $\mathbf{H}$ and $\mathbf{E}$ are left-orthogonal), then the matrices are called biorthogonal. The biorthogonality condition corresponds to the notion of weak separability in SSA (see [13, Sections 1.5 and 6.1]).

The following statement elucidates the notion of left orthogonality.

Lemma 2.1. Let $\mathbf{H}$ and $\mathbf{E}$ be non-zero $L \times K$ matrices.

1. If $\mathbf{H}$ and $\mathbf{E}$ are left-orthogonal, then $0 \in \Sigma, \mathbf{P}_{0} \mathbf{E}=\mathbf{E}$, and $\mathbf{P}_{\mu} \mathbf{E}=\mathbf{0}_{K L}$ for all non-zero $\mu \in \Sigma$.

2. If $\mathbf{P}_{\mu} \mathbf{E}=\mathbf{0}_{K L}$ for all non-zero $\mu \in \Sigma$, then $\mathbf{H}$ and $\mathbf{E}$ are left-orthogonal.

Remark 2.3. Denote $\Sigma_{\mathbf{H}}$ and $\Sigma_{\mathbf{E}}(\delta)$ the sets of positive eigenvalues of the operators $\mathbf{A}=\mathbf{H} \mathbf{H}^{\mathrm{T}}$ and $\delta^{2} \mathbf{E} \mathbf{E}^{\mathrm{T}}=$ $\delta^{2} \mathbf{A}^{(2)}$, respectively.

Suppose that $\mathbf{H}$ and $\mathbf{E}$ are biorthogonal and assume additionally that $\Sigma_{\mathbf{H}} \cap \Sigma_{\mathbf{E}}(\delta)=\varnothing$. (Note that this corresponds to the strong separability in SSA, see $[13, \S 1.5]$.) Then it is easy to see that SVD of the matrix $\mathbf{H}+\delta \mathbf{E}$ is the sum of SVDs of the matrices $\mathbf{H}$ and $\delta \mathbf{E}$. Consequently, both singular values and the related singular vectors of the operator $\mathbf{H}$ do not change under the perturbation $\mathbf{H} \mapsto \mathbf{H}+\delta \mathbf{E}$.
Imposing also the condition $\delta_{0}^{2}\left\|\mathbf{E E}^{\mathrm{T}}\right\|=\mu_{\text {min }}$, we obtain that $\mathbf{P}_{0}^{\perp}(\delta)=\mathbf{P}_{0}^{\perp}$ for all $\delta \in\left(\delta_{0}, \delta_{0}\right)$. This condition tells us that each positive eigenvalue of the matrix $\mathbf{H H}^{\mathrm{T}}$ is greater than all eigenvalues of the matrix $\delta^{2} \mathbf{E} \mathbf{E}^{\mathrm{T}}$.

This means that in the case of biorthogonality, we have $\mathbf{P}_{0}^{\perp}(\delta)=\mathbf{P}_{0}^{\perp}$ for all $\delta \in\left(\delta_{0}, \delta_{0}\right)$ under the necessary and sufficient condition $\delta_{0}^{2}\left\|\mathbf{E E}^{\mathrm{T}}\right\|=\mu_{\text {min }}$ rather than under the sufficient condition $\delta_{0}^{2}\left\|\mathbf{E E}^{\mathrm{T}}\right\|=\mu_{\min } / 2$ of Theorem 2.2.

If the matrices $\mathbf{H}$ and $\mathbf{E}$ are right-orthogonal, then $\mathbf{B}(\delta)=\delta^{2} \mathbf{A}^{(2)}$, the condition $\delta^{2}\left\|\mathbf{E} \mathbf{E}^{\mathrm{T}}\right\|<\mu_{\min } / 2$ provides the validity of Theorem 2.1, and, due to (2.2), the expansion of the perturbed projector takes the form

$$
\begin{aligned}
& \mathbf{P}_{0}^{\perp}(\delta)=\mathbf{P}_{0}^{\perp} \\
& +\sum_{p=1}^{\infty}(-1)^{p} \delta^{2 p} \sum_{\substack{l_{1}+\ldots+l_{p+1}=p \\
l_{j} \geq 0}} \mathbf{S}_{0}^{\left(l_{1}\right)} \mathbf{A}^{(2)} \ldots \mathbf{A}^{(2)} \mathbf{S}_{0}^{\left(l_{p+1}\right)} .
\end{aligned}
$$

\subsubsection{General upper bounds}

Roughly speaking, Theorem 2.2 shows that under the conditions $B\left(\delta_{0}\right)=\mu_{\min } / 2$ and $|\delta|<\delta_{0}$, the equalities $\mathbf{S}_{0} \mathbf{B}(\delta) \mathbf{P}_{0}=\mathbf{0}$ and $\mathbf{P}_{0}^{\perp}(\delta)=\mathbf{P}_{0}^{\perp}$ are equivalent. Moreover,

$$
\left\|\mathbf{S}_{0} \mathbf{B}(\delta) \mathbf{P}_{0}\right\| \leq \frac{\|\mathbf{B}(\delta)\|}{\mu_{\min }} \leq \frac{B(\delta)}{\mu_{\min }} \leq 1 / 2 .
$$

These considerations give rise to the supposition that $\left\|\mathbf{S}_{0} \mathbf{B}(\delta) \mathbf{P}_{0}\right\|$ can serve as a natural measure of the proximity $\mathbf{P}_{0}^{\perp}(\delta) \approx \mathbf{P}_{0}^{\perp}$.

Theorem 2.3. If $\delta_{0}>0$ and $\|\mathbf{B}(\delta)\| / \mu_{\min }<1 / 4$ for all $\delta \in\left(-\delta_{0}, \delta_{0}\right)$, then

$$
\left\|\mathbf{P}_{0}^{\perp}(\delta)-\mathbf{P}_{0}^{\perp}\right\| \leq 4 C \frac{\left\|\mathbf{S}_{0} \mathbf{B}(\delta) \mathbf{P}_{0}\right\|}{1-4\|\mathbf{B}(\delta)\| / \mu_{\min }},
$$

where $C=e^{1 / 6} / \sqrt{\pi}$.

Let us discuss the conditions which guarantee that the right-hand side of (2.15) is small.

The case of big signal and small noise matrices.

We start with a condition formulated in terms of eigenvalues of matrices $\mathbf{A}=\mathbf{H} \mathbf{H}^{\mathrm{T}}$ and $\mathbf{A}^{(2)}=\mathbf{E} \mathbf{E}^{\mathrm{T}}$. Denote

$$
\Theta_{1}=\sqrt{\frac{\nu_{\max }}{\mu_{\max }}} \text { and } \Theta_{2}=\frac{\mu_{\max }}{\mu_{\min }},
$$

where $\nu_{\max }=\left\|\mathbf{A}^{(2)}\right\|$. Note that $\Theta_{1}$ is a sort of the "noiseto-signal ratio". Since $\Theta_{2}=\|\mathbf{A}\|\left\|\mathbf{S}_{0}\right\|$ and since $\mathbf{S}_{0}$ is the pseudo-inverse to $\mathbf{A}$, then $\Theta_{2}$ can be called as the "pseudocondition number" of the matrix $\mathbf{A}$.

Corollary 2.1. Under the conditions of Theorem 2.3,

$$
\left\|\mathbf{P}_{0}^{\perp}(\delta)-\mathbf{P}_{0}^{\perp}\right\| \leq 4 C \frac{\|\mathbf{B}(\delta)\|}{\mu_{\min }} \frac{1}{1-4\|\mathbf{B}(\delta)\| / \mu_{\min }}
$$

Perturbation expansions of signal subspaces 301 
with

$$
\frac{\|\mathbf{B}(\delta)\|}{\mu_{\min }} \leq \frac{B(\delta)}{\mu_{\min }} \leq 2|\delta| \Theta_{1} \Theta_{2}+\delta^{2} \Theta_{1}^{2} \Theta_{2} .
$$

Remark 2.4. 1. The inequality (2.16) shows that the difference $\mathbf{P}_{0}^{\perp}(\delta)-\mathbf{P}_{0}^{\perp}$ is small if the norm of the perturbation operator $\mathbf{B}(\delta)$ is substantially smaller than the minimal positive eigenvalue of the matrix $\mathbf{H H}^{\mathrm{T}}$.

2. Inequalities (2.16), (2.17) jointly yield sufficient conditions for close proximity of the projectors $\mathbf{P}_{0}^{\perp}(\delta)$ and $\mathbf{P}_{0}^{\perp}$ in terms of the eigenvalues of the matrices $\mathbf{H} \mathbf{H}^{\mathrm{T}}$ and $\mathbf{E} \mathbf{E}^{\mathrm{T}}$. Roughly speaking, for fixed $\delta$ the difference $\mathbf{P}_{0}^{\perp}(\delta)-\mathbf{P}_{0}^{\perp}$ is small if $\Theta_{1} \ll 1$ (this means that the signal matrix $\mathbf{H}$ is "big" and the noise matrix $\mathbf{E}$ is "small") and if the positive spectrum of the matrix $\mathbf{H H}^{\mathrm{T}}$ is not wide-spread in the sense that the quotient $\Theta_{2}$ is not very large.

In particular, if $2|\delta| \Theta_{1} \Theta_{2}+\delta^{2} \Theta_{1}^{2} \Theta_{2} \leq \varepsilon<1 / 4$, then

$$
\left\|\mathbf{P}_{0}^{\perp}(\delta)-\mathbf{P}_{0}^{\perp}\right\| \leq 4 C \frac{\varepsilon}{1-4 \varepsilon} .
$$

The case of the approximate orthogonalities.

The upper bound in the inequalities (2.16), (2.17) is rather rough, since it does not incorporate the orthogonality properties of the matrices $\mathbf{H}$ and $\mathbf{E}$. (On the other hand, this upper bound shows that $\mathbf{P}_{0}^{\perp}(\delta)$ can be close to $\mathbf{P}_{0}^{\perp}$ without any orthogonalities.) Even if these matrices are biorthogonal, still the right-hand side of (2.16) remains positive: in this case (2.16) takes a form

$$
\left\|\mathbf{P}_{0}^{\perp}(\delta)-\mathbf{P}_{0}^{\perp}\right\| \leq 4 C \delta^{2} \frac{\nu_{\max }}{\mu_{\min }} \frac{1}{1-4 \delta^{2} \nu_{\max } / \mu_{\min }} .
$$

To improve this inconvenience we start with the following assertion concerning the minimal principal angle between linear spaces.

Proposition 2.1. Consider matrices $\mathbf{M}_{1}, \mathbf{M}_{2}$ acting from $\mathbb{R}^{K}$ onto $\mathbb{R}^{L}$. Denote $\theta_{\text {min }}$ the minimal principal angle between subspaces $\mathbb{U}_{1}$ and $\mathbb{U}_{2}$, that are spanned by the columns of matrices $\mathbf{M}_{1}$ and $\mathbf{M}_{2}$. Lastly, let $\sigma_{1}^{(\min )}, \sigma_{2}^{(\min )}$ stand for the minimal singular values of $\mathbf{M}_{1}, \mathbf{M}_{2}$, respectively. Then

$$
\begin{aligned}
& \sigma_{1}^{(\min )} \sigma_{2}^{(\min )} \cos \left(\theta_{\min }\right) \\
& \leq\left\|\mathbf{M}_{1}^{\mathrm{T}} \mathbf{M}_{2}\right\| \leq\left\|\mathbf{M}_{1}\right\|\left\|\mathbf{M}_{2}\right\| \cos \left(\theta_{\min }\right) .
\end{aligned}
$$

The following assertion follows from Theorem 2.3 and Proposition 2.1. Denote $\theta_{r}\left(\theta_{l}\right)$ minimal principal angles between subspaces spanned by rows (columns) of matrices $\mathbf{H}$ and $\mathbf{E}$.

Corollary 2.2. Under the conditions of Theorem 2.3,

$$
\left\|\mathbf{P}_{0}^{\perp}(\delta)-\mathbf{P}_{0}^{\perp}\right\| \leq 4 C \frac{\left\|\mathbf{S}_{0} \mathbf{B}(\delta)\right\|}{1-4\|\mathbf{B}(\delta)\| / \mu_{\min }}
$$

and

$$
\left\|\mathbf{S}_{0} \mathbf{B}(\delta)\right\| \leq|\delta| \Theta_{1} \Theta_{2}\left(2 \cos \left(\theta_{r}\right)+|\delta| \Theta_{1} \cos \left(\theta_{l}\right)\right) .
$$

Remark 2.5. 1. Both $\cos \left(\theta_{r}\right)$ and $\cos \left(\theta_{l}\right)$ serve as proper measures of right and left orthogonalities for the matrices $\mathbf{H}$ and $\mathbf{E}$.

2. Using the inequalities (2.20) and (2.21) we obtain sufficient conditions for the proximity $\mathbf{P}_{0}^{\perp}(\delta) \approx \mathbf{P}_{0}^{\perp}$ in the case when the "noise-to-signal ratio" $\Theta_{1}$ is not small. Thus, the "pseudo-condition number" $\Theta_{2}$ of the matrix $\mathbf{H H}^{\mathrm{T}}$ should not be very large and both pairs of linear spaces produced by the matrices $\mathbf{H}$ and $\mathbf{E}$ should be almost orthogonal.

\subsubsection{Main terms of the approximations}

In this section we discuss special refinements of the inequalities (2.15), (2.16), and (2.20). More precisely, we present operators that can be considered as the main terms of the difference $\mathbf{P}_{0}^{\perp}(\delta)-\mathbf{P}_{0}^{\perp}$ in the case when this difference is small by norm. The results show that the inequalities (2.15), (2.16), and (2.20) produce different (though connected with each other) versions of the main terms.

The case of big signal and small noise matrices.

Let us start with the inequality (2.16).

Theorem 2.4. Under the conditions of Theorem 2.3,

$$
\begin{aligned}
& \left\|\mathbf{P}_{0}^{\perp}(\delta)-\mathbf{P}_{0}^{\perp}-\mathbf{W}_{1}(\delta)\right\| \\
& \quad \leq 16 C\left(\frac{\|\mathbf{B}(\delta)\|}{\mu_{\min }}\right)^{2} \frac{1}{1-4\|\mathbf{B}(\delta)\| / \mu_{\min }},
\end{aligned}
$$

where $\mathbf{W}_{1}(\delta)$ is given by (2.9) and $C=e^{1 / 6} / \sqrt{\pi}$.

Let us compare (2.22) and (2.16) with the help of the inequality (2.17). If $\mathbf{B}(\delta) / \mu_{\min }$ is small, then the inequality (2.22) shows that the operator $\mathbf{W}_{1}(\delta)$ can be considered as the main term of the difference $\mathbf{P}_{0}^{\perp}(\delta)-\mathbf{P}_{0}^{\perp}$. In particular, $g\left\|\mathbf{P}_{0}^{\perp}(\delta)-\mathbf{P}_{0}^{\perp}-\mathbf{W}_{1}(\delta)\right\| \ll\left\|\mathbf{P}_{0}^{\perp}(\delta)-\mathbf{P}_{0}^{\perp}\right\|$ if the "noise-to-signal ratio" $\Theta_{1}$ is small and the "pseudocondition number" $\Theta_{2}$ is not very large.

For example, if $2|\delta| \Theta_{1} \Theta_{2}+\delta^{2} \Theta_{1}^{2} \Theta_{2} \leq \varepsilon<1 / 4$, then

$$
\left\|\mathbf{P}_{0}^{\perp}(\delta)-\mathbf{P}_{0}^{\perp}-\mathbf{W}_{1}(\delta)\right\| \leq 16 C \frac{\varepsilon^{2}}{1-4 \varepsilon},
$$

while $\left\|\mathbf{P}_{0}^{\perp}(\delta)-\mathbf{P}_{0}^{\perp}\right\|$ satisfies (2.18).

The case of approximate orthogonalities.

Let us turn to the upper bound (2.20).

We start with a simple remark. Denote $\mathbf{A}_{0}^{(2)}=\mathbf{P}_{0} \mathbf{A}^{(2)} \mathbf{P}_{0}$ and assume that $B\left(\delta_{0}\right)<\mu_{\min }$. Then it is easy to check that the operator $\mathbf{I}-\delta^{2} \mathbf{A}_{0}^{(2)} / \mu$ is invertible for any positive $\mu \in \Sigma$ and for any $\delta \in\left(-\delta_{0}, \delta_{0}\right)$ since

$$
\delta^{2} \frac{\left\|\mathbf{A}_{0}^{(2)}\right\|}{\mu} \leq \delta_{0}^{2} \frac{\nu_{\max }}{\mu_{\min }} \leq \frac{B\left(\delta_{0}\right)}{\mu_{\min }}<1 .
$$

Theorem 2.5. Assume that $\delta_{0}>0, B\left(\delta_{0}\right)=\mu_{\min } / 4$ and $|\delta|<\delta_{0}$. Denote

302 V. Nekrutkin

$$
\mathbf{L}_{1}(\delta)=\sum_{\mu>0} \frac{\mathbf{P}_{\mu} \mathbf{B}(\delta) \mathbf{P}_{0}}{\mu}\left(\mathbf{I}-\delta^{2} \mathbf{A}_{0}^{(2)} / \mu\right)^{-1}
$$


and $\mathbf{L}(\delta)=\mathbf{L}_{1}(\delta)+\mathbf{L}_{1}^{\mathrm{T}}(\delta)$. Then

$$
\left\|\mathbf{P}_{0}^{\perp}(\delta)-\mathbf{P}_{0}^{\perp}-\mathbf{L}(\delta)\right\| \leq 16 C \frac{\left\|\mathbf{S}_{0} \mathbf{B}(\delta)\right\|\left\|\mathbf{S}_{0} \mathbf{B}(\delta) \mathbf{P}_{0}\right\|}{1-4\|\mathbf{B}(\delta)\| / \mu_{\min }}
$$

where $C=e^{1 / 6} / \sqrt{\pi}$.

The operator $\mathbf{L}(\delta)$ admits another representation. Denote

$$
\mathbf{K}_{1}(\delta)=\sum_{\mu>0} \frac{\mathbf{P}_{\mu} \mathbf{B}(\delta) \mathbf{A}_{0}^{(2)}}{\mu^{2}}\left(\mathbf{I}-\delta^{2} \mathbf{A}_{0}^{(2)} / \mu\right)^{-1}
$$

and put $\mathbf{K}(\delta)=\mathbf{K}_{1}(\delta)+\mathbf{K}_{1}^{\mathrm{T}}(\delta)$.

Proposition 2.2. Under the conditions and notation of Theorem 2.5,

$$
\mathbf{L}(\delta)=\mathbf{W}_{1}(\delta)+\delta^{2} \mathbf{K}(\delta),
$$

where $\mathbf{W}_{1}(\delta)$ is defined in (2.9).

Let us discuss the result of Theorem 2.5. The upper bound (2.20) is reasonable when $\|\mathbf{B}(\delta)\| / \mu_{\min }$ is not small and $\left\|\mathbf{S}_{0} \mathbf{B}(\delta)\right\|$ is small enough.

Assume that $\left\|\mathbf{S}_{0} \mathbf{B}(\delta)\right\| \approx \varepsilon$. Since

$$
\left\|\mathbf{S}_{0} \mathbf{B}(\delta) \mathbf{P}_{0}\right\| \leq\left\|\mathbf{S}_{0} \mathbf{B}(\delta)\right\|
$$

then the right-hand side of (2.24) is proportional to $\varepsilon^{2}$ while the right-hand side of (2.20) is proportional to $\varepsilon$. This means that the operator $\mathbf{L}(\delta)$ can be considered as the main term of the difference $\mathbf{P}_{0}^{\perp}(\delta)-\mathbf{P}_{0}^{\perp}$.

General case.

Let us study the general upper bound given in Theorem 2.3. It follows from the discussion of Section 2.2.1 that the inequality (2.15) generally gives weaker conditions for the proximity $\mathbf{P}_{0}^{\perp}(\delta) \approx \mathbf{P}_{0}^{\perp}$ than the inequalities (2.16) and (2.20).

Let us present a version of the main term of the difference $\mathbf{P}_{0}^{\perp}(\delta)-\mathbf{P}_{0}^{\perp}$ in this general case.

Theorem 2.6. Under the conditions of Theorem 2.5,

$$
\left\|\mathbf{P}_{0}^{\perp}(\delta)-\mathbf{P}_{0}^{\perp}-\mathbf{T}(\delta)\right\| \leq 16 C \frac{\left\|\mathbf{S}_{0} \mathbf{B}(\delta) \mathbf{P}_{0}\right\|^{2}}{1-4\|\mathbf{B}(\delta)\| / \mu_{\min }},
$$

where $C=e^{1 / 6} / \sqrt{\pi}, \mathbf{T}(\delta)=\mathbf{T}_{1}(\delta)+\mathbf{T}_{1}^{\mathrm{T}}(\delta)$,

$$
\mathbf{T}_{1}(\delta)=\sum_{i=0}^{\infty}(-1)^{i} \sum_{\mu_{1}, \ldots, \mu_{i}>0} \mathbf{J}_{i} \mathbf{L}_{1}(\delta) \mathbf{G}_{i},
$$

$\mathbf{J}_{i}=\mathbf{J}_{i}\left(\mu_{1}, \ldots, \mu_{i}\right)=\prod_{k=1}^{i}\left(\mathbf{P}_{\mu_{k}} \mathbf{B}(\delta) / \mu_{k}\right)$, and

$$
\mathbf{G}_{i}=\mathbf{G}_{i}\left(\mu_{1}, \ldots, \mu_{i}\right)=\prod_{k=1}^{i}\left(\mathbf{I}-\delta^{2} \mathbf{A}_{0}^{(2)} / \mu_{k}\right)^{-1} .
$$

\section{SUBSPACE PERTURBATIONS FOR HANKEL MATRICES OF LARGE SIZE}

Let $\mathrm{F}=\left(x_{0}, \ldots, x_{N-1}, \ldots\right)$ and $\mathrm{E}=\left(e_{0}, \ldots, e_{N-1}, \ldots\right)$. We treat $\mathrm{F}$ as a "signal series" and $\mathrm{E}$ as a "noise series". For fixed $N$ we choose the window length $L$ and construct two Hankel ("trajectory") matrices

$$
\mathbf{H}=\mathbf{H}_{K, L}=\left(\begin{array}{ccccc}
x_{0} & x_{1} & \ldots & x_{K-2} & x_{K-1} \\
\vdots & \vdots & \ddots & \vdots & \vdots \\
x_{L-1} & x_{L} & \ldots & x_{N-2} & x_{N-1}
\end{array}\right)
$$

and

$$
\mathbf{E}=\mathbf{E}_{K, L}=\left(\begin{array}{ccccc}
e_{0} & e_{1} & \ldots & e_{K-2} & e_{K-1} \\
\vdots & \vdots & \ddots & \vdots & \vdots \\
e_{L-1} & e_{L} & \ldots & e_{N-2} & e_{N-1}
\end{array}\right)
$$

where $K=N-L+1$. In terms of Section 2, $\mathbf{H}$ serves as a signal matrix and $\mathbf{E}$ is a noise matrix.

It is clear that $\operatorname{rank} \mathbf{H} \leq \min (L, K)$. As it was already mentioned, we are interested in the case $\operatorname{rank} \mathbf{H}<$ $\min (L, K)$. To provide this condition, we assume that $\mathrm{F}$ is governed by a minimal Linear Recurrent Formula of order $d$. Then $\operatorname{rank} \mathbf{H}=d$ for all $L$ and $K$ such that $\min (L, K) \geq d$.

Consider the perturbed series $\mathrm{F}(\delta)=\mathrm{F}+\delta \mathrm{E}$ and construct the corresponding Hankel matrix $\mathbf{H}(\delta)=\mathbf{H}+\delta \mathbf{E}$. Then we can apply all notation and results of Section 2 to this particular case of matrices $\mathbf{H}$ and $\mathbf{E}$.

In the present section we derive conditions providing the convergence $\left\|\mathbf{P}_{0}^{\perp}(\delta)-\mathbf{P}_{0}^{\perp}\right\| \rightarrow 0$ as $N \rightarrow \infty$ and $L=L(N)$. In what follows, we omit the dependence of matrices, projectors, etc. on $N$ and $L$ in our notation.

\subsection{Two general asymptotic results}

We start with two assertions that follow from the inequalities (2.16), (2.22) and (2.17). Denote

$$
\Theta=\Theta_{1} \Theta_{2}=\sqrt{\frac{\nu_{\max }}{\mu_{\max }}} \frac{\mu_{\max }}{\mu_{\min }} .
$$

Proposition 3.1. If $\Theta \rightarrow 0$ as $N \rightarrow \infty$, then

$$
\underset{N}{\limsup } \Theta^{-1}\left\|\mathbf{P}_{0}^{\perp}(\delta)-\mathbf{P}_{0}^{\perp}\right\| \leq 8 C|\delta|
$$

and

$$
\underset{N}{\limsup } \Theta^{-2}\left\|\mathbf{P}_{0}^{\perp}(\delta)-\mathbf{P}_{0}^{\perp}-\delta \mathbf{V}_{0}^{(1)}\right\| \leq C^{\prime} \delta^{2},
$$

where $C=e^{1 / 6} / \sqrt{\pi}$ and $C^{\prime}=2(32 C+1)$.

Thus we see that $\left\|\mathbf{P}_{0}^{\perp}(\delta)-\mathbf{P}_{0}^{\perp}\right\| \rightarrow 0$ for any fixed $\delta$ if $\Theta$ tends to zero. This means that we can treat $\Theta$ in the same sense as the perturbation parameter $\delta$ for fixed $N$. Note that $\Theta \rightarrow 0$ only if $\Theta_{1} \rightarrow 0$. 
At the same time the condition $\Theta_{1} \rightarrow 0$ is not necessary for the convergence $\left\|\mathbf{P}_{0}^{\perp}(\delta)-\mathbf{P}_{0}^{\perp}\right\| \rightarrow 0$. In the next proposition we show that this convergence occurs also in the case $\Theta_{1} \asymp 1$ if the matrices $\mathbf{H}$ and $\mathbf{E}$ are asymptotically biorthogonal and $\Theta_{2}$ is bounded from above. (Note that for positive sequences $a_{n}$ and $b_{n}$ we write $a_{n} \asymp b_{n}$ iff $c_{1} \leq a_{n} / b_{n} \leq c_{2}$ for some constants $c_{1}, c_{2}>0$.) Denote $\Delta=1 / \lim \sup \left(\Theta \Theta_{1}\right)$.

Proposition 3.2. Suppose that $\Delta>0,\left\|\mathbf{H E}^{\mathrm{T}}\right\| / \mu_{\min } \rightarrow 0$, and $\left\|\mathbf{S}_{0} \mathbf{A}^{(2)}\right\| \rightarrow 0$ as $N \rightarrow \infty$. Then for any $\delta$ with $|\delta|<$ $\delta_{0} \stackrel{\text { def }}{=} \Delta / 4$

$$
\begin{aligned}
& \left\|\mathbf{P}_{0}^{\perp}(\delta)-\mathbf{P}_{0}^{\perp}\right\| \\
& \quad=|\delta| O\left(\left\|\mathbf{H} \mathbf{E}^{\mathrm{T}}\right\| / \mu_{\min }+|\delta|\left\|\mathbf{S}_{0} \mathbf{A}^{(2)}\right\|\right)
\end{aligned}
$$

and

$$
\begin{aligned}
& \left\|\mathbf{P}_{0}^{\perp}(\delta)-\mathbf{P}_{0}^{\perp}-\mathbf{L}(\delta)\right\| \\
& \quad=\delta^{2} O\left(\left(\left\|\mathbf{H E}^{\mathrm{T}}\right\| / \mu_{\min }+|\delta|\left\|\mathbf{S}_{0} \mathbf{A}^{(2)}\right\|\right)^{2}\right)
\end{aligned}
$$

provided that $N \geq N_{0}(\delta)$.

Remark 3.1. 1. The operator $\mathbf{L}(\delta)$ is defined in (2.23).

2. If $\Theta \Theta_{1} \asymp 1$, then $\Theta \nrightarrow 0$ and $\delta_{0}<\infty$. Hence the result of Proposition 3.2 is valid only if $|\delta|$ is bounded from above. These restrictions are absent in the case $\Theta \rightarrow 0$ considered in Proposition 3.1.

3. Let $\Theta \Theta_{1} \asymp 1$ and $\Theta_{1} \asymp 1$. Consequently, $\Theta_{2}$ is bounded from above. Applying (2.21) to (3.3) we obtain that $\left\|\mathbf{P}_{0}^{\perp}(\delta)-\mathbf{P}_{0}^{\perp}\right\| \rightarrow 0$ if both $\cos \left(\theta_{r}\right)$ and $\cos \left(\theta_{l}\right)$ tend to zero. Moreover, (3.3) can be rewritten as

$$
\left\|\mathbf{P}_{0}^{\perp}(\delta)-\mathbf{P}_{0}^{\perp}\right\|=|\delta| O\left(\cos \left(\theta_{r}\right)+|\delta| \cos \left(\theta_{l}\right)\right)
$$

and the inequality (3.4) admits similar reformulation.

\subsection{Examples. Rough upper bounds}

To illustrate propositions of the previous subsection we consider several types of "signal" and "noise" series. For a series $\mathrm{F}=\left(f_{0}, \ldots, f_{n}, \ldots\right)$ we define the associated $L \times K$ trajectory matrix in the form

$$
\mathbf{F}=\mathbf{F}_{K, L}=\left(\begin{array}{ccccc}
f_{0} & f_{1} & \ldots & f_{K-2} & f_{K-1} \\
\vdots & \vdots & \ddots & \vdots & \vdots \\
f_{L-1} & f_{L} & \ldots & f_{N-2} & f_{N-1}
\end{array}\right)
$$

and denote the maximal and minimal positive eigenvalues of the matrix $\mathbf{F F}^{\mathrm{T}}$ as $\lambda_{\max }$ and $\lambda_{\min }$, respectively.

In examples we consider the following four types of series.

1. A linear combination of increasing exponents. In this case

$$
f_{n}=\beta_{1} a_{1}^{n}+\cdots+\beta_{p} a_{p}^{n},
$$

where $\beta_{j} \neq 0$ for $1 \leq j \leq p$ and $a_{1}>\cdots>a_{p}>1$. The series (3.5) has rank $p$. For short, we name this series the series of exponential type.
2. A polynomial series. This series is defined by

$$
f_{n}=\gamma_{p} n^{p}+\gamma_{p-1} n^{p-1}+\cdots+\gamma_{1} n+\alpha_{0},
$$

where $\gamma_{p} \neq 0$. The rank of the series (3.6) is $p+1$.

3. An oscillating series with frequencies $\omega_{l}$, positive amplitudes $\gamma_{l}$ and phases $\phi_{l}$. The series has the form

$$
f_{n}=\sum_{l=1}^{p} \gamma_{l} \cos \left(2 \pi \omega_{l} n+\phi_{l}\right)
$$

where $\omega_{l} \in[0,1 / 2]$ and $\omega_{l}<\omega_{j}$ for $l<j$. The rank of the series is $2 p$ if $\omega_{j} \in(0,1 / 2)$ for all $j$. If $\omega_{1}=0$ and $\omega_{p}<1 / 2$ (or if $\omega_{1}>0$ and $\omega_{p}=1 / 2$ ), then the rank is $2 p-1$. If $\omega_{1}=0$ and $\omega_{p}=1 / 2$, then the rank is $2 p-2$.

4. A linear stationary random series. By definition,

$$
f_{n}=\sum_{j=-\infty}^{\infty} c_{j} \varepsilon_{j+n},
$$

where $\varepsilon_{n}$ is the sequence of i.i.d. random variables with $\mathbb{E} \varepsilon_{n}=0, \mathbb{D} \varepsilon_{n}=1$, and $\mathbb{E}\left|\varepsilon_{n}\right|^{3}<\infty$. We assume that $S \stackrel{\text { def }}{=} \sum_{j}\left|c_{j}\right|<\infty$ and $\sum_{j} c_{j}^{2}=1$. Then $\mathbb{E} f_{n}=0$ and $\mathbb{D} f_{n}=1$. Note that the series $\left(f_{0}, \ldots, f_{N}, \ldots\right)$ is not a series of finite rank.

Let us discuss asymptotic properties of the eigenvalues $\lambda_{\max }$ and $\lambda_{\min }$ corresponding to the series (3.5)-(3.8) as $N \rightarrow \infty$ and $L=L(N)$.

Lemma 3.1. 1. For the series of exponential type there exist positive $T_{\max }^{(a)}, T_{\min }^{(a)}$ such that $\lambda_{\max } / a_{1}^{2 N} \rightarrow T_{\max }^{(a)}$ and $\lambda_{\min } / a_{p}^{2 N} \rightarrow T_{\min }^{(a)}$ as $\min (L, K) \rightarrow \infty$. The analogous result holds if either $L=L_{0}=$ const $>p$ or $K=K_{0}=$ const $>p$.

2. For the polynomial series,

a) if $L / N \rightarrow \alpha \in(0,1)$ then $\lambda_{\max } / N^{2 p+2} \rightarrow \Theta_{\max }$ and $\lambda_{\min } / N^{2 p+2} \rightarrow \Theta_{\min }$ for some positive $\Theta_{\max }, \Theta_{\min }$;

b) if either $L$ or $K$ is a constant greater than $p+1$, then $\lambda_{\max } / N^{2 p+1} \rightarrow \Theta_{\max }>0$ and $\lambda_{\min } / N \rightarrow \Theta_{\min }>0$.

3. For the oscillating series, $\lambda_{\max } / L K \rightarrow \Lambda_{\max }>0$ and $\lambda_{\min } / L K \rightarrow \Lambda_{\min }>0$ as $\min (L, K) \rightarrow \infty$. The analogous result holds if either $L=L_{0}=$ const $>d$ or $K=K_{0}=$ const $>d$.

We omit elementary but laborsome proofs of these assertions.

Remark 3.2. 1. Positive constants $T_{\max }^{(a)}, T_{\min }^{(a)}, \Theta_{\max }, \Theta_{\min }$ and $\Lambda_{\max }, \Lambda_{\min }$ can be found in the explicit form and depend both on the parameters of the series and on the behavior of $L=L(N)$.

2. For the oscillating series (3.7) we have $\lambda_{\max } \asymp \lambda_{\min } \asymp$ $N^{2}$ if $L / N \rightarrow \alpha \in(0,1)$ and $\lambda_{\max } \asymp \lambda_{\min } \asymp N$ if either $L$ or $K$ does not depend on $N$.

3. Parameters $a_{j}$ of the series (3.5) are taken positive only for convenience. All results concerning the series of exponential type remain valid under the assumption $\left|a_{1}\right|>$ $\cdots>\left|a_{p}\right|$. 
Table 1. Properties of proximity $\mathbf{P}_{0}^{\perp}(\delta) \approx \mathbf{P}_{0}^{\perp}$ for signals of exponential type and various noise series

\begin{tabular}{||c||c|c|c||}
\hline Type of a noise & Conditions on series & Conditions on $L$ & Rate of convergence \\
\hline Exponential & $b \tau<1$ & - & $(b \tau)^{N}$ \\
\hline Polynomial $(m)$ & $\tau<1$ & $L / N \rightarrow \alpha \in(0,1)$ & $N^{m+1} \tau^{N}$ \\
\hline Polynomial $(m)$ & $\tau<1$ & $\min (L, K)=$ const & $N^{m+1 / 2} \tau^{N}$ \\
\hline Oscillating & $\tau<1$ & - & $\sqrt{L K} \tau^{N}$ \\
\hline
\end{tabular}

Lemma 3.2. Consider the linear stationary series (3.8).

1. If $L \rightarrow \infty$, then there exists an absolute constant $\gamma_{0}$ such that almost surely

$$
\underset{N}{\limsup } \frac{\lambda_{\max }(\omega)}{N \ln N} \leq \gamma_{0} S
$$

2. Assume that $L=L_{0}=$ const and denote $R_{f}(\cdot)$ the covariance function of the series (3.8). Then $\lambda_{\max } / N$ tends almost surely to the maximal eigenvalue $\sigma_{\max }$ of the matrix $\boldsymbol{\Sigma}=\left\{R_{f}(i-j)\right\}_{0 \leq i, j \leq L_{0}-1}$ and $\lambda_{\min } / N$ tends almost surely to the minimal eigenvalue $\sigma_{\min }$ of $\boldsymbol{\Sigma}$.

Both assertions of Lemma 3.2 follow from [21, Proposition 7.1 and Remark 7.1 of Appendix 2].

Remark 3.3. 1. The inequality (3.9) means that for almost all $\omega$ and for any $\gamma^{\prime}>\gamma_{0}$ we get

$$
\lambda_{\max }(\omega)<\gamma^{\prime} S N \ln N
$$

provided that $N>N_{0}\left(\omega, \gamma^{\prime}\right)$.

2. The second statement of Lemma 3.2 holds without the condition $\sup _{n} \mathbb{E}\left|\varepsilon_{n}\right|^{3}<\infty$.

3. If $L=L_{0}$ and $f_{n}=\varepsilon_{n}$ is the "white noise" series, then $\mathbf{F F}^{\mathrm{T}} / K \rightarrow \mathbf{I}_{L_{0}}, \lambda_{\max } / N \rightarrow 1$, and $\lambda_{\min } / N \rightarrow 1$ a.s.

Remark 3.4. Since the series (3.5)-(3.7) are of finite rank, they can serve both as "signal series" and as "noise series". Then we use notation $x_{n}$ or $e_{n}$ instead of $f_{n}$. In the same manner we use notation $\mu_{\max }$ or $\nu_{\max }$ instead of $\lambda_{\max }$ and so on. The stationary series can be chosen only as a "noise series".

\subsubsection{Signals of exponential type}

Consider the signal series defined by (3.5) and some "noise series" E. The following proposition provides a sufficient condition for the convergence $\left\|\mathbf{P}_{0}^{\perp}(\delta)-\mathbf{P}_{0}^{\perp}\right\| \rightarrow 0$ as $N \rightarrow \infty$ in terms of $\nu_{\max }, a_{1}$, and $a_{p}$. Denote $\tau=a_{1} / a_{p}^{2}$.

Proposition 3.3. Let $\Xi_{a} \stackrel{\text { def }}{=} \sqrt{T_{\max }^{(a)}} / T_{\min }^{(a)}$. If $\nu_{\max } \tau^{2 N}=$ $o(1)$ and $\delta \in \mathbb{R}$, then

$$
\begin{gathered}
\underset{N}{\limsup } \nu_{\max }^{-1 / 2} \tau^{-N}\left\|\mathbf{P}_{0}^{\perp}(\delta)-\mathbf{P}_{0}^{\perp}\right\| \leq 8 C \Xi_{a}|\delta| \text { and } \\
\underset{N}{\limsup } \nu_{\max }^{-1} \tau^{-2 N}\left\|\mathbf{P}_{0}^{\perp}(\delta)-\mathbf{P}_{0}^{\perp}-\delta \mathbf{V}_{0}^{(1)}\right\| \leq C^{\prime} \Xi_{a}^{2} \delta^{2} .
\end{gathered}
$$

Remark 3.5. If $p=1$, then $\mu_{\max }=\mu_{\min } \asymp a_{1}^{2 N}, \tau=$ $a_{1}^{-N}<1$, and the condition of Proposition 3.3 can be rewritten as $\nu_{\max }=o\left(a_{1}^{2 N}\right)$.

Table 1 presents several examples related to Proposition 3.3. Let us comment on this table. Three deterministic series are considered as "noise series". (The case of random stationary "noise series" is illustrated in Table 2.)

One noise series is a series of exponential type, $e_{n}=$ $\sum_{l=1}^{m} \gamma_{l} b_{l}^{n}$ with $\gamma_{l} \neq 0$. We assume that $b \stackrel{\text { def }}{=} \max _{1 \leq l \leq m}\left|b_{l}\right|>$ 1 . Two other noise series are a polynomial series of degree $m$ and an oscillating series.

The second column of Table 1 presents the (sufficient) conditions on the signal and noise series for the convergence $\left\|\mathbf{P}_{0}^{\perp}(\delta)-\mathbf{P}_{0}^{\perp}\right\| \rightarrow 0$. These conditions can also depend on the behavior of $L=L(N)$, which is given in the third column of Table 1 . The entry with a dash means that $L$ satisfies only the standard condition $\min (L, K)>d$, where $d$ is the rank of the signal series.

The last column of the table shows the guaranteed rate of convergence $\left\|\mathbf{P}_{0}^{\perp}(\delta)-\mathbf{P}_{0}^{\perp}\right\| \rightarrow 0$. Note that all these results are valid for all $\delta \in \mathbb{R}$ provided that $N$ is big enough.

Table 1 illustrates the first inequality of Proposition 3.3. For the second inequality, the (guaranteed) rate of convergence $\left\|\mathbf{P}_{0}^{\perp}(\delta)-\mathbf{P}_{0}^{\perp}-\delta \mathbf{V}_{0}^{(1)}\right\| \rightarrow 0$ is obtained as the square of the expressions given in the last column of the table.

Remark 3.6. It is easy to see that Proposition 3.3 (and therefore, Proposition 3.1) can give only an upper bound for the true rate of convergence $\mathbf{P}_{0}^{\perp}(\delta) \rightarrow \mathbf{P}_{0}^{\perp}$. For example, for $x_{n}=a^{n}$ with $a>1$ and the constant "noise series" $e_{n} \equiv 1$, we obtain the equality $\left\|\mathbf{P}_{0}^{\perp}(\delta)-\mathbf{P}_{0}^{\perp}\right\|=O\left(N a^{-N}\right)$ in the case $L \sim K$, see Table 1 .

On the other hand, Proposition 4.1 of Section 4.1 affirms that $\left\|\mathbf{P}_{0}^{\perp}(\delta)-\mathbf{P}_{0}^{\perp}\right\| \asymp \sqrt{N} a^{-N}$. The reason of this drawback is that Proposition 3.1 can ignore the existing asymptotic orthogonalities of signal and noise matrices.

\subsubsection{Oscillating signal and noise series}

Consider the signal of oscillating type with different frequencies $\omega_{l 1} \in[0,1 / 2]$, positive amplitudes $\alpha_{l}$, and phases $\phi_{l 1}, l=1, \ldots, p$. Assume that the "noise series" is also oscillating. Namely, let

$$
e_{n}=\sum_{j=1}^{m} \beta_{j} \cos \left(2 \pi \omega_{j 2} n+\phi_{j 2}\right),
$$


Table 2. Properties of proximity $\mathbf{P}_{0}^{\perp}(\delta) \approx \mathbf{P}_{0}^{\perp}$ for various signals and random noise series

\begin{tabular}{||c||c|c|c|c|c||}
\hline Type of a signal & Type of a noise & Conditions on $L$ & Conditions on $\delta$ & Rate of conv. & Main term \\
\hline Exponential & Linear stationary & $L \rightarrow \infty$ & - & $\sqrt{N \ln N} \tau^{N}$ & $\delta \mathbf{V}_{0}^{(1)}$ \\
\hline Exponential & Linear stationary & $L=$ const & - & $\sqrt{N} \tau^{N}$ & $\delta \mathbf{V}_{0}^{(1)}$ \\
\hline Polynomial $(p)$ & Linear stationary & $L / N \rightarrow \alpha \in(0,1)$ & - & $\sqrt{\ln N} N^{-p-1 / 2}$ & $\delta \mathbf{V}_{0}^{(1)}$ \\
\hline Oscillating & Linear stationary & $L / N \rightarrow \alpha \in(0,1)$ & - & $\sqrt{\ln N / N}$ & $\delta \mathbf{V}_{0}^{(1)}$ \\
\hline Oscillating & White noise & $L=$ const & $|\delta|<\delta_{0}$ & $\sqrt{\ln \ln N / N}$ & $\mathbf{T}(\delta)$ \\
\hline
\end{tabular}

where $\beta_{j}>0$ and $\omega_{j 1} \in[0,1 / 2]$. In addition, let $\omega_{l 1} \neq \omega_{j 2}$ for all $l, j$.

If $\min (L, K) \rightarrow \infty$, then $\mu_{\max } \sim \Lambda_{\max }^{(s)} L K$, $\mu_{\min } \sim \Lambda_{\min }^{(s)} L K, \quad \nu_{\max } \sim \Lambda_{\max }^{(n)} L K$, and $\Theta \Theta_{1}=$ $\nu_{\max } / \mu_{\min } \rightarrow 1 / \Delta \stackrel{\text { def }}{=} \Lambda_{\max }^{(n)} / \Lambda_{\min }^{(s)}$. Consequently, the result of Proposition 3.2 is valid for any $\delta$ such that $|\delta|<\delta_{0}=\Delta / 4$ provided that $N$ is big enough.

Calculations show that $\left\|\mathbf{H E}^{\mathrm{T}}\right\| / \mu_{\min }=O(1 / K)$ and $\left\|\mathbf{S}_{0} \mathbf{A}^{(2)}\right\|=O(1 / L)$. Therefore, it follows from (3.3) and (3.4) that

$$
\begin{aligned}
& \left\|\mathbf{P}_{0}^{\perp}(\delta)-\mathbf{P}_{0}^{\perp}\right\|=|\delta| O(1 / \min (L, K)) \text { and } \\
& \left\|\mathbf{P}_{0}^{\perp}(\delta)-\mathbf{P}_{0}^{\perp}-\mathbf{L}(\delta)\right\|=\delta^{2} O\left(1 / \min \left(L^{2}, K^{2}\right)\right) .
\end{aligned}
$$

\subsubsection{Random stationary "noise series"}

Let $\varepsilon_{n}(n \geq 0)$ be a sequence of i.i.d. random variables defined on a probability space $(\Omega, \mathcal{F}, \mathbb{P})$. Assume that $\mathbb{E} \varepsilon_{n}=0$, $\mathbb{D} \varepsilon_{n}=1$, and $\mathbb{E}\left|\varepsilon_{n}\right|^{3}<\infty$.

In the present section we consider several signal series $x_{n}$ and the linear stationary series $e_{n}$ as the "noise series". (The series $e_{n}$ is determined by the right-hand side of (3.8).) In this case $\mathbf{P}_{0}^{\perp}(\delta)$ is a random operator dependent on $\omega \in \Omega$. Moreover, for fixed $(\delta, N, L)$ the condition (2.1) of Theorem 2.1 holds only with a certain probability.

As in Section 3.2.1, we further present general statements and then consider several examples.

Proposition 3.4. 1. Suppose that $L \rightarrow \infty$. If $\mu_{\max } / \mu_{\min }^{2}=$ $o(1 /(N \ln N))$, then there exist $\Omega^{\prime} \in \mathcal{F}$ with $\mathbb{P}\left(\Omega^{\prime}\right)=1$ and an absolute constant $\gamma_{0}$ such that for any $\omega \in \Omega^{\prime}$ and any $\delta$

$$
\underset{N}{\limsup } \frac{\mu_{\min }}{\sqrt{\mu_{\max } N \ln N}}\left\|\mathbf{P}_{0}^{\perp}(\delta)-\mathbf{P}_{0}^{\perp}\right\| \leq 8 C \sqrt{\gamma_{0} S}|\delta| \text { and }
$$$$
\underset{N}{\limsup } \frac{\mu_{\min }^{2}}{\mu_{\max } N \ln N}\left\|\mathbf{P}_{0}^{\perp}(\delta)-\mathbf{P}_{0}^{\perp}-\delta \mathbf{V}_{0}^{(1)}\right\| \leq C^{\prime} \gamma_{0} S \delta^{2},
$$

where $C, C^{\prime}$ are defined in Proposition 3.1 and $S=\sum_{j}\left|c_{j}\right|$.

2. If $L=L_{0}=$ const and $\mu_{\max } / \mu_{\min }^{2}=o(1 / N)$, then there exists a certain $\Omega^{\prime} \in \mathcal{F}$ with $\mathbb{P}\left(\Omega^{\prime}\right)=1$ such that for any $\omega \in \Omega^{\prime}$ and for any $\delta$

$$
\begin{gathered}
\underset{N}{\limsup } \frac{\mu_{\min }}{\sqrt{N \mu_{\max }}}\left\|\mathbf{P}_{0}^{\perp}(\delta)-\mathbf{P}_{0}^{\perp}\right\| \leq 8 C \sqrt{\sigma_{\max }}|\delta| \text { and } \\
\limsup _{N} \frac{\mu_{\min }^{2}}{N \mu_{\max }}\left\|\mathbf{P}_{0}^{\perp}(\delta)-\mathbf{P}_{0}^{\perp}-\delta \mathbf{V}_{0}^{(1)}\right\| \leq C^{\prime} \sigma_{\max } \delta^{2},
\end{gathered}
$$

where $\sigma_{\max }$ is the maximal eigenvalue of the matrix $\boldsymbol{\Sigma}=$ $\left\{R_{e}(i-j)\right\}_{0 \leq i, j<L_{0}}$ and $R_{e}(\cdot)$ stands for the covariation function of $e_{n}$.

The proof of Proposition 3.4 can be found in [21, Appendix 1].

The case of oscillating signal series and white-noise "noise series" $e_{n}=\varepsilon_{n}$ is of particular importance.

Proposition 3.5. Consider the oscillating signal series (3.7) and i.i.d. "noise series" $e_{n}=\varepsilon_{n}$ defined on a probability space $(\Omega, \mathcal{F}, \mathbb{P})$. Assume that $\mathbb{E} \varepsilon_{n}=0$, $\mathbb{D} \varepsilon_{n}=1$, and $\mathbb{E}\left|\varepsilon_{n}\right|^{3}<\infty$.

If $L=L_{0}=$ const, $\delta_{0}^{2}<L_{0} \Lambda_{\min } / 4$, and $N \rightarrow \infty$, then there exists $\Omega^{\prime} \in \mathcal{F}$ with $\mathbb{P}\left(\Omega^{\prime}\right)=1$ such that for any $\delta \in$ $\left(-\delta_{0}, \delta_{0}\right)$ and any $\omega \in \Omega^{\prime}$

$$
\limsup _{N} \sqrt{N(\ln \ln N)^{-1}}\left\|\mathbf{P}_{0}^{\perp}(\delta)-\mathbf{P}_{0}^{\perp}\right\|<c^{\prime}|\delta|
$$

and

$$
\underset{N}{\limsup } N(\ln \ln N)^{-1}\left\|\mathbf{P}_{0}^{\perp}(\delta)-\mathbf{P}_{0}^{\perp}-\mathbf{T}(\delta)\right\|<c^{\prime \prime} \delta^{2}
$$

where positive constants $c^{\prime}$ and $c^{\prime \prime}$ depend on $L_{0}, \delta_{0}$, and parameters of the series (3.7).

The proof of Proposition 3.5 can be found in [21, Appendix 1].

Table 2 illustrates Propositions 3.4 and 3.5. Examples include signals of exponential type with $\tau=a_{1} / a_{p}^{2}<1$, polynomial signals of order $p$ and oscillating signals. Linear stationary series and white noise series are taken as "noise series".

The fifth column of Table 2 describes the guaranteed rate of a.s. convergence $\left\|\mathbf{P}_{0}^{\perp}(\delta)-\mathbf{P}_{0}^{\perp}\right\| \rightarrow 0$. The last column presents the main terms of the approximation $\mathbf{P}_{0}^{\perp}(\delta) \approx \mathbf{P}_{0}^{\perp}$. More detailed information on examples given in Tables 1 and 2 can be found in [21, Sect. 3.2].

\section{EXAMPLES. PRECISE ASYMPTOTIC RESULTS}

The results of Section 3 can provide overestimated upper bound for the rate of convergence $\left\|\mathbf{P}_{0}^{\perp}(\delta)-\mathbf{P}_{0}^{\perp}\right\| \rightarrow 0$, see Remark 3.6. Moreover, the operators $\mathbf{W}_{1}(\delta), \mathbf{L}(\delta)$, and $\mathbf{T}(\delta)$ 
determined by (2.9), (2.23), and (2.27) respectively can serve only as candidates for the main term of this convergence.

In this section we present several examples of "signal" and "noise" series for which both true rates of convergence $\left\|\mathbf{P}_{0}^{\perp}(\delta)-\mathbf{P}_{0}^{\perp}\right\| \rightarrow 0$ and the related main terms are obtained explicitly.

These examples are rather simple and have minor practical value. In particular, we consider signal series of rank 1 . Then all relevant operators are derived in accessible form.

In addition, the choice of examples corresponds to different conditions for the convergence $\left\|\mathbf{P}_{0}^{\perp}(\delta)-\mathbf{P}_{0}^{\perp}\right\| \rightarrow 0$. More precisely, the example of Section 4.1 describes the case of "big signal" and "small noise" while the Section 4.2.1 is devoted to the asymptotic orthonormalities. The case of white noise "noise series" is considered in Section 4.2.2.

\subsection{Exponential signal series and constant "noise series"}

Consider the "signal series" $x_{n}=a^{n}$ with $a>1$ and the "noise series" $e_{n} \equiv 1$. Let $W_{j}=\left(1, a, \ldots, a^{j-1}\right)^{\mathrm{T}}, E_{j}=$ $(1, \ldots, 1)^{\mathrm{T}} \in \mathbb{R}^{j}$, and $\beta_{j}=W_{j}^{\mathrm{T}} E_{j}$. Then $\left\|E_{j}\right\|=\sqrt{j}$ and $\left\|W_{j}\right\|=\sqrt{\left(a^{2 j}-1\right) /\left(a^{2}-1\right)}$.

Since matrices $\mathbf{H}=W_{L} W_{K}^{\mathrm{T}}$ and $\mathbf{E}=E_{L} E_{K}^{\mathrm{T}}$ have rank 1, then $\nu_{\max }=\left\|E_{L}\right\|^{2}\left\|E_{K}\right\|^{2}=L K$ and $\mu_{\max }=\mu_{\min }=$ $\left\|W_{L}\right\|^{2}\left\|W_{K}\right\|^{2} \asymp a^{2 N}$ as $N \rightarrow \infty$. Thus, applying Proposition 3.1 with $\Theta \sim \sqrt{L K} / a^{N} \rightarrow 0$ and using (3.1) and (3.2), we obtain that for any $\delta$ and $N>N_{0}(\delta)$

$$
\left\|\mathbf{P}_{0}^{\perp}(\delta)-\mathbf{P}_{0}^{\perp}\right\|=|\delta| O\left(\sqrt{L K} a^{-N}\right)
$$

and

$$
\left\|\mathbf{P}_{0}^{\perp}(\delta)-\mathbf{P}_{0}^{\perp}-\delta \mathbf{V}_{0}^{(1)}\right\|=\delta^{2} O\left(L K a^{-2 N}\right),
$$

where $\mathbf{V}_{0}^{(1)}$ is defined in (2.7).

The following proposition presents precise variants of (4.1) and (4.2). Denote

$$
H(a, L)=\frac{(a+1)}{a} \frac{a^{L} \sqrt{L}\left\|W_{L}\right\|^{2}-\beta_{L}^{2}}{\left\|W_{L}\right\|^{2}} .
$$

Proposition 4.1. Let $\mathbf{Z}_{0}^{(1)}$ stand for $\mathbf{E} \mathbf{H}^{\mathrm{T}} \mathbf{S}_{0}+\mathbf{S}_{0} \mathbf{H E}^{\mathrm{T}}$. 1. If $L / N \rightarrow \alpha \in(0,1)$, then

$$
\begin{gathered}
\frac{a^{N}}{\sqrt{N}}\left\|\mathbf{P}_{0}^{\perp}(\delta)-\mathbf{P}_{0}^{\perp}\right\| \rightarrow|\delta| \frac{\alpha(a+1) \sqrt{a^{2}-1}}{a} \text { and } \\
a^{N}\left\|\mathbf{P}_{0}^{\perp}(\delta)-\mathbf{P}_{0}^{\perp}-\delta \mathbf{Z}_{0}^{(1)}\right\| \rightarrow|\delta| \frac{2(a+1)^{2}}{a} .
\end{gathered}
$$

2. If $K=K_{0}=$ const and $N \rightarrow \infty$, then

$$
\begin{aligned}
& \frac{a^{N}}{\sqrt{N}}\left\|\mathbf{P}_{0}^{\perp}(\delta)-\mathbf{P}_{0}^{\perp}\right\| \rightarrow|\delta| \frac{(a+1) \sqrt{a^{2}-1}}{a\left(1-a^{-K_{0}}\right)} \text { and } \\
& a^{N}\left\|\mathbf{P}_{0}^{\perp}(\delta)-\mathbf{P}_{0}^{\perp}-\delta \mathbf{Z}_{0}^{(1)}\right\| \rightarrow|\delta| \frac{2(a+1)^{2}}{a\left(1-a^{-K_{0}}\right)} .
\end{aligned}
$$

3. If $L=L_{0}=$ const and $N \rightarrow \infty$, then

$$
\begin{aligned}
& a^{N}\left\|\mathbf{P}_{0}^{\perp}(\delta)-\mathbf{P}_{0}^{\perp}\right\| \rightarrow|\delta| H\left(a, L_{0}\right) \quad \text { while } \\
& \left\|\mathbf{P}_{0}^{\perp}(\delta)-\mathbf{P}_{0}^{\perp}-\delta \mathbf{V}_{0}^{(1)}\right\|=\delta^{2} O\left(N a^{-2 N}\right) .
\end{aligned}
$$

Remark 4.1. 1. Matrices $\mathbf{V}_{0}^{(1)}$ and $\mathbf{Z}_{0}^{(1)}$ have the following explicit form, $\mathbf{V}_{0}^{(1)}=\mathbf{Z}_{0}^{(1)}-\mathbf{Z}_{0}^{(2)}$, where

$$
\begin{aligned}
& \mathbf{z}_{0}^{(1)}=\beta_{K} \frac{E_{L} W_{L}^{\mathrm{T}}+W_{L} E_{L}^{\mathrm{T}}}{\left\|W_{L}\right\|^{2}\left\|W_{K}\right\|^{2}} \text { and } \\
& \mathbf{z}_{0}^{(2)}=\beta_{L} \beta_{K} \frac{W_{L} W_{L}^{\mathrm{T}}}{\left\|W_{L}\right\|^{4}\left\|W_{K}\right\|^{2}} .
\end{aligned}
$$

2. Proposition 4.1 shows that the best rate of convergence of $\mathbf{P}_{0}^{\perp}(\delta)$ to $\mathbf{P}_{0}^{\perp}$ is achieved under the choice $L=L_{0}=$ const. This rate is equal to $a^{-N}$ and the main term of approximation $\mathbf{P}_{0}^{\perp}(\delta) \approx \mathbf{P}_{0}^{\perp}$ is the linear term $\delta \mathbf{V}_{0}^{(1)}$. In this case the norms of operators $\mathbf{Z}_{0}^{(1)}$ and $\mathbf{Z}_{0}^{(2)}$ have the same order of growth and therefore we cannot reduce $\mathbf{V}_{0}^{(1)}$ to $\mathbf{Z}_{0}^{(1)}$ as it was done in the case $L \rightarrow \infty$.

\subsection{Constant series as a signal}

In this section we consider the constant series $x_{n}=1$ as a signal. Let $W_{j}=(1, \ldots, 1)^{\mathrm{T}} \in \mathbb{R}^{j}$. Then the $L \times K$ trajectory matrix of the series has the form $\mathbf{H}=W_{L} W_{K}^{\mathrm{T}}$ and $\|\mathbf{H}\|=\sqrt{L K}$. Therefore, $d=1$ and $\mu_{\max }=\mu_{\min }=L K$.

\subsubsection{Saw series as a "noise"}

Consider the saw series $e_{n}=(-1)^{n}$ as a "noise". Then $\mathbf{E}=E_{L} E_{K}^{\mathrm{T}}$, where $E_{j}=\left(e_{0}, \ldots, e_{j-1}\right)^{\mathrm{T}}$. As earlier, let $\beta_{j}=$ $W_{j}^{\mathrm{T}} E_{j}$. Note that $\beta_{j}=0$ for even $j$ and $\beta_{j}=1$ for odd $j$.

Since $\nu_{\max }=L K$, then $\Theta_{1}=\Theta_{2}=1$ and we cannot apply Proposition 3.1. Nevertheless we can use Theorem 2.5 and Proposition 3.2 to obtain conditions for the convergence $\left\|\mathbf{P}_{0}^{\perp}(\delta)-\mathbf{P}_{0}^{\perp}\right\| \rightarrow 0$ for small $\delta$ and $N \rightarrow \infty$. Since $d=1$ we impose $\min (L, K)>1$.

Proposition 4.2. Let $|\delta|<1 / 2$. If $L$ and $K$ are both even, then $\mathbf{P}_{0}^{\perp}(\delta)=\mathbf{P}_{0}^{\perp}$, otherwise

$$
\begin{aligned}
& \left\|\mathbf{P}_{0}^{\perp}(\delta)-\mathbf{P}_{0}^{\perp}\right\| \\
& =|\delta| \begin{cases}O\left(K^{-1}\right) & \text { for odd } K, \text { even } L \\
& \text { and } K \rightarrow \infty, \\
O\left(L^{-1}\right) & \text { for odd } L, \text { even } K \\
O\left(L^{-1}+K^{-1}\right) & \text { and } L \rightarrow \infty, \\
& \text { for } L, K \text { both odd } \min (L, K) \rightarrow \infty .\end{cases}
\end{aligned}
$$

Remark 4.2. Since the series $E$ is periodic with period 2, the different results for odd and even $L, K$ are not amazing. In view of [13, Ch. $1 \S 1.6 .2]$, the choice of window length as an integer multiple of the period of periodical component of the series can essentially improve separability. Here we have got the formal affirmation of this principle. 
The case for even $L, K$ exactly corresponds to biorthogonality of matrices $\mathbf{H}$ and $\mathbf{E}$, see the discussion in Section 2.2.1. Therefore, no additional restrictions on $L, K$ are needed.

Now our goal is to study the main term of the difference $\mathbf{P}_{0}^{\perp}(\delta)-\mathbf{P}_{0}^{\perp}$ by applying Theorem 2.5 and Proposition 2.2.

Consider fixed $\delta$ such that $|\delta|<1 / 2$. Denote

$$
E_{L, w}=E_{L}-\beta_{L} W_{L} / L
$$

$W_{E}=W_{L} E_{L, w}^{\mathrm{T}}+E_{L, w} W_{L}^{\mathrm{T}}$, and

$$
\mathbf{M}(\delta)= \begin{cases}\frac{\delta}{1-\delta^{2}} \frac{W_{E}}{L K} & \text { for odd } K \\ \frac{\delta^{2}}{1-\delta^{2}} \frac{W_{E}}{L \sqrt{L^{2}-1}} & \text { for odd } L \\ \frac{\delta}{1-\delta^{2}}\left(\frac{1}{K}+\frac{\delta}{L}\right) \frac{W_{E}}{\sqrt{L^{2}-1}} & \text { and even } K, \quad \text { both odd } K\end{cases}
$$

Proposition 4.3. The norm of the operator $\mathbf{M}(\delta)$ is

$$
\|\mathbf{M}(\delta)\|= \begin{cases}\frac{|\delta|}{1-\delta^{2}} \frac{1}{K} & \text { for odd } K \text { and even } L \\ \frac{\delta^{2}}{1-\delta^{2}} \frac{1}{L} & \text { for odd } L \text { and even } K \\ \frac{|\delta|}{1-\delta^{2}}\left|\frac{1}{K}+\frac{\delta}{L}\right| & \text { for } K \text { and } L \text { both odd }\end{cases}
$$

while

$$
\begin{aligned}
& \left\|\mathbf{P}_{0}^{\perp}(\delta)-\mathbf{P}_{0}^{\perp}-\mathbf{M}(\delta)\right\| \\
& = \begin{cases}O\left(K^{-2}\right) & \text { for odd } K, \text { even } L \\
O\left(L^{-2}\right) & \text { and } K \rightarrow \infty, \\
& \text { for odd } L, \text { even } K \\
O\left(L^{-2}+K^{-2}\right) & \text { and } L \rightarrow \infty, \\
& \text { for } L, K \text { both odd } \min (L, K) \rightarrow \infty .\end{cases}
\end{aligned}
$$

Remark 4.3. 1. Unlike to Proposition 4.1, Proposition 4.3 shows that the main term $\mathbf{M}(\delta)$ is nonlinear in $\delta$.

2. The norm (4.5) indicates the distinctions between positive and negative $\delta$ in the case of odd $L, K$. For example, if $\delta<0$ and $L=-\delta K$, then $\left\|\mathbf{P}_{0}^{\perp}(\delta)-\mathbf{P}_{0}^{\perp}\right\| \asymp L^{-2}$ whereas this norm has the order $L^{-1}$ for any positive $\delta$ and $L \sim K$.

\subsubsection{White noise as the "noise series"}

In this section we study the difference $\mathbf{P}_{0}^{\perp}(\delta)-\mathbf{P}_{0}^{\perp}$ for the constant signal $x_{n}=1$ and the white-noise "noise series" $e_{n}=\varepsilon_{n}$ where $e_{n}$ are i.i.d. random variables $\varepsilon_{n}$ such that $\mathbb{E} \varepsilon_{n}=0, \mathbb{E} \varepsilon_{n}^{2}=1$ and $\mathbb{E} \varepsilon_{n}^{4}<\infty$.

The a.s. behavior of $\left\|\mathbf{P}_{0}^{\perp}(\delta)-\mathbf{P}_{0}^{\perp}\right\|$ as $N \rightarrow \infty$ is already studied in Section 3.2. Now we derive the main term of the difference $\mathbf{P}_{0}^{\perp}(\delta)-\mathbf{P}_{0}^{\perp}$ as $N \rightarrow \infty$ and $L=L_{0}=$ const in terms of a suitable version of the central limit theorem.

Denote $\Psi_{L_{0}}=\left\{\psi_{i j}\right\}_{i, j=0}^{L_{0}-1}$ a random symmetric $L_{0} \times L_{0}$ Toeplitz matrix where $\psi_{0 j}\left(j=0, \ldots, L_{0}-1\right)$ are independent, $\psi_{00} \in \mathrm{N}\left(0, \mathbb{E} \varepsilon^{4}-1\right)$, and $\psi_{0 j} \in \mathrm{N}(0,1)$ for $j \geq 1$. (Note that $\xi \in \mathrm{N}\left(a, \sigma^{2}\right)$ means that $\xi$ has a normal distribution, $\mathbb{E} \xi=a$, and $\mathbb{D} \xi=\sigma^{2}$.)

Proposition 4.4. For fixed $L_{0}, \delta$ such that $\delta^{2}<L_{0} / 4$ denote

$$
\Omega_{N}=\left\{\omega \in \Omega \text { such that }\|\mathbf{B}(\delta)\|<\mu_{\min } / 2\right\} .
$$

Then $\mathbb{P}\left(\Omega_{N}\right) \rightarrow 1$ as $N \rightarrow \infty$ and

$$
\begin{aligned}
& \mathcal{L}\left(\sqrt{N}\left(\mathbf{P}_{0}^{\perp}(\delta)-\mathbf{P}_{0}^{\perp}\right) \mid \Omega_{N}\right) \\
& \Longrightarrow \mathcal{L}\left(\frac{\delta^{2}}{L_{0}}\left(\mathbf{P}_{0}^{\perp} \Psi_{L_{0}} \mathbf{P}_{0}+\mathbf{P}_{0} \Psi_{L_{0}} \mathbf{P}_{0}^{\perp}\right)\right),
\end{aligned}
$$

where " $\Longrightarrow "$ stands for weak convergence of distributions and $\mathcal{L}(\xi)$ means the distribution of the random vector $\xi$.

The proof of Proposition 4.4 can be found in [21, Sect. 7.1 of Appendix 1].

Remark 4.4. 1. Roughly speaking, Proposition 4.4 shows that the main term of the difference $\mathbf{P}_{0}^{\perp}(\delta)-\mathbf{P}_{0}^{\perp}$ has the asymptotic form

$$
\frac{\delta^{2}}{L_{0} \sqrt{N}}\left(\mathbf{P}_{0}^{\perp} \Psi_{L_{0}} \mathbf{P}_{0}+\mathbf{P}_{0} \Psi_{L_{0}} \mathbf{P}_{0}^{\perp}\right) .
$$

2. Since $L_{0}$ is fixed and the norm $\|\cdot\|$ is a continuous functional on the space of $L_{0} \times L_{0}$ matrices, then

$$
\begin{aligned}
& \mathcal{L}\left(\sqrt{N}\left\|\mathbf{P}_{0}^{\perp}(\delta)-\mathbf{P}_{0}^{\perp}\right\| \mid \Omega_{N}\right) \\
& \Longrightarrow \mathcal{L}\left(\frac{\delta^{2}}{L_{0}}\left\|\mathbf{P}_{0}^{\perp} \Psi_{L_{0}} \mathbf{P}_{0}+\mathbf{P}_{0} \Psi_{L_{0}} \mathbf{P}_{0}^{\perp}\right\|\right)
\end{aligned}
$$

under the conditions of Proposition 4.4.

\section{ON THE WAY TO APPLICATIONS}

In the present section we briefly describe the application of the previous results to several methods of Signal Subspace Analysis. Section 5.1 is devoted to the approximation of linear recurrent formulas governing the signal and in Section 5.2 we study the real-valued variant of Least-Square ESPRIT. We demonstrate that both methods asymptotically converge under the assumption $\left\|\mathbf{P}_{0}^{\perp}(\delta)-\mathbf{P}_{0}^{\perp}\right\| \rightarrow 0$.

In Section 5.2 we discuss the reconstruction stage of SSA. Though the precision of SSA can not be described only in terms of the proximity of the perturbed and unperturbed projectors, the obtained results help to reformulate the problem in a more transparent form.

\subsection{Approximations of linear recurrent formulas}

Let the signal series $\mathrm{F}=\left(x_{0}, \ldots, x_{n}, \ldots\right)$ be governed by a linear recurrent formula (LRF)

$$
x_{n}=\sum_{k=1}^{d} b_{k} x_{n-k}, \quad n \geq d
$$


and suppose that (5.1) is the minimal LRF for the series F. In particular, this means that $b_{d} \neq 0$.

For $L, K>d$ let $\mathbf{H}$ stand for the trajectory $L \times K$ matrix of the series $\mathrm{F}$. Then $\operatorname{rank} \mathbf{H}=d$. Consider the signal subspace $\mathbb{U}_{0}^{\perp}$ and the corresponding projector $\mathbf{P}_{0}^{\perp}$. Denote $\mathfrak{e}_{L}=(0,0, \ldots, 0,1)^{\mathrm{T}} \in \mathbb{R}^{L}$. As proved in [13, Sect. 5.2], $\mathbf{P}_{0} \mathfrak{e}_{L} \neq 0$. (Note that $\left\|\mathbf{P}_{0} \mathfrak{e}_{L}\right\|$ is a cosine between the vector $\mathfrak{e}_{L}$ and the linear space $\mathbb{U}_{0}$.)

Let $\mathbf{G}_{L}$ stand for the $(L-1) \times L$ matrix

$$
\mathbf{G}_{L}=\left(\begin{array}{cccccc}
1 & 0 & \ldots & 0 & 0 & 0 \\
0 & 1 & \ldots & 0 & 0 & 0 \\
\vdots & \vdots & \ddots & \vdots & \vdots & \vdots \\
0 & 0 & \ldots & 1 & 0 & 0 \\
0 & 0 & \ldots & 0 & 1 & 0
\end{array}\right)
$$

and denote

$$
R=\left(a_{L-1}, \ldots, a_{1}\right)^{\mathrm{T}}=-\frac{1}{\left\|\mathbf{P}_{0} \mathfrak{e}_{L}\right\|^{2}} \mathbf{G}_{L} \mathbf{P}_{0} \mathfrak{e}_{L} .
$$

Due to [13, Th. 5.2],

$$
x_{n}=\sum_{k=1}^{L-1} a_{k} x_{n-k}, \quad n \geq L .
$$

Note that analogous expressions are known from early 80s, see, for example, [22]. It is clear that (5.4) coincides with (5.1) for $L=d+1$.

The formula (5.3) is used to derive an approximation of $R$ in the case when the signal series $\mathrm{F}$ is corrupted by an additive noise series $\mathrm{E}$. In other words, if $\mathrm{F}(\delta)=\mathrm{F}+\delta \mathrm{E}$, then the natural form of this approximation has the form

$$
R(\delta)=-\frac{1}{\left\|\mathbf{P}_{0}(\delta) \mathfrak{e}_{L}\right\|^{2}} \mathbf{G}_{L} \mathbf{P}_{0}(\delta) \mathfrak{e}_{L}
$$

If $\mathbf{P}_{0}(\delta)$ is close to $\mathbf{P}_{0}$, then $R(\delta)$ must be close to $R$.

Proposition 5.1. Let $\Delta \mathbf{P}(\delta)$ stand for $\left\|\mathbf{P}_{0}^{\perp}(\delta)-\mathbf{P}_{0}^{\perp}\right\|$. Denote $\vartheta=\left\|\mathbf{P}_{0}^{\perp} \mathfrak{e}_{L}\right\|$ and suppose that $\Delta \mathbf{P}(\delta)<\left\|\mathbf{P}_{0} \mathfrak{e}_{L}\right\|=$ $\sqrt{1-\vartheta^{2}}$. Then

$$
\|R(\delta)-R\| \leq \frac{\Delta \mathbf{P}(\delta)}{1-\vartheta^{2}}\left(1-\frac{\Delta \mathbf{P}(\delta)}{\sqrt{1-\vartheta^{2}}}\right)^{-2}\left(1+\frac{2}{\sqrt{1-\vartheta^{2}}}\right) .
$$

Proposition 5.1 gives sufficient conditions for the convergence $\|R(\delta)-R\| \rightarrow 0$ in terms of $\left\|\mathbf{P}_{0}^{\perp}(\delta)-\mathbf{P}_{0}^{\perp}\right\|$ and $\left\|\mathbf{P}_{0} \mathfrak{e}_{L}\right\|$. Namely, if $\left\|\mathbf{P}_{0}^{\perp}(\delta)-\mathbf{P}_{0}^{\perp}\right\| \rightarrow 0$ and if $\left\|\mathbf{P}_{0} \mathfrak{e}_{L}\right\|$ is separated from zero, then $\|R(\delta)-R\| \rightarrow 0$.

The second condition automatically holds in the case $L=$ const $>d$ (then both $\mathfrak{e}_{L}$ and $\mathbf{P}_{0}$ do not depend on $N$ ). It is not difficult to show (we omit proofs for brevity) that $\left\|\mathbf{P}_{0} \mathfrak{e}_{L}\right\|$ is separated from zero as $L \rightarrow \infty$ for signals of exponential type, polynomial signals and oscillating signals. Therefore, in these cases (5.5) takes the form $\|R(\delta)-R\|=$ $O\left(\left\|\mathbf{P}_{0}^{\perp}(\delta)-\mathbf{P}_{0}^{\perp}\right\|\right)$. Examples of Section 3.2 refine this general assertion for a number of "signal" and "noise" series.

\subsection{LS-ESPRIT for real-valued signals}

As in Section 5.1, we suppose that the signal series $\mathrm{F}=\left(x_{0}, \ldots, x_{n}, \ldots\right)$ is governed by the linear recurrent formula (5.1) with $b_{d} \neq 0$. It is well-known that the general solution of (5.1) is expressed through the roots of the characteristic polynomial $P_{d}(\lambda)=\lambda_{d}-\sum_{k=1}^{d} b_{k} \lambda^{d-k}$.

Assume that $L, K>d$ and consider the $L \times K$ trajectory matrix $\mathbf{H}$ of the series F. As in previous sections, let $\mathbb{U}_{0}^{\perp}$ stand for the linear space spanned by columns of the matrix H. For a certain basis $U_{1}, \ldots, U_{d}$ of $\mathbb{U}_{0}^{\perp}$ denote $\mathbf{U}=\left[U_{1}\right.$ : $\left.\ldots: U_{d}\right]$. Lastly, let the matrix $\mathbf{G}_{L}$ be defined by (5.2) and denote the $(L-1) \times L$ matrix $\mathbf{G}^{(L)}$ by

$$
\mathbf{G}^{(L)}=\left(\begin{array}{cccccc}
0 & 1 & 0 & \ldots & 0 & 0 \\
0 & 0 & 1 & \ldots & 0 & 0 \\
\vdots & \vdots & \vdots & \ddots & \vdots & \vdots \\
0 & 0 & 0 & \ldots & 1 & 0 \\
0 & 0 & 0 & \ldots & 0 & 1
\end{array}\right) .
$$

Note that

$$
\begin{aligned}
\mathbf{F}_{1} \stackrel{\text { def }}{=} \mathbf{G}_{L}^{\mathrm{T}} \mathbf{G}_{L}=\left(\begin{array}{ccccc}
1 & 0 & \ldots & 0 & 0 \\
0 & 1 & \ldots & 0 & 0 \\
\vdots & \vdots & \ddots & \vdots & \vdots \\
0 & 0 & \ldots & 1 & 0 \\
0 & 0 & \ldots & 0 & 0
\end{array}\right) \text { and } \\
\mathbf{F}_{2} \stackrel{\text { def }}{=} \mathbf{G}_{L}^{\mathrm{T}} \mathbf{G}^{(L)}=\left(\begin{array}{cccccc}
0 & 1 & 0 & \ldots & 0 & 0 \\
0 & 0 & 1 & \ldots & 0 & 0 \\
\vdots & \vdots & \vdots & \ddots & \vdots & \vdots \\
0 & 0 & 0 & \ldots & 0 & 1 \\
0 & 0 & 0 & \ldots & 0 & 0
\end{array}\right) .
\end{aligned}
$$

(Both matrices are of the size $L \times L$.) It is easy to see that $\left\|\mathbf{F}_{1}\right\|=\left\|\mathbf{F}_{2}\right\|=1$.

The method called Least Square (briefly, LS) ESPRIT for the analysis of the series $\mathrm{F}$ is based on the following facts (see discussion in [23] and references within): for any basis $U_{1}, \ldots, U_{d}$ of the linear space $\mathbb{U}_{0}^{\perp}$

1. the matrix $\mathbf{U}^{\mathrm{T}} \mathbf{F}_{1} \mathbf{U}$ is invertible;

2. the set of eigenvalues of the matrix

$$
\mathbf{D}=\left(\mathbf{U}^{\mathrm{T}} \mathbf{F}_{1} \mathbf{U}\right)^{-1} \mathbf{U}^{\mathrm{T}} \mathbf{F}_{2} \mathbf{U}
$$

coincides with the set of roots of the polynomial $P_{d}(\lambda)$ subject to multiplicities of roots and eigenvalues.

In practice, the leading left singular vectors of the matrix $\mathbf{H}$ usually stand for the basis $U_{1}, \ldots, U_{d}$ of the linear space $\mathbb{U}_{0}^{\perp}$. Similar choice gives rise to an approximation of the matrix $\mathbf{D}$ in the case when the series $\mathrm{F}$ is corrupted by an additive "noise series" E multiplied by a formal perturbation parameter $\delta$.

Denote $\mathbf{E}$ the $L \times K$ trajectory matrix of the series E. If $U_{j}(\delta)(j=1, \ldots, d)$ are the leading left singular vectors of 
the matrix $\mathbf{H}(\delta)=\mathbf{H}+\delta \mathbf{E}$ and $\mathbf{U}(\delta)=\left[U_{1}(\delta): \ldots: U_{d}(\delta)\right]$, then we can use the matrix

$$
\mathbf{D}(\delta)=\left(\mathbf{U}^{\mathrm{T}}(\delta) \mathbf{F}_{1} \mathbf{U}(\delta)\right)^{-1} \mathbf{U}^{\mathrm{T}}(\delta) \mathbf{F}_{2} \mathbf{U}(\delta)
$$

to approximate $\mathbf{D}$.

The following assertion helps to express $\mathbf{D}(\delta)$ through the perturbed projector $\mathbf{P}_{0}^{\perp}(\delta)$.

Lemma 5.1. Let $U_{1}, \ldots, U_{d}$ be a basis of a linear space $\mathbb{U} \subset \mathbb{R}^{L}$. Denote $\mathbf{P}$ the orthogonal projector on $\mathbb{U}$.

1. If $\mathbf{Q}: \mathbb{R}^{L} \mapsto \mathbb{R}^{L}$ and $\|\mathbf{Q}-\mathbf{P}\|<1$, then vectors $\mathbf{Q} U_{1}, \ldots, \mathbf{Q} U_{d}$ are linearly independent.

2. Consider a linear space $\mathbb{V} \subset \mathbb{R}^{L}$ of dimension $d$ and denote $\mathbf{Q}$ the orthogonal projector on $\mathbb{V}$. If $\|\mathbf{Q}-\mathbf{P}\|<1$, then there exist linearly independent vectors $V_{1}, \ldots, V_{d} \in \mathbb{V}$ such that $U_{j}=\mathbf{P} V_{j}$.

Lemma 5.1 shows that if $\left\|\mathbf{P}_{0}^{\perp}(\delta)-\mathbf{P}_{0}^{\perp}\right\|<1$, then the matrix

$$
\widehat{\mathbf{D}}(\delta)=\left(\mathbf{U}^{\mathrm{T}} \mathbf{P}_{0}^{\perp}(\delta) \mathbf{F}_{1} \mathbf{P}_{0}^{\perp}(\delta) \mathbf{U}\right)^{-1} \mathbf{U}^{\mathrm{T}} \mathbf{P}_{0}^{\perp}(\delta) \mathbf{F}_{2} \mathbf{P}_{0}^{\perp}(\delta) \mathbf{U},
$$

where $\mathbf{U}=\left[U_{1}, \ldots, U_{d}\right]$, has the same eigenvalues as the matrix (5.7) for any choice of the basis $U_{1}, \ldots, U_{d}$ of the linear space $\mathbb{U}_{0}^{\perp}$. Moreover $\widehat{\mathbf{D}}(\delta)=\mathbf{D}(\delta)$ under a certain choice of $U_{1}, \ldots, U_{d}$.

Suppose now that $\left\|\mathbf{P}_{0}^{\perp}(\delta)-\mathbf{P}_{0}^{\perp}\right\| \rightarrow 0$ as $N=K+L-1 \rightarrow \infty$. Then it is natural to suppose that $\|\widehat{\mathbf{D}}(\delta)-\mathbf{D}\| \rightarrow 0$ and therefore asymptotically as $N \rightarrow \infty$ we get all roots of the polynomial $P_{d}(\lambda)$ at least in the case $L=$ const.

Let us formalize these considerations. As earlier, consider a certain basis $U_{1}, \ldots, U_{d}$ of the linear space $\mathbb{U}_{0}^{\perp}$ and set $\mathbf{U}=\left[U_{1}, \ldots, U_{d}\right]$.

Proposition 5.2. If $\Delta \mathbf{P}(\delta) \stackrel{\text { def }}{=}\left\|\mathbf{P}_{0}^{\perp}(\delta)-\mathbf{P}_{0}^{\perp}\right\|<v / 2$ with $v$ standing for $\left\|\mathbf{U}^{\mathrm{T}} \mathbf{F}_{1} \mathbf{U}\right\| /\|\mathbf{U}\|^{2}$, then

$$
\|\widehat{\mathbf{D}}(\delta)-\mathbf{D}\| \leq \frac{2 \Delta \mathbf{P}(\delta)}{v}\left(1+\frac{1}{1-2 \Delta \mathbf{P}(\delta) / v}\right) .
$$

Remark 5.1. Denote $\mathfrak{e}_{L}=(0,0, \ldots, 0,1)^{\mathrm{T}} \in \mathbb{R}^{L}$ and $\vartheta=$ $\left\|\mathbf{P}_{0}^{\perp} \mathfrak{e}_{L}\right\|$. Then $\mathbf{F}_{1}=\mathbf{I}-\mathfrak{e}_{L} \mathfrak{e}_{L}^{\mathrm{T}}$ and

$$
\begin{aligned}
\mathbf{U}^{\mathrm{T}} \mathbf{F}_{1} \mathbf{U} & =\mathbf{U}^{\mathrm{T}} \mathbf{U}-\mathbf{U}^{\mathrm{T}} \mathfrak{e}_{L} \mathfrak{e}_{L}^{\mathrm{T}} \mathbf{U} \\
& =\mathbf{U}^{\mathrm{T}} \mathbf{U}-\mathbf{U}^{\mathrm{T}}\left(\mathbf{P}_{0}^{\perp} \mathfrak{e}_{L}\right)\left(\mathfrak{e}_{L}^{\mathrm{T}} \mathbf{P}_{0}^{\perp}\right) \mathbf{U} .
\end{aligned}
$$

Therefore, $\left\|\mathbf{U}^{\mathrm{T}} \mathbf{F}_{1} \mathbf{U}\right\| \geq\|\mathbf{U}\|^{2}\left(1-\vartheta^{2}\right)$ and under restriction $\Delta \mathbf{P}(\delta)<\left(1-\vartheta^{2}\right) / 2$ the inequality (5.9) can be transformed to

$$
\|\widehat{\mathbf{D}}(\delta)-\mathbf{D}\| \leq \frac{2 \Delta \mathbf{P}(\delta)}{1-\vartheta^{2}}\left(1+\frac{1}{1-2 \Delta \mathbf{P}(\delta) /\left(1-\vartheta^{2}\right)}\right)
$$

Note that the upper bound (5.10) does not depend on a basis of the linear space $\mathbb{U}_{0}^{\perp}$.
The inequality (5.10) shows that $\|\widehat{\mathbf{D}}(\delta)-\mathbf{D}\| \rightarrow 0$ under the same conditions as for the linear recurrent formulas of Section 5.1. More precisely, $\left\|\mathbf{P}_{0}^{\perp}(\delta)-\mathbf{P}_{0}^{\perp}\right\|$ must tend to zero and $\left\|\mathbf{P}_{0} \mathfrak{e}_{L}\right\|^{2}=1-\vartheta^{2}$ must be separated from zero.

Moreover, in this case $\|\widehat{\mathbf{D}}(\delta)-\mathbf{D}\|=O\left(\left\|\mathbf{P}_{0}^{\perp}(\delta)-\mathbf{P}_{0}^{\perp}\right\|\right)$. Therefore, examples of Section 3.2 provide the corresponding upper bounds for $\|\widehat{\mathbf{D}}(\delta)-\mathbf{D}\|$.

\subsection{On the reconstruction stage of SSA}

If the aim of SSA is treated as an interpretable decomposition of time series $\mathrm{G}_{N}=\left(g_{0}, \ldots, g_{N-1}\right)$ onto two or more additive components, then the whole SSA procedure can be expressed as follows. (See [13, Ch. 1] for details; for our goals it is sufficient to decompose $\mathrm{G}_{N}$ onto two components.)

The decomposition stage consists of the choice of the "window length" $L$, the construction of $L \times K$ "trajectory matrix" $\mathbf{G}_{N}$ of the series $\mathrm{G}_{N}$ and the Singular Value Decomposition of $\mathbf{G}$ onto "elementary" rank-one matrices $\mathbf{G}_{N}^{(j)}$.

The reconstruction stage consists of the summation of a certain number of $\mathbf{G}_{N}^{(j)}$ (then we get the "reconstructed" matrix $\mathbf{G}_{N}^{\prime}$ ) and "hankelization" of $\mathbf{G}_{N}^{\prime}$. Formally, the result $\mathcal{S} \mathbf{G}_{N}^{\prime}$ of hankelization of the matrix $\mathbf{G}_{N}^{\prime}=\left\{g_{i j}^{\prime}\right\}_{i=0, j=0}^{L-1, K-1}$ is the $L \times K$ Hankel matrix with elements $\widetilde{g}_{i j}^{\prime}$ equal to the average of $g_{k l}^{\prime}$ such that $k+l=i+j$. Since each $L \times K$ Hankel matrix is in a natural one-to-one correspondence with a series of length $N=L+K-1$, then we obtain the decomposition $\mathrm{G}_{N}=\widetilde{\mathrm{G}}_{N}^{\prime}+\left(\mathrm{G}_{N}-\widetilde{\mathrm{G}}_{N}^{\prime}\right)$ of the initial series $\mathrm{G}_{N}$.

Suppose now that the series $\mathrm{G}_{N}$ is the sum of the "signal" $\mathrm{F}_{N}$ governed by $\operatorname{LRF}(5.1)$ with $b_{d} \neq 0$ and the series $\delta \mathrm{E}_{N}$, where $\mathrm{E}_{N}$ is a "noise series" and $\delta$ is a formal perturbation parameter. Then it is natural to state the problem of an (approximate) extraction of the signal $\mathrm{F}_{N}$ from the sum $\mathrm{G}_{N}=\mathrm{F}_{N}+\delta \mathrm{E}_{N}$.

For small $\delta$ the standard approach to this problem is expressed in terms discussed in previous sections. Namely, under the choice of $d<L<N-d-1$ the trajectory matrix $\mathbf{G}_{N}=\mathbf{H}(\delta)=\mathbf{H}+\delta \mathbf{E}$ is processed by SVD and $d$ leading elementary matrices are summed to get the approximation $\widetilde{\mathbf{H}}=\mathbf{G}_{N}^{\prime}$ of $\mathbf{H}$. Then the hankelization procedure yields the approximation $\widetilde{\mathrm{F}}_{N}(\delta)$ of the series $\mathrm{F}_{N}$.

Note that $\mathbf{H}=\mathbf{P}_{0}^{\perp} \mathbf{H}$. Then $\widetilde{\mathbf{H}}=\mathbf{P}_{0}^{\perp}(\delta)(\mathbf{H}+\delta \mathbf{E})$ and therefore the approximation error $\Delta_{\delta}(\mathbf{H})=\widetilde{\mathbf{H}}-\mathbf{H}$ of the reconstructed trajectory matrix has the form

$$
\begin{aligned}
\Delta_{\delta}(\mathbf{H}) & =\mathbf{P}_{0}^{\perp}(\delta) \mathbf{H}(\delta)-\mathbf{P}_{0}^{\perp} \mathbf{H} \\
& =\left(\mathbf{P}_{0}^{\perp}(\delta)-\mathbf{P}_{0}^{\perp}\right) \mathbf{H}(\delta)+\delta \mathbf{P}_{0}^{\perp} \mathbf{E} .
\end{aligned}
$$

Remark 5.2. It follows from results of Section 2.2.1 that $\widetilde{\mathbf{H}}=\mathbf{H}$ provided that $\mathbf{H}$ and $\mathbf{E}$ are biorthogonal.

Now let us move from (5.11) to the approximation of $\mathrm{F}_{N}$ by the "reconstructed series" $\widetilde{\mathrm{F}}_{N}(\delta)=\left(\widetilde{f}_{0}(\delta), \ldots, \widetilde{f}_{N-1}(\delta)\right)$. 
To measure the difference between $\widetilde{\mathrm{F}}_{N}(\delta)$ and $\mathrm{F}_{N}$ we must introduce a convenient metric. In many practical cases the proper choice is

$$
\left\|\widetilde{\mathrm{F}}_{N}(\delta)-\mathrm{F}_{N}\right\|_{\max }=\max _{0 \leq n<N}\left|\widetilde{f}_{n}(\delta)-f_{n}\right| .
$$

Assume now that for any $N$ both $\mathrm{F}_{N}$ and $\mathrm{E}_{N}$ are segments of infinite series $\mathrm{F}$ and E. If $\left\|\widetilde{\mathrm{F}}_{N}(\delta)-\mathrm{F}_{N}\right\|_{\max }$ tends to zero as $L=L(N)$ and $N \rightarrow \infty$, then SSA asymptotically reconstructs the (infinite) series $\mathrm{F}$ from the (infinite) perturbed series $\mathrm{F}+\delta \mathrm{E}$. It is easy to see that

$$
\begin{aligned}
\left\|\widetilde{\mathrm{F}}_{N}(\delta)-\mathrm{F}_{N}\right\|_{\max } & =\left\|\mathcal{S}\left(\mathbf{P}_{0}^{\perp}(\delta) \mathbf{H}(\delta)\right)-\mathbf{H}\right\|_{\max } \\
& =\left\|\mathcal{S} \Delta_{\delta}(\mathbf{H})\right\|_{\max }
\end{aligned}
$$

where $\|\mathbf{A}\|_{\max }=\max _{i, j}\left|a_{i j}\right|$ for the matrix $\mathbf{A}$ with entries $a_{i j}$ and $\mathcal{S}$ stands for the hankelization operator. It is clear that $\|\mathcal{S} \mathbf{A}\|_{\max } \leq\|\mathbf{A}\|_{\max }$.

The spectral norm $\|\mathbf{A}\|$ and the uniform norm $\|\mathbf{A}\|_{\max }$ are equivalent, but this equivalence is lost if the size of $\mathbf{A}$ tends to infinity.

It is well-known that $\|\mathbf{A}\|_{\max } \leq\|\mathbf{A}\|$. This means that if the spectral norm of the matrix $\mathbf{A}$ is small, then all entries of the Hankel matrix $\mathcal{S} \mathbf{A}$ are small too. The reverse inequality, which is also well-known (see [24] for both inequalities) has the form $\|\mathbf{A}\| \leq \sqrt{L K}\|\mathbf{A}\|_{\max }$ for an $L \times K$ matrix $\mathbf{A}$. This inequality gives a hint that a large-size matrix with small entries can have a large spectral norm. Indeed, the $n \times n$ Hankel matrix $\mathbf{G}_{n}$ with equal entries $g_{i j}^{(n)}=n^{-1 / 2}$ has the spectral norm $\left\|\mathbf{G}_{n}\right\|=\sqrt{n} \rightarrow \infty$ while $g_{i j}^{(n)} \rightarrow 0$ as $n \rightarrow \infty$.

In general, this means that even if the spectral norm $\left\|\Delta_{\delta}(\mathbf{H})\right\|$ does not tend to infinity as $N \rightarrow \infty$, still the convergence $\left\|\widetilde{\mathrm{F}}_{N}(\delta)-\mathrm{F}_{N}\right\|_{\max } \rightarrow 0$ can occur. Therefore, taking into account the last term in the right-hand side of (5.11), we see that general upper bounds of the kind (5.5) or (5.9) can hardly be valid for $\left\|\widetilde{\mathrm{F}}_{N}(\delta)-\mathrm{F}_{N}\right\|_{\max }$.

However, equalities (5.11) and (5.12) help to simplify the problem. Suppose that

$$
\left\|\left(\mathbf{P}_{0}^{\perp}(\delta)-\mathbf{P}_{0}^{\perp}-\mathbf{N}(\delta)\right) \mathbf{H}(\delta)\right\| \rightarrow 0
$$

as $N \rightarrow \infty$ for certain operators $\mathbf{N}(\delta)=\mathbf{N}_{N}(\delta)$. Then

$$
\begin{aligned}
\mathcal{S} \Delta_{\delta}(\mathbf{H})= & \mathcal{S}\left(\left(\mathbf{P}_{0}^{\perp}(\delta)-\mathbf{P}_{0}^{\perp}-\mathbf{N}(\delta)\right) \mathbf{H}(\delta)\right) \\
& +\mathcal{S}\left(\mathbf{N}(\delta) \mathbf{H}(\delta)+\delta \mathbf{P}_{0}^{\perp} \mathbf{E}\right)
\end{aligned}
$$

and

$$
\left\|\widetilde{\mathrm{F}}_{N}(\delta)-\mathrm{F}_{N}\right\|_{\max }=\left\|\mathcal{S}\left(\mathbf{N}(\delta) \mathbf{H}(\delta)+\delta \mathbf{P}_{0}^{\perp} \mathbf{E}\right)\right\|_{\max }+o(1)
$$

as $N \rightarrow \infty$. Thus, the problem reduces to the investigation of the asymptotic behavior of entries of the Hankel matrices $\mathcal{S}\left(\mathbf{N}(\delta) \mathbf{H}(\delta)+\delta \mathbf{P}_{0}^{\perp} \mathbf{E}\right)$.

Let us consider several examples of signal and noise series when the convergence (5.13) occurs. In all examples we straightforwardly apply the inequalities of Section 3.2, therefore the results are given without comments. The norm $\left\|\left(\mathbf{P}_{0}^{\perp}(\delta)-\mathbf{P}_{0}^{\perp}-\mathbf{N}(\delta)\right) \mathbf{H}(\delta)\right\|$ is estimated as

$$
\begin{aligned}
& \left\|\left(\mathbf{P}_{0}^{\perp}(\delta)-\mathbf{P}_{0}^{\perp}-\mathbf{N}(\delta)\right) \mathbf{H}(\delta)\right\| \\
& \quad \leq\left\|\mathbf{P}_{0}^{\perp}(\delta)-\mathbf{P}_{0}^{\perp}-\mathbf{N}(\delta)\right\|(\|\mathbf{H}\|+|\delta|\|\mathbf{E}\|) .
\end{aligned}
$$

In most of examples $\mathbf{N}(\delta)=\delta \mathbf{V}_{0}^{(1)}$. Due to (2.7), we have

$\mathbf{V}_{0}^{(1)} \mathbf{H}(\delta)=\mathbf{P}_{0} \mathbf{E H}^{\mathrm{T}} \mathbf{S}_{0} \mathbf{H}+\delta\left(\mathbf{P}_{0} \mathbf{E} \mathbf{H}^{\mathrm{T}} \mathbf{S}_{0} \mathbf{E}+\mathbf{S}_{0} \mathbf{H} \mathbf{E}^{\mathrm{T}} \mathbf{P}_{0} \mathbf{E}\right)$.

For short, denote

$$
\Lambda(\mathbf{N})=\mathbf{N}(\delta) \mathbf{H}(\delta)+\delta \mathbf{P}_{0}^{\perp} \mathbf{E} \quad \text { and } \quad \lambda(\mathbf{N})=\|\mathcal{S} \Lambda(\mathbf{N})\|_{\max } .
$$

Example 5.1. Signals of exponential type. (See Proposition 3.3 and Table 1 of Section 3.2.1.)

Consider the signal series (3.5) with $\beta_{k} \neq 0$ and decreasing $a_{k}>1$. Then $\|\mathbf{H}\| \asymp a_{1}^{N}$. Denote $\theta=a_{1}^{3 / 2} / a_{p}^{2}$.

1. Let the "noise series" be defined by $e_{n}=\sum_{l=1}^{m} \gamma_{l} b_{l}^{n}$ with $\gamma_{l} \neq 0$ and let $b \stackrel{\text { def }}{=} \max _{1<l<m}\left|b_{l}\right|>1$. Thus $\|\mathbf{E}\| \asymp b^{N}$. If $b \theta<1$, then $\|\mathbf{E}\|=o(\|\mathbf{H}\|)$,

$$
\left\|\mathbf{P}_{0}^{\perp}(\delta)-\mathbf{P}_{0}^{\perp}-\delta \mathbf{V}_{0}^{(1)}\right\|\|\mathbf{H}(\delta)\|=O(b \theta)^{2 N}
$$

for large $N$, and

$$
\left\|\widetilde{\mathrm{F}}_{N}(\delta)-\mathrm{F}_{N}\right\|_{\max }=\lambda\left(\delta \mathbf{V}_{0}^{(1)}\right)+O\left((b \theta)^{2 N}\right) .
$$

2. Consider a polynomial "noise" of order $m$ defined in the manner of (3.6).

a) If $L / N \rightarrow \alpha \in(0,1)$, then $\|\mathbf{E}\| \asymp N^{m+1}=o(\|\mathbf{H}\|)$. If $\theta<1$, then

$$
\left\|\widetilde{\mathrm{F}}_{N}(\delta)-\mathrm{F}_{N}\right\|_{\max }=\lambda\left(\delta \mathbf{V}_{0}^{(1)}\right)+O\left(N^{2 m+2} \theta^{2 N}\right) .
$$

b) If $N \rightarrow \infty$ and either $L$ or $K$ is a constant, then $\|\mathbf{E}\| \asymp N^{m+1 / 2}=o(\|\mathbf{H}\|)$. If $\theta<1$, then

$$
\left\|\widetilde{\mathrm{F}}_{N}(\delta)-\mathrm{F}_{N}\right\|_{\max }=\lambda\left(\delta \mathbf{V}_{0}^{(1)}\right)+O\left(N^{2 m+1} \theta^{2 N}\right) .
$$

3. Let the oscillating "noise series" be defined in the manner of (3.7). Then $\|\mathbf{E}\| \asymp \sqrt{L K}=o(\|\mathbf{H}\|)$. If $\theta<1$, then

$$
\left\|\widetilde{\mathrm{F}}_{N}(\delta)-\mathrm{F}_{N}\right\|_{\max }=\lambda\left(\delta \mathbf{V}_{0}^{(1)}\right)+O\left(L K \theta^{2 N}\right) .
$$

Example 5.2. Oscillating signals. (See Section 3.2.2.) Let the oscillating signal be defined by (3.7). Then $\|\mathbf{H}\| \asymp$ $\sqrt{L K}$. If the "noise series" is also oscillating but with frequencies different from those of a signal, then $\|\mathbf{E}\| \asymp$ $\sqrt{L K} \asymp\|\mathbf{H}\|$ and

$$
\left\|\widetilde{\mathrm{F}}_{N}(\delta)-\mathrm{F}_{N}\right\|_{\max }=\lambda(\mathbf{L}(\delta))+O\left(\sqrt{L K} / \min \left(L^{2}, K^{2}\right)\right)
$$

under the assumption $|\delta|<\delta_{0}$ for some $\delta_{0}>0$. Note that the operator $\mathbf{L}(\delta)$ is defined by (2.25). 
Example 5.3. Random stationary "noise" series. (See Propositions 3.4, 3.5 and Table 2 of Section 3.2.2.)

1. For a signal series of exponential type and under related conditions the following results hold.

a) If $N \rightarrow \infty$ and $L \rightarrow \infty$, then almost surely $\|\mathbf{E}\| / \sqrt{N \ln N}<c$ for $N>N_{0}(\omega)$ and some constant $c>0$. Therefore, $\|\mathbf{E}\|=o(\|\mathbf{H}\|)$ with probability 1 . If $\theta<1$, then

$$
\left\|\widetilde{\mathrm{F}}_{N}(\delta)-\mathrm{F}_{N}\right\|_{\max }=\lambda\left(\delta \mathbf{V}_{0}^{(1)}\right)+O\left(N \ln N \theta^{2 N}\right)
$$

almost surely for $N>N_{0}(\omega, \delta)$.

b) If $L=L_{0}=$ const, then $\|\mathbf{E}\| / \sqrt{N}$ tends a.s. to the positive constant $c_{0}$ and

$$
\left\|\widetilde{\mathrm{F}}_{N}(\delta)-\mathrm{F}_{N}\right\|_{\max }=\lambda\left(\delta \mathbf{V}_{0}^{(1)}\right)+O\left(N \theta^{2 N}\right)
$$

with probability 1 for $N>N_{0}(\omega, \delta)$.

2. Let a polynomial signal of order $p$ be defined by (3.6). If $L / N \rightarrow \alpha \in(0,1)$, then $\|\mathbf{H}\| \asymp N^{p+1}$ while $\|\mathbf{E}\|$ has a.s. the order of growth $\sqrt{N \ln N}$. Therefore,

$$
\left\|\widetilde{\mathrm{F}}_{N}(\delta)-\mathrm{F}_{N}\right\|_{\max }=\lambda\left(\delta \mathbf{V}_{0}^{(1)}\right)+O\left(\ln N / N^{p}\right)
$$

almost surely for $N>N_{0}(\omega, \delta)$.

3. Consider the oscillating signal defined by (3.7) and the white-noise "noise series" as in Proposition 3.5. If $L=$ $L_{0}=$ const, then $\|\mathbf{H}\| \asymp \sqrt{N}$ and $\|\mathbf{E}\| / \sqrt{N} \rightarrow 1$ a.s. Thus $\|\mathbf{H}(\delta)\|=O(\sqrt{N})$ with probability 1 . Since almost surely

$$
\limsup _{N>N_{0}(\omega)} N(\ln \ln N)^{-1}\left\|\mathbf{P}_{0}^{\perp}(\delta)-\mathbf{P}_{0}^{\perp}-\mathbf{T}(\delta)\right\|<c^{\prime \prime} \delta^{2}
$$

with a positive constant $c^{\prime \prime}$, then

$$
\left\|\widetilde{\mathrm{F}}_{N}(\delta)-\mathrm{F}_{N}\right\|_{\max }=\lambda(\mathbf{T}(\delta))+O(\ln \ln N / \sqrt{N})
$$

almost surely for $N>N_{0}(\omega, \delta)$ and $|\delta|<\delta_{0}$.

Let us present two simple examples where these considerations are followed to the end.

\subsubsection{Reconstruction: constant signal and saw "noise series"}

Consider the signal $x_{n}=1$ and the "noise" $e_{n}=(-1)^{n}$. This example was studied in detail in Section 4.2.1, where the main term $\mathbf{M}(\delta)$ of the difference $\mathbf{P}_{0}^{\perp}(\delta)-\mathbf{P}_{0}^{\perp}$ was derived in explicit form, see (4.4), under condition $|\delta|<1 / 2$. Here we use the results and notation of this section.

Proposition 5.3. 1. If both $L$ and $K$ are even, then $\widetilde{\mathrm{F}}_{N}(\delta)=\mathrm{F}_{N}$.

2. If $L$ is even, $K$ is odd, $K \rightarrow \infty$ and $L=o\left(K^{3}\right)$, then

$$
\left\|\widetilde{\mathrm{F}}_{N}(\delta)-\mathrm{F}_{N}\right\|_{\max }=O\left(\max \left(K^{-1}, \sqrt{L / K^{3}}\right)\right) .
$$

3. If $K$ is even, $L$ is odd, $L \rightarrow \infty$ and $K=o\left(L^{3}\right)$, then

$$
\left\|\widetilde{\mathrm{F}}_{N}(\delta)-\mathrm{F}_{N}\right\|_{\max }=O\left(\max \left(L^{-1}, \sqrt{K / L^{3}}\right)\right) .
$$

4. If both $L$ and $K$ are odd, $L=o\left(K^{3}\right)$ and $K=o\left(L^{3}\right)$, then

$$
\left\|\widetilde{\mathrm{F}}_{N}(\delta)-\mathrm{F}_{N}\right\|_{\max }=O\left(\min \left(\sqrt{L / K^{3}}, \sqrt{K / L^{3}}\right)\right) .
$$

Remark 5.3. The best order of convergence to zero of the right-hand side of (5.15) is $N^{-1}$. It is achieved under the choice $L=L_{0}=$ const. The same result for (5.16) is attained by the choice $K=K_{0}$ and for (5.17) by the choice $L \sim N / 2$.

\subsubsection{Reconstruction: exponential signal series and constant "noise series"}

Consider the signal series $x_{n}=a^{n}$ with $a>1$ and the "noise series" $e_{n} \equiv 1$. Then $\theta=\sqrt{a}$ and applying (5.14) we see that

$$
\left\|\widetilde{\mathrm{F}}_{N}(\delta)-\mathrm{F}_{N}\right\|_{\max }=\lambda\left(\delta \mathbf{V}_{0}^{(1)}\right)+O\left(N^{2} a^{-N}\right)
$$

in the case $L / N \rightarrow \alpha \in(0,1)$ and that

$$
\left\|\widetilde{\mathrm{F}}_{N}(\delta)-\mathrm{F}_{N}\right\|_{\max }=\lambda\left(\delta \mathbf{V}_{0}^{(1)}\right)+O\left(N a^{-N}\right)
$$

in the case when $N \rightarrow \infty$ and either $L$ or $K$ is a constant.

Proposition 5.4. If $L / N \rightarrow \alpha \in(0,1)$ or if $N \rightarrow \infty$ and either $L$ or $K$ is a constant, then

$$
\underset{N}{\limsup }\left\|\widetilde{\mathrm{F}}_{N}(\delta)-\mathrm{F}_{N}\right\|_{\max }>0 .
$$

The accurate analysis of the matrix $\mathcal{S} \Lambda_{1}\left(\delta \mathbf{V}_{0}^{(1)}\right)$ gives much more information on the asymptotic behavior of the series $\widetilde{\mathrm{F}}_{N}(\delta)$. For example, in the case of odd $N$ and $L=K$ it can be proved that $\max _{0 \leq l<(1-\varepsilon) N}\left|\widetilde{f}_{l}(\delta)-f_{l}\right| \rightarrow 0$ as $N \rightarrow \infty$ for any small $\varepsilon>0$, see details in [21, Sect. 5.3.2].

\section{APPENDIX: PROOFS}

\section{Proof of Theorem 2.2}

1. The equivalence between (2.10) and (2.11). Since

$$
\mathbf{S}_{0} \mathbf{B}(\delta) \mathbf{P}_{0}=\delta \mathbf{S}_{0} \mathbf{H E}^{\mathrm{T}} \mathbf{P}_{0}+\delta^{2} \mathbf{S}_{0} \mathbf{E E}^{\mathrm{T}} \mathbf{P}_{0}
$$

then (2.11) automatically follows from (2.10). On the other hand, if $\mathbf{S}_{0} \mathbf{H} \mathbf{E}^{\mathrm{T}} \mathbf{P}_{0}+\delta \mathbf{S}_{0} \mathbf{E} \mathbf{E}^{\mathrm{T}} \mathbf{P}_{0}=\mathbf{0}_{L L}$ for all $\delta \in(-\varepsilon, \varepsilon)$, then we obtain the equalities (2.10).

2. The equivalence between (2.10) and (2.12). It is easy to see that (2.12) follows from (2.10). Let us demonstrate that the equality $\mathbf{S}_{0} \mathbf{H E}^{\mathrm{T}} \mathbf{P}_{0}=\mathbf{0}_{L L}$ follows from the equality

$$
\mathbf{S}_{0} \mathbf{H} \mathbf{E}^{\mathrm{T}} \mathbf{P}_{0}+\mathbf{P}_{0} \mathbf{H} \mathbf{E}^{\mathrm{T}} \mathbf{S}_{0}=\mathbf{0}_{L L}
$$

and that the equality $\mathbf{S}_{0} \mathbf{E} \mathbf{E}^{\mathrm{T}} \mathbf{P}_{0}=\mathbf{0}_{L L}$ is a consequence of $\mathbf{S}_{0} \mathbf{E E}^{\mathrm{T}} \mathbf{P}_{0}+\mathbf{P}_{0} \mathbf{E} \mathbf{E}^{\mathrm{T}} \mathbf{S}_{0}=\mathbf{0}_{L L}$. Both assertions have similar proofs. Suppose that (6.1) holds and denote $X=\mathbf{H E}^{\mathrm{T}} \mathbf{P}_{0} Z$ 
and $Y=\mathbf{H E}^{\mathrm{T}} \mathbf{S}_{0} Z$ for some $Z \in \mathbb{R}^{L}$. Then the equality $\mathbf{S}_{0} X+\mathbf{P}_{0} Y=0_{L}$ follows from (6.1). Since $\mathbf{S}_{0} X \in \mathbb{U}_{0}^{\perp}$ and $\mathbf{P}_{0} Y \in \mathbb{U}_{0}$, the latter vectors are orthogonal. Thus $\mathbf{S}_{0} \mathbf{H E}^{\mathrm{T}} \mathbf{P}_{0} Z=\mathbf{P}_{0} \mathbf{H E}^{\mathrm{T}} \mathbf{S}_{0} Z=0_{L}$ for all $Z \in \mathbb{R}^{L}$.

3. The equivalence between the equality $\mathbf{P}_{0}^{\perp}(\delta)=\mathbf{P}_{0}^{\perp}$ and (2.10). Assume that (2.10) holds. Then $\mathbf{S}_{0} \mathbf{B}(\delta) \mathbf{P}_{0}=$ $\mathbf{P}_{0} \mathbf{B}(\delta) \mathbf{S}_{0}=\mathbf{0}_{L L}$. Consider the operator

$$
\mathbf{S}_{0}^{\left(l_{1}\right)} \mathbf{B}(\delta) \mathbf{S}_{0}^{\left(l_{2}\right)} \ldots \mathbf{S}_{0}^{\left(l_{p}\right)} \mathbf{B}(\delta) \mathbf{S}_{0}^{\left(l_{p+1}\right)}
$$

see (2.3). Since $l_{1}+\cdots+l_{p+1}=p$, then there exists $j$ such that $l_{j}>0$ and either $l_{j-1}=0$ or $l_{j+1}=0$. Suppose that $l_{j+1}=0$. (The case $l_{j-1}=0$ is studied analogously.) Since $\mathbf{S}_{0}^{(0)}=-\mathbf{P}_{0}$ and $\mathbf{S}_{0}^{\left(l_{j}\right)}=\mathbf{S}^{l_{j}}$, then $\mathbf{S}_{0}^{\left(l_{j}\right)} \mathbf{B}(\delta) \mathbf{S}_{0}^{\left(l_{j+1}\right)}=\mathbf{0}$ and the operator (6.2) is the zero operator. Hence $\mathbf{W}_{p}(\delta)=\mathbf{0}_{L L}$ for all $p$, and $\mathbf{P}_{0}^{\perp}(\delta)=\mathbf{P}_{0}^{\perp}$.

In order to prove the converse assertion we consider the expansion (2.4). Since $\mathbf{P}_{0}^{\perp}(\delta)=\mathbf{P}_{0}^{\perp}$ for all $\delta \in\left(-\delta_{0}, \delta_{0}\right)$, then the coefficients $\mathbf{V}_{0}^{(n)}$ of the power series (2.4) are zero operators. In particular, $\mathbf{V}_{0}^{(1)}=\mathbf{0}_{L L}$ and (6.1) holds due to (2.7). Let us consider the coefficient $\mathbf{V}_{0}^{(2)}$. As was already proved, $\mathbf{S}_{0} \mathbf{A}^{(1)} \mathbf{P}_{0}=\mathbf{P}_{0} \mathbf{A}^{(1)} \mathbf{S}_{0}=\mathbf{0}_{L L}$. Thus (2.8) shows that $\mathbf{0}_{L L}=\mathbf{V}_{0}^{(2)}=\mathbf{P}_{0} \mathbf{A}^{(2)} \mathbf{S}_{0}+\mathbf{S}_{0} \mathbf{A}^{(2)} \mathbf{P}_{0}$, and we get (2.12). The latter equality is equivalent to (2.10).

4. The equivalence between (2.10) and (2.13). Let us prove that the equality $\mathbf{H E}^{\mathrm{T}} \mathbf{P}_{0}=\mathbf{0}_{L L}$ follows from $\mathbf{S}_{0} \mathbf{H E}^{\mathrm{T}} \mathbf{P}_{0}=\mathbf{0}_{L L}$. (The converse statement holds automatically.) Suppose that $\mathbf{S}_{0} \mathbf{H E}^{\mathrm{T}} \mathbf{P}_{0}=\mathbf{0}_{L L}$ and let $Z \in \mathbb{R}^{L}$. Note that vectors $\mathbf{P}_{\mu} \mathbf{H E}^{\mathrm{T}} \mathbf{P}_{0} Z$ are pairwise orthogonal for different $\mu \in \Sigma$. Therefore, since

$$
\mathbf{S}_{0} \mathbf{H E}^{\mathrm{T}} \mathbf{P}_{0} Z=\sum_{\mu>0} \mu^{-1} \mathbf{P}_{\mu} \mathbf{H E}^{\mathrm{T}} \mathbf{P}_{0} Z=0_{L},
$$

then $\mathbf{P}_{\mu} \mathbf{H} \mathbf{E}^{\mathrm{T}} \mathbf{P}_{0} Z=0_{L}$ for all non-zero $\mu \in \Sigma$. In view of the equality $\mathbf{H}=\sum_{\mu>0} \mathbf{P}_{\mu} \mathbf{H}$ we see that $\mathbf{H} \mathbf{E}^{\mathrm{T}} \mathbf{P}_{0}=\mathbf{0}$.

Let us prove that the equality $\mathbf{H}^{\mathrm{T}} \mathbf{E} \mathbf{E}^{\mathrm{T}} \mathbf{P}_{0}=\mathbf{0}_{L K}$ holds iff $\mathbf{S}_{0} \mathbf{E E}^{\mathrm{T}} \mathbf{P}_{0}=\mathbf{0}_{L L}$. If $\mathbf{H}^{\mathrm{T}} \mathbf{E} \mathbf{E}^{\mathrm{T}} \mathbf{P}_{0}=\mathbf{0}_{L K}$, then

$$
\mathbf{0}_{L L}=\mathbf{H} \mathbf{H}^{\mathrm{T}} \mathbf{E} \mathbf{E}^{\mathrm{T}} \mathbf{P}_{0}=\sum_{\mu>0} \mu \mathbf{P}_{\mu} \mathbf{E E}^{\mathrm{T}} \mathbf{P}_{0}
$$

$\mathbf{P}_{\mu} \mathbf{E E}^{\mathrm{T}} \mathbf{P}_{0}=\mathbf{0}_{L L}$ for all non-zero $\mu \in \Sigma$, and $\mathbf{S}_{0} \mathbf{E E}^{\mathrm{T}} \mathbf{P}_{0}=$ $\mathbf{0}_{L L}$. The converse statement is proved in the same manner due to the equality $\mathbf{H}^{\mathrm{T}} \mathbf{E} \mathbf{E}^{\mathrm{T}} \mathbf{P}_{0}=\sum_{\mu>0} \mathbf{H}^{\mathrm{T}} \mathbf{P}_{\mu} \mathbf{E E}^{\mathrm{T}} \mathbf{P}_{0}$. This completes the entire proof.

\section{Proof of Lemma 2.1}

1. Let $\mathbf{H}^{\mathrm{T}} \mathbf{E}=\mathbf{0}_{K K}$. Then $\mathbf{H}^{\mathrm{T}} \mathbf{H E} X=0_{L}$ for any $X \in$ $\mathbb{R}^{K}$. If $0 \notin \Sigma$, then $\mathbf{E} X=0_{L}$ and $\mathbf{E}=\mathbf{0}_{K L}$. Therefore, $0 \in \Sigma$ and $\mathbf{E} X \in \mathbb{U}_{0}$. Thus $\mathbf{P}_{0} \mathbf{E} X=\mathbf{E} X$ and $\mathbf{P}_{0} \mathbf{E}=\mathbf{E}$. Analogously, $\mathbf{P}_{\mu} \mathbf{E}=\mathbf{P}_{\mu} \mathbf{P}_{0} \mathbf{E}=\mathbf{0}_{K L}$ for all non-zero $\mu \in \Sigma$.

2. Since $\mathbf{H}=\sum_{\mu>0} \mathbf{P}_{\mu} \mathbf{H}$, then $\mathbf{H}^{\mathrm{T}} \mathbf{E}=\mathbf{H}^{\mathrm{T}}\left(\sum_{\mu>0} \mathbf{P}_{\mu} \mathbf{E}\right)$, and the second assertion is proved as well.

\section{Proof of Theorem 2.3}

Let us start with the following lemma.

Lemma 6.1. If $0<\beta<1 / 4$ and $k \geq 0$, then

$$
\sum_{p=k}^{\infty}\left(\begin{array}{c}
2 p \\
p
\end{array}\right) \beta^{p} \leq C \frac{(4 \beta)^{k}}{1-4 \beta}
$$

where $C=e^{1 / 6} / \sqrt{\pi} \approx 0.667$.

Proof. Since $n !=\sqrt{2 \pi} n^{n+1 / 2} e^{-n} e^{\theta_{n} / 12 n}$ with $0<\theta_{n}<1$, then

$$
\left(\begin{array}{c}
2 p \\
p
\end{array}\right)=\frac{1}{\sqrt{2 \pi}} \frac{\sqrt{2 p}}{p} \frac{4^{p} p^{2 p}}{p^{2 p}} e^{\left(\theta_{2 p}-\theta_{p}\right) / 6 p} \leq \frac{1}{\sqrt{\pi p}} 4^{p} e^{1 / 6 p} \leq C 4^{p},
$$

and the result becomes clear.

Now let us demonstrate Theorem 2.3. Due to Theorem 2.1, we can use the expansion (2.2).

In the same way as in the demonstration of Theorem 2.2, consider the term (6.2) and take $j$ such that $l_{j}>0$ and $l_{j+1}=0$ (the case $l_{j-1}=0$ is quite analogous). Since $l_{1}+$ $\cdots+l_{p+1}=p$ and $\left\|\mathbf{S}_{0}^{(k)}\right\|=1 / \mu_{\min }^{k}$ for any $k \geq 0$, then the norm of the operator (6.2) can be estimated from above:

$$
\begin{aligned}
& \left\|\mathbf{S}_{0}^{\left(l_{1}\right)} \mathbf{B}(\delta) \mathbf{S}_{0}^{\left(l_{2}\right)} \ldots \mathbf{B}(\delta) \mathbf{S}_{0}^{\left(l_{p+1}\right)}\right\| \\
& \quad=\left\|\mathbf{S}_{0}^{\left(l_{1}\right)} \mathbf{B}(\delta) \ldots \mathbf{S}_{0}^{\left(l_{j}-1\right)} \mathbf{S}_{0} \mathbf{B}(\delta) \mathbf{P}_{0} \ldots \mathbf{B}(\delta) \mathbf{S}_{0}^{\left(l_{p+1}\right)}\right\| \\
& \leq\left\|\mathbf{S}_{0} \mathbf{B}(\delta) \mathbf{P}_{0}\right\|\left(\|\mathbf{B}(\delta)\| / \mu_{\min }\right)^{p-1} .
\end{aligned}
$$

Denote $\Im_{p}$ the number of nonnegative integer vectors $\left(l_{1}, \ldots, l_{p+1}\right)$ such that $l_{1}+\cdots+l_{p+1}=p$. Then $\Im_{p}=\left(\begin{array}{c}2 p \\ p\end{array}\right)$. In view of (2.2) and (6.4),

$$
\begin{aligned}
& \left\|\mathbf{P}_{0}^{\perp}(\delta)-\mathbf{P}_{0}^{\perp}\right\| \\
& \leq\left\|\mathbf{S}_{0} \mathbf{B}(\delta) \mathbf{P}_{0}\right\| \sum_{p=1}^{\infty} \sum_{l_{1}+\cdots+l_{p+1}=p, l_{j} \geq 0}\left(\|\mathbf{B}(\delta)\| / \mu_{\text {min }}\right)^{p-1} \\
& =\left\|\mathbf{S}_{0} \mathbf{B}(\delta) \mathbf{P}_{0}\right\| \sum_{p=1}^{\infty}\left(\begin{array}{c}
2 p \\
p
\end{array}\right)\left(\|\mathbf{B}(\delta)\| / \mu_{\text {min }}\right)^{p-1} .
\end{aligned}
$$

Now we get the result with the help of (6.3).

\section{Proof of Corollary 2.1}

Since $\left\|\mathbf{S}_{0} \mathbf{B}(\delta) \mathbf{P}_{0}\right\| \leq\|\mathbf{B}(\delta)\| / \mu_{\min }$, then (2.16) automatically follows from (2.15). The inequality $\|\mathbf{B}(\delta)\| / \mu_{\min } \leq$ $B(\delta) / \mu_{\min }$ was already established. The chain of inequalities

$$
\begin{aligned}
\frac{\|\mathbf{B}(\delta)\|}{\mu_{\min }} & \leq \frac{B(\delta)}{\mu_{\min }} \leq 2|\delta| \frac{\left\|\mathbf{H E}^{\mathrm{T}}\right\|}{\mu_{\min }}+\delta^{2} \frac{\nu_{\max }}{\mu_{\min }} \\
& \leq 2|\delta|\|\mathbf{H}\|\|\mathbf{E}\| / \mu_{\min }+\delta^{2} \Theta_{1}^{2} \Theta_{2} \\
& =2|\delta| \Theta_{1} \Theta_{2}+\delta^{2} \Theta_{1}^{2} \Theta_{2}
\end{aligned}
$$

provides the result. 


\section{Proof of Proposition 2.1}

We begin with the following lemma.

Lemma 6.2. Consider the matrix $\mathbf{M}: \mathbb{R}^{K} \mapsto \mathbb{R}^{L}$ and let $\mathbb{U}_{M}$ stand for the linear space spanned by columns of the matrix $\mathbf{M}$. Denote $\sigma_{\min }$ and $\sigma_{\max }$ the minimal and maximal singular values of $\mathbf{M}$. Then

$$
\left\{\mathbf{M} X,\|X\| \leq 1 / \sigma_{\max }\right\} \subset\left\{Y \in \mathbb{U}_{M},\|Y\| \leq 1\right\}
$$

and

$$
\left\{\mathbf{M} X,\|X\| \leq 1 / \sigma_{\min }\right\} \supset\left\{Y \in \mathbb{U}_{M},\|Y\| \leq 1\right\} .
$$

Proof. The inclusion (6.5) straightforwardly follows from the inequality $\|\mathbf{M} X\| \leq\|\mathbf{M}\|\|X\| \leq 1$. Let us prove the inclusion (6.6).

Consider the SVD of the matrix $\mathbf{M}: \mathbf{M}=\sum_{j} \sigma_{j} U_{j} V_{j}^{\mathrm{T}}$. By the definition, the left singular vectors $U_{j}$ form the orthonormal basis of $\mathbb{U}_{M}$. (Analogously, the right singular vectors $V_{j}$ form the orthonormal basis of the linear space spanned by rows of the same matrix.)

Let us prove that for any $Y \in \mathbb{U}_{M}$ with $\|Y\| \leq 1$ there exists $X \in \mathbb{R}^{K}$ such that $\|X\| \leq 1 / \sigma_{\min }$ and $\mathbf{M} X=Y$. Then (6.6) will be proved as well.

Note that $Y=\sum_{j} c_{j} U_{j}$ with $\sum_{j} c_{j}^{2} \leq 1$. In the same manner, each $X \in \mathbb{R}^{K}$ can be expressed as $X=\sum_{j} \alpha_{j} V_{j}+$ $\beta_{0} W_{0}$, where $<V_{j}, W_{0}>_{K}=0$ and $<,>_{K}$ stands for the inner product in $\mathbb{R}^{K}$. Therefore, $\mathbf{M} X=\sum_{j} \sigma_{j} \alpha_{j} U_{j}$. If we set $\alpha_{j}=c_{j} / \sigma_{j}$, then $\sum_{j} \alpha_{j}^{2} \leq \sum_{j} c_{j}^{2} / \sigma_{\min }^{2} \leq 1 / \sigma_{\min }^{2}$ and the proof is complete.

Let us now turn to the proof of Proposition 2.1. By the definition,

$$
\cos \left(\theta_{\min }\right)=\max _{Y_{1} \in \mathbb{U}_{1},\left\|Y_{1}\right\| \leq 1} \max _{Y_{2} \in \mathbb{U}_{2},\left\|Y_{2}\right\| \leq 1}\left\langle Y_{1}, Y_{2}\right\rangle_{L} .
$$

In view of Lemma 6.2,

$$
\begin{aligned}
& \left\|\mathbf{M}_{1}\right\|\left\|\mathbf{M}_{2}\right\| \cos \left(\theta_{\min }\right) \\
& \leq \max _{X_{1} \in \mathbb{R}^{K},\left\|X_{1}\right\| \leq 1} \max _{X_{2} \in \mathbb{R}^{K},\left\|X_{2}\right\| \leq 1}\left\langle\mathbf{M}_{1} X_{1}, \mathbf{M}_{2} X_{2}\right\rangle_{L} \\
& =\max _{X_{1} \in \mathbb{R}^{K},\left\|X_{1}\right\| \leq 1} \max _{X_{2} \in \mathbb{R}^{K},\left\|X_{2}\right\| \leq 1}\left\langle\mathbf{M}_{1}^{\mathrm{T}} \mathbf{M}_{2} X_{1}, X_{2}\right\rangle_{K} \\
& =\left\|\mathbf{M}_{1}^{\mathrm{T}} \mathbf{M}_{2}\right\| .
\end{aligned}
$$

If we use (6.6) instead of (6.5), then we get

$$
\begin{aligned}
& \sigma_{1}^{(\min )} \sigma_{2}^{(\min )} \cos \left(\theta_{\min }\right) \\
& \quad \geq \max _{X_{1} \in \mathbb{R}^{K},\left\|X_{1}\right\| \leq 1} \max _{X_{2} \in \mathbb{R}^{K},\left\|X_{2}\right\| \leq 1}\left\langle\mathbf{M}_{1} X_{1}, \mathbf{M}_{2} X_{2}\right\rangle_{L} \\
& =\left\|\mathbf{M}_{1}^{\mathrm{T}} \mathbf{M}_{2}\right\| .
\end{aligned}
$$

This completes the proof.

\section{Proof of Corollary 2.2}

It is easy to see that (2.20) follows from (2.15). Besides,

$$
\left\|\mathbf{S}_{0} \mathbf{B}(\delta)\right\| \leq 2|\delta|\left\|\mathbf{H} \mathbf{E}^{\mathrm{T}}\right\| / \mu_{\min }+\delta^{2}\left\|\mathbf{S}_{0} \mathbf{A}^{(2)}\right\| .
$$

Proposition 2.1 shows that

$$
\left\|\mathbf{H E}^{\mathrm{T}}\right\| / \mu_{\min } \leq \cos \left(\theta_{r}\right) \sqrt{\mu_{\max } \nu_{\max }} / \mu_{\min }=\Theta_{1} \Theta_{2} \cos \left(\theta_{r}\right) .
$$

As for the term $\left\|\mathbf{S}_{0} \mathbf{A}^{(2)}\right\|$, it is worth mentioning that the columns of the matrix $\mathbf{S}_{0}=\mathbf{S}_{0}^{\mathrm{T}}$ span the same linear space $\mathbb{U}_{0}^{\perp}$ as the columns of the matrix $\mathbf{H}$. Analogously, the columns of $\mathbf{A}^{(2)}$ span the same linear space as the columns of $\mathbf{E}$. Therefore, it follows from Proposition 2.1 that $\left\|\mathbf{S}_{0} \mathbf{A}^{(2)}\right\| \leq \cos \left(\theta_{l}\right) \nu_{\max } / \mu_{\min }=\Theta_{1}^{2} \Theta_{2} \cos \left(\theta_{l}\right)$, and the proof is complete.

\section{Proof of Theorem 2.4}

Acting in the same manner as in Theorem 2.3 and taking into consideration that $\left\|\mathbf{S}_{0} \mathbf{B}(\delta)\right\| \leq\|\mathbf{B}(\delta)\| / \mu_{\text {min }}$ we obtain the inequality

$$
\left\|\mathbf{P}_{0}^{\perp}(\delta)-\mathbf{P}_{0}^{\perp}-\mathbf{W}_{1}(\delta)\right\| \leq \sum_{p=2}^{\infty}\left(\begin{array}{c}
2 p \\
p
\end{array}\right)\left(\frac{\|\mathbf{B}(\delta)\|}{\mu_{\min }}\right)^{p} .
$$

Applying Lemma 6.1 and the condition $\|\mathbf{B}(\delta)\|<\mu_{\min } / 4$ we obtain (2.22).

\section{Proof of Theorem 2.5}

First of all, we can use the decomposition (2.2). Let us extract the operator $\mathbf{L}(\delta)$ from the right-hand side of (2.2).

For fixed $p \geq 1$ we take the vector $\left(l_{1}, \ldots, l_{p+1}\right)$ with $l_{0}=p$ and $l_{j}=0$ for $j \geq 1$ and consider the related operator

$$
\mathbf{X}_{1}(p)=(-1)^{p} \mathbf{S}_{0}^{\left(l_{1}\right)} \mathbf{B}(\delta) \mathbf{S}_{0}^{\left(l_{2}\right)} \ldots \mathbf{B}(\delta) \mathbf{S}_{0}^{\left(l_{p+1}\right)}
$$

It is clear that

$$
\begin{aligned}
\mathbf{X}_{1}(p) & =(-1)^{p} \mathbf{S}_{0}^{(p)} \mathbf{B}(\delta) \mathbf{S}_{0}^{(0)} \ldots \mathbf{B}(\delta) \mathbf{S}_{0}^{(0)} \\
& =\mathbf{S}_{0}^{p} \mathbf{B}(\delta) \mathbf{P}_{0} \ldots \mathbf{B}(\delta) \mathbf{P}_{0} \\
& =\mathbf{S}_{0}^{p} \mathbf{B}(\delta) \mathbf{P}_{0}\left(\mathbf{P}_{0} \mathbf{B}(\delta) \mathbf{P}_{0}\right)^{p-1}
\end{aligned}
$$

Since $\mathbf{P}_{0} \mathbf{B}(\delta) \mathbf{P}_{0}=\delta^{2} \mathbf{A}_{0}^{(2)}$ and $\mathbf{S}_{0}^{p}=\sum_{\mu>0} \mathbf{P}_{\mu} / \mu^{p}$, then

$$
\begin{aligned}
\mathbf{X}_{1}(p) & =\sum_{\mu>0} \mu^{-p} \mathbf{P}_{\mu} \mathbf{B}(\delta) \mathbf{P}_{0}\left(\delta^{2} \mathbf{A}_{0}^{(2)}\right)^{p-1} \\
& =\sum_{\mu>0} \mu^{-1} \mathbf{P}_{\mu} \mathbf{B}(\delta) \mathbf{P}_{0}\left(\delta^{2} \mathbf{A}_{0}^{(2)} / \mu\right)^{p-1} .
\end{aligned}
$$

Thus we obtain that

$$
\begin{aligned}
\sum_{p=1}^{\infty} \mathbf{X}_{1}(p) & =\sum_{\mu>0} \mu^{-1} \mathbf{P}_{\mu} \mathbf{B}(\delta) \mathbf{P}_{0} \sum_{p=1}^{\infty}\left(\delta^{2} \mathbf{A}_{0}^{(2)} / \mu\right)^{p-1} \\
& =\sum_{\mu>0} \mu^{-1} \mathbf{P}_{\mu} \mathbf{B}(\delta) \mathbf{P}_{0}\left(\mathbf{I}-\delta^{2} \mathbf{A}_{0}^{(2)} / \mu\right)^{-1} .
\end{aligned}
$$


If we consider $\mathbf{X}_{2}(p)=(-1)^{p} \mathbf{S}_{0}^{(0)} \mathbf{B}(\delta) \mathbf{S}_{0}^{(0)} \ldots \mathbf{B}(\delta) \mathbf{S}_{0}^{(p)}$ and act in the same manner as for $\mathbf{X}_{1}(p)$, we get

$$
\sum_{p=1}^{\infty} \mathbf{X}_{2}(p)=\sum_{\mu>0}\left(\mathbf{I}-\delta^{2} \mathbf{A}_{0}^{(2)} / \mu\right)^{-1} \mathbf{P}_{0} \mathbf{B}(\delta) \mathbf{P}_{\mu} / \mu
$$

Thus $\mathbf{L}(p)=\sum_{p \geq 1}\left(\mathbf{X}_{1}(p)+\mathbf{X}_{2}(p)\right)$.

Now we consider the vector $\left(l_{1}, \ldots, l_{p+1}\right)$ such that $\sum_{j=1}^{p+1} l_{j}=p$ and neither $l_{1}$ nor $l_{p+1}$ equals $p$. (It follows from this condition that $p>1$.) In addition, consider $1 \leq j \leq p+1$ such that $l_{j}>0$ and either $l_{j-1}$ or $l_{j+1}$ equals zero. If $l_{j}=p$ and $1<j<p+1$, then

$$
\begin{aligned}
& \left\|\mathbf{S}_{0}^{(0)} \mathbf{B}(\delta) \ldots \mathbf{B}(\delta) \mathbf{S}_{0}^{(p)} \mathbf{B}(\delta) \ldots \mathbf{B}(\delta) \mathbf{S}_{0}^{(0)}\right\| \\
& \quad=\left\|\mathbf{P}_{0} \mathbf{B}(\delta) \ldots \mathbf{P}_{0} \mathbf{B}(\delta) \mathbf{S}_{0} \mathbf{S}_{0}^{p-2} \mathbf{S}_{0} \mathbf{B}(\delta) \mathbf{P}_{0} \ldots \mathbf{B}(\delta) \mathbf{P}_{0}\right\| \\
& \leq\left\|\mathbf{P}_{0} \mathbf{B}(\delta) \mathbf{S}_{0}\right\|\left\|\mathbf{S}_{0} \mathbf{B}(\delta) \mathbf{P}_{0}\right\|\left\|\mathbf{S}_{0}^{p-2}\right\|\|\mathbf{B}(\delta)\|^{p-2} \\
& \leq\left\|\mathbf{S}_{0} \mathbf{B}(\delta) \mathbf{P}_{0}\right\|\left\|\mathbf{S}_{0} \mathbf{B}(\delta)\right\|\left(\|\mathbf{B}(\delta)\| / \mu_{\min }\right)^{p-2}
\end{aligned}
$$

If $l_{j} \neq p$, then there exists $k \neq j$ such that $l_{k} \neq 0$ and we get the same upper bound (6.8). For example, let $1<l_{k}<$ $l_{j}<p+1$ and $l_{j+1}=0$. Then

$$
\begin{aligned}
& \left\|\mathbf{S}_{0}^{\left(l_{1}\right)} \mathbf{B}(\delta) \ldots \mathbf{B}(\delta) \mathbf{S}_{0}^{\left(l_{k}\right)} \ldots \mathbf{S}_{0}^{\left(l_{j}\right)} \mathbf{B}(\delta) \ldots \mathbf{B}(\delta) \mathbf{S}_{0}^{\left(l_{p+1}\right)}\right\| \\
& \quad=\left\|\mathbf{S}_{0}^{\left(l_{1}\right)} \ldots \mathbf{B}(\delta) \mathbf{S}_{0} \mathbf{S}_{0}^{\left(l_{k}-1\right)} \ldots \mathbf{S}_{0}^{\left(l_{j}-1\right)} \mathbf{S}_{0} \mathbf{B}(\delta) \mathbf{P}_{0} \ldots \mathbf{S}_{0}^{\left(l_{p+1}\right)}\right\| \\
& \leq\left\|\mathbf{S}_{0} \mathbf{B}(\delta)\right\|\left\|\mathbf{S}_{0} \mathbf{B}(\delta) \mathbf{P}_{0}\right\|\|\mathbf{B}\|^{p-2}\left\|\mathbf{S}_{0}\right\|^{l_{1}+\cdots+l_{p+1}-2} \\
& =\left\|\mathbf{S}_{0} \mathbf{B}(\delta)\right\|\left\|\mathbf{S}_{0} \mathbf{B}(\delta) \mathbf{P}_{0}\right\|\left(\|\mathbf{B}(\delta)\| / \mu_{\min }\right)^{p-2} .
\end{aligned}
$$

Other variants concerning $k, j$ provide the same result. Therefore, applying (2.2) and Lemma 6.1 we obtain that

$$
\begin{aligned}
& \left\|\mathbf{P}_{0}^{\perp}(\delta)-\mathbf{P}_{0}^{\perp}-\mathbf{L}(\delta)\right\| \\
& =\left\|\sum_{p=2}^{\infty}(-1)^{p} \sum_{\substack{l_{1}+\cdots+l_{p+1}=p \\
l_{j} \geq 0, l_{1} \neq p, l_{p+1} \neq p}} \mathbf{S}_{0}^{\left(l_{1}\right)} \mathbf{B}(\delta) \mathbf{S}_{0}^{\left(l_{2}\right)} \ldots \mathbf{B}(\delta) \mathbf{S}_{0}^{\left(l_{p+1}\right)}\right\| \\
& \leq \sum_{p=2}^{\infty} \sum_{\substack{l_{1}+\cdots+l_{p+1}=p \\
l_{j} \geq 0, l_{1} \neq p, l_{p+1} \neq p}}\left\|\mathbf{S}_{0} \mathbf{B}(\delta)\right\|\left\|\mathbf{S}_{0} \mathbf{B}(\delta) \mathbf{P}_{0}\right\|\left(\frac{\|\mathbf{B}(\delta)\|}{\mu_{\min }}\right)^{p-2} \\
& =\left\|\mathbf{S}_{0} \mathbf{B}(\delta)\right\|\left\|\mathbf{S}_{0} \mathbf{B}(\delta) \mathbf{P}_{0}\right\| \sum_{p=2}^{\infty}\left(\left(\begin{array}{c}
2 p \\
p
\end{array}\right)-2\right)\left(\frac{\|\mathbf{B}(\delta)\|}{\mu_{\min }}\right)^{p-2} \\
& <\left\|\mathbf{S}_{0} \mathbf{B}(\delta)\right\|\left\|\mathbf{S}_{0} \mathbf{B}(\delta) \mathbf{P}_{0}\right\| \sum_{p=2}^{\infty}\left(\begin{array}{c}
2 p \\
p
\end{array}\right)\left(\frac{\|\mathbf{B}(\delta)\|}{\mu_{\min }}\right)^{p-2} \\
& \leq 16 C \frac{\left\|\mathbf{S}_{0} \mathbf{B}(\delta)\right\|\left\|\mathbf{S}_{0} \mathbf{B}(\delta) \mathbf{P}_{0}\right\|}{1-4\|\mathbf{B}(\delta)\| / \mu_{\min }} .
\end{aligned}
$$

The proof is complete.

\section{Proof of Proposition 2.2}

First of all we have

$$
\left(\mathbf{I}-\delta^{2} \mathbf{A}_{0}^{(2)} / \mu\right)^{-1}-\mathbf{I}=\left(\mathbf{I}-\delta^{2} \mathbf{A}_{0}^{(2)} / \mu\right)^{-1} \delta^{2} \mathbf{A}_{0}^{(2)} / \mu
$$

Therefore, we get that

$$
\begin{aligned}
\mathbf{L}(\delta)= & \sum_{\mu>0}\left(\mathbf{P}_{\mu} \mathbf{B}(\delta) \mathbf{P}_{0}+\mathbf{P}_{0} \mathbf{B}(\delta) \mathbf{P}_{\mu}\right) / \mu \\
& +\sum_{\mu>0} \frac{\mathbf{P}_{\mu} \mathbf{B}(\delta) \mathbf{P}_{0}}{\mu}\left(\left(\mathbf{I}-\delta^{2} \mathbf{A}_{0}^{(2)} / \mu\right)^{-1}-\mathbf{I}\right) \\
& +\sum_{\mu>0}\left(\left(\mathbf{I}-\delta^{2} \mathbf{A}_{0}^{(2)} / \mu\right)^{-1}-\mathbf{I}\right) \frac{\mathbf{P}_{0} \mathbf{B}(\delta) \mathbf{P}_{\mu}}{\mu} \\
= & \delta \mathbf{S}_{0}\left(\mathbf{H E}^{\mathrm{T}}+\mathbf{E H}^{\mathrm{T}}\right) \mathbf{P}_{0}+\delta \mathbf{P}_{0}\left(\mathbf{H} \mathbf{E}^{\mathrm{T}}+\mathbf{E} \mathbf{H}^{\mathrm{T}}\right) \mathbf{S}_{0} \\
& +\delta^{2}\left(\mathbf{S}_{0} \mathbf{A}^{(2)} \mathbf{P}_{0}+\mathbf{P}_{0} \mathbf{A}^{(2)} \mathbf{S}_{0}\right) \\
& +\sum_{\mu>0} \frac{\mathbf{P}_{\mu} \mathbf{B}(\delta) \mathbf{P}_{0}}{\mu} \frac{\delta^{2} \mathbf{A}_{0}^{(2)}}{\mu}\left(\mathbf{I}-\delta^{2} \mathbf{A}_{0}^{(2)} / \mu\right)^{-1} \\
& +\sum_{\mu>0}\left(\mathbf{I}-\delta^{2} \mathbf{A}_{0}^{(2)} / \mu\right)^{-1} \frac{\delta^{2} \mathbf{A}_{0}^{(2)}}{\mu} \frac{\mathbf{P}_{0} \mathbf{B}(\delta) \mathbf{P}_{\mu}}{\mu}
\end{aligned}
$$

Since $\mathbf{P}_{0} \mathbf{H}=\mathbf{0}$ and in view of the equality (2.9), we get the result.

\section{Proof of Theorem 2.6}

As shown in the demonstration of Theorem 2.5,

$$
\begin{aligned}
& \mathbf{P}_{0}^{\perp}(\delta)-\mathbf{P}_{0}^{\perp}-\mathbf{L}(\delta) \\
& \quad=\sum_{p=2}^{\infty}(-1)^{p} \sum_{\left(l_{1}, \ldots, l_{p+1}\right)}^{*} \mathbf{S}_{0}^{\left(l_{1}\right)} \mathbf{B}(\delta) \mathbf{S}_{0}^{\left(l_{2}\right)} \ldots \mathbf{B}(\delta) \mathbf{S}_{0}^{\left(l_{p+1}\right)},
\end{aligned}
$$

where $\sum_{\left(l_{1}, \ldots, l_{p+1}\right)}^{*}$ stands for the sum over all positive integers $l_{1}, \ldots, l_{p+1}$ such that $l_{1}+\cdots+l_{p+1}=p$ and $l_{1}, l_{p+1} \neq p$.

Let us calculate the sum

$$
\sum_{p=2}^{\infty}(-1)^{p} \sum_{\left(l_{1}, \ldots, l_{p+1}\right)}^{* *} \mathbf{S}_{0}^{\left(l_{1}\right)} \mathbf{B}(\delta) \mathbf{S}_{0}^{\left(l_{2}\right)} \ldots \mathbf{B}(\delta) \mathbf{S}_{0}^{\left(l_{p+1}\right)}
$$

where $\sum_{\left(l_{1}, \ldots, l_{p+1}\right)}^{* *}$ stands for the sum over all positive integers $l_{1}, \ldots, l_{p+1}$ with $l_{1}+\cdots+l_{p+1}=p$ such that for some $2 \leq k \leq p$ either the numbers $l_{1}, \ldots, l_{k}$ are positive and $l_{k+1}=\cdots=l_{p+1}=0$ or $l_{1}=\cdots=l_{p+1-k}=0$ and $l_{p+2-k}, \ldots, l_{p+1}>0$. Both variants are treated similarly.

Denote for brevity $\mathbf{M}_{j}=\left(\delta^{2} \mathbf{A}_{0}^{(2)} / \mu_{j}\right)^{l_{j}-1}$. If the numbers $l_{1}, \ldots, l_{k}$ are positive and $l_{j}=0$ for $j>k$, then 


$$
\begin{aligned}
& (-1)^{p} \mathbf{S}_{0}^{\left(l_{1}\right)} \mathbf{B}(\delta) \mathbf{S}_{0}^{\left(l_{2}\right)} \ldots \mathbf{B}(\delta) \mathbf{S}_{0}^{\left(l_{p+1}\right)} \\
& =(-1)^{k-1}\left(\prod_{j=1}^{k-1} \mathbf{S}_{0}^{l_{j}} \mathbf{B}(\delta)\right) \mathbf{S}_{0}^{l_{k}} \mathbf{B}(\delta) \mathbf{P}_{0}\left(\delta^{2} \mathbf{A}_{0}^{(2)}\right)^{p-k} \\
& =(-1)^{k-1}\left(\prod_{j=1}^{k-1} \sum_{\mu_{j}>0} \frac{\mathbf{P}_{\mu_{j}}}{\mu_{j}^{l_{j}}} \mathbf{B}(\delta)\right) \sum_{\mu_{k}} \frac{\mathbf{P}_{\mu_{k}} \mathbf{B}(\delta) \mathbf{P}_{0}}{\mu_{k}^{l_{k}}}\left(\delta^{2} \mathbf{A}_{0}^{(2)}\right)^{p-k} \\
& =(-1)^{k-1} \sum_{\mu_{1}, \ldots, \mu_{k}>0} \mathbf{J}_{k} \frac{\mathbf{P}_{\mu_{k}} \mathbf{B}(\delta) \mathbf{P}_{0}}{\mu_{k}} \frac{\left(\delta^{2} \mathbf{A}_{0}^{(2)}\right)^{p-k}}{\mu_{1}^{l_{1}-1} \ldots \mu_{k}^{l_{k}-1}} \\
& \sum_{\mu_{1}, \ldots, \mu_{k}>0} \mathbf{J}_{k} \frac{\mathbf{P}_{\mu_{k}} \mathbf{B}(\delta) \mathbf{P}_{0}}{\mu_{k}} \mathbf{M}_{k} \prod_{j=1}^{k-1} \mathbf{M}_{j} .
\end{aligned}
$$

For fixed $k \geq 2$ we have

$$
\begin{aligned}
& \sum_{p \geq k} \sum_{\substack{l_{1}+\ldots+l_{k}=p, l_{j}>0 \\
\mu_{1}, \ldots, \mu_{k}>0}} \mathbf{J}_{k} \frac{\mathbf{P}_{\mu_{k}} \mathbf{B}(\delta) \mathbf{P}_{0}}{\mu_{k}} \mathbf{M}_{k} \prod_{j=1}^{k-1} \mathbf{M}_{j} \\
& =\sum_{l_{1}, \ldots, l_{k}=1}^{\infty} \sum_{\mu_{1}, \ldots, \mu_{k}>0} \mathbf{J}_{k} \frac{\mathbf{P}_{\mu_{k}} \mathbf{B}(\delta) \mathbf{P}_{0}}{\mu_{k}} \mathbf{M}_{k} \prod_{j=1}^{k-1} \mathbf{M}_{j} \\
& =\sum_{\mu_{1}, \ldots, \mu_{k}>0} \mathbf{J}_{k} \frac{\mathbf{P}_{\mu_{k}} \mathbf{B}(\delta) \mathbf{P}_{0}}{\mu_{k}} \sum_{l_{k}=1}^{\infty} \mathbf{M}_{k} \prod_{j=1}^{k-1} \sum_{l_{j}=1}^{\infty} \mathbf{M}_{j} \\
& =\sum_{\mu_{1}, \ldots, \mu_{k-1}>0} \mathbf{J}_{k} \sum_{\mu_{k}} \frac{\mathbf{P}_{\mu_{k}} \mathbf{B}(\delta) \mathbf{P}_{0}}{\mu_{k}}\left(\mathbf{I}-\mathbf{M}_{k}\right)^{-1} \mathbf{G}_{k} \\
& =\sum_{\mu_{1}, \ldots, \mu_{k-1}>0} \mathbf{J}_{k} \mathbf{L}_{1}(\delta) \mathbf{G}_{k} .
\end{aligned}
$$

Since the case $k=1$ corresponds to $\mathbf{L}_{1}(\delta)$, then the sum of (6.9) over $k \geq 1$ gives us $\mathbf{T}_{1}$. The term $\mathbf{T}_{1}^{\mathrm{T}}$ corresponds to $l_{1}=\cdots=l_{p+1-k}=0$ and $l_{p+2-k}, \ldots, l_{p+1}>0$ and is calculated in the same manner.

Let us consider the residual $\mathbf{P}_{0}^{\perp}(\delta)-\mathbf{P}_{0}^{\perp}-\mathbf{T}(\delta)$. As was mentioned in the demonstration of Theorem 2.2, for any $l_{1}, \ldots, l_{p+1}$ with $l_{1}+\cdots+l_{p+1}=p$ there exists at least one $j$ such that $l_{j}>0$ and either $l_{j-1}=0$ or $l_{j+1}=0$. It is easy to understand that such $j$ is unique iff either $l_{i}>0$ for $i<j$ and $l_{i}=0$ for $i>j$ or $l_{i}=0$ for $i<j$ and $l_{i}>0$ for $i>j$.

This means that any term

$$
(-1)^{p} \mathbf{S}_{0}^{\left(l_{1}\right)} \mathbf{B}(\delta) \mathbf{S}_{0}^{\left(l_{2}\right)} \ldots \mathbf{B}(\delta) \mathbf{S}_{0}^{\left(l_{p+1}\right)}
$$

of the residual $\mathbf{P}_{0}^{\perp}(\delta)-\mathbf{P}_{0}^{\perp}-\mathbf{T}(\delta)$ corresponds to the vector $\left(l_{1}, \ldots, l_{p+1}\right)$ such that for some positive $l_{i}, l_{j}$ with $i<j$ each of the triples $\left(l_{i-1}, l_{i}, l_{i+1}\right),\left(l_{j-1}, l_{j}, l_{j+1}\right)$ contains a unique zero. Denote

$m=\left\{\begin{array}{ll}i & \text { for } l_{i+1}=0, \\ i-1 & \text { for } l_{i-1}=0\end{array}\right.$ and $k= \begin{cases}j & \text { for } l_{j+1}=0 \\ j-1 & \text { for } l_{j-1}=0\end{cases}$

and consider the pairs $(m, m+1)$ and $(k, k+1)$. Note that these pairs are either disjoint or $m+1=i+1=k=j-1$ with $l_{m+1}=l_{k}=0$. If pairs are disjoint, then

$$
\begin{aligned}
& \left\|\mathbf{S}_{0}^{\left(l_{1}\right)} \mathbf{B}(\delta) \mathbf{S}_{0}^{\left(l_{2}\right)} \ldots \mathbf{B}(\delta) \mathbf{S}_{0}^{\left(l_{p+1}\right)}\right\| \\
& =\left\|\mathbf{S}_{0}^{\left(l_{1}\right)} \ldots \mathbf{S}_{0}^{\left(l_{m}\right)} \mathbf{B}(\delta) \mathbf{S}_{0}^{\left(l_{m+1}\right)} \ldots \mathbf{S}_{0}^{\left(l_{k}\right)} \mathbf{B}(\delta) \mathbf{S}_{0}^{\left(l_{k+1}\right)} \ldots \mathbf{S}_{0}^{\left(l_{p+1}\right)}\right\| \\
& \leq\left\|\mathbf{S}_{0} \mathbf{B}(\delta) \mathbf{P}_{0}\right\|^{2}\left(\|\mathbf{B}(\delta)\| / \mu_{\min }\right)^{p-2} .
\end{aligned}
$$

If $m+1=k$, then $l_{m}, l_{m+2}>0$ and

$$
\begin{aligned}
& \left\|\mathbf{S}_{0}^{\left(l_{1}\right)} \mathbf{B}(\delta) \mathbf{S}_{0}^{\left(l_{2}\right)} \ldots \mathbf{B}(\delta) \mathbf{S}_{0}^{\left(l_{p+1}\right)}\right\| \\
& =\left\|\mathbf{S}_{0}^{\left(l_{1}\right)} \mathbf{B}(\delta) \ldots \mathbf{S}_{0}^{\left(l_{m}\right)} \mathbf{B}(\delta) \mathbf{P}_{0} \mathbf{P}_{0} \mathbf{B}(\delta) \mathbf{S}_{0}^{\left(l_{m+2}\right)} \ldots \mathbf{B}(\delta) \mathbf{S}_{0}^{\left(l_{p+1}\right)}\right\| \\
& \leq\left\|\mathbf{S}_{0} \mathbf{B}(\delta) \mathbf{P}_{0}\right\|^{2}\left(\|\mathbf{B}(\delta)\| / \mu_{\min }\right)^{p-2} .
\end{aligned}
$$

The rest of proof is the same as in Theorem 2.5.

\section{Proof of Proposition 3.1}

If $\Theta \rightarrow 0$, then $\Theta_{1} \rightarrow 0$ and

$$
\|\mathbf{B}(\delta)\| / \mu_{\min } \leq 2|\delta| \Theta+\delta^{2} \Theta \Theta_{1} \sim 2|\delta| \Theta \rightarrow 0
$$

for all $\delta$. This means that we can use the inequalities (2.16) and (2.24) for any fixed $\delta$ provided that $N$ is sufficiently big. The inequality (3.1) follows from (2.16), (2.17). In the same manner the inequalities $(2.22),(2.17)$ imply

$$
\underset{N}{\limsup } \Theta^{-2}\left\|\mathbf{P}_{0}^{\perp}(\delta)-\mathbf{P}_{0}^{\perp}-\mathbf{W}_{1}(\delta)\right\| \leq 64 C \delta^{2} .
$$

Since $\mathbf{W}_{1}(\delta)=\delta \mathbf{V}_{0}^{(1)}+\delta^{2}\left(\mathbf{P}_{0} \mathbf{A}^{(2)} \mathbf{S}_{0}+\mathbf{S}_{0} \mathbf{A}^{(2)} \mathbf{P}_{0}\right)$ and $\left\|\mathbf{S}_{0} \mathbf{A}^{(2)} \mathbf{P}_{0}\right\|=\left\|\mathbf{P}_{0} \mathbf{A}^{(2)} \mathbf{S}_{0}\right\| \leq \Theta \Theta_{1} \leq \Theta^{2}$, then (3.2) is proved as well.

\section{Proof of Proposition 3.2}

It follows from the convergence $\left\|\mathbf{H E}^{\mathrm{T}}\right\| / \mu_{\min } \rightarrow 0$ that

$$
\begin{aligned}
& \limsup \|\mathbf{B}(\delta)\| / \mu_{\min } \\
& \leq 2|\delta| \lim \sup \left\|\mathbf{H E}^{\mathrm{T}}\right\| / \mu_{\min } \\
& +\delta^{2} \lim \sup \Theta \Theta_{1}=\delta^{2} / \Delta<1 / 4
\end{aligned}
$$

Therefore, (2.20) holds for $|\delta|<\delta_{0}$ and big $N$. Moreover, $1 /\left(1-4\|\mathbf{B}(\delta)\| / \mu_{\min }\right) \leq 1 /(1-4 \Delta)$. Thus (3.3) follows from (2.20) and (6.7). Applying (2.24) instead of (2.20), we get (3.4).

\section{Proof of Proposition 3.3}

In view of Lemma 3.1, $\mu_{\max } \sim T_{\max }^{(a)} a_{1}^{2 N}$ and $\mu_{\min } \sim$ $T_{\min }^{(a)} a_{p}^{2 N}$. Therefore,

$$
\begin{aligned}
\Theta_{1} & =\sqrt{\nu_{\max } / \mu_{\max }} \sim \sqrt{\nu_{\max } / T_{\max }^{(a)}} a_{1}^{-N}, \\
\Theta_{2} & =\mu_{\max } / \mu_{\min } \sim\left(T_{\max }^{(a)} / T_{\min }^{(a)}\right)\left(a_{1} / a_{p}\right)^{2 N}, \\
\Theta & =\Theta_{1} \Theta_{2} \sim \sqrt{\nu_{\max }},\left(a_{1} / a_{p}^{2}\right)^{N} \Xi_{a}
\end{aligned}
$$

and both assertions follow from Proposition 3.1. 


\section{Proof of Proposition 4.1}

We present only the sketch of the proof. Note that the righthand side of (4.2) is $O\left(N^{2} a^{-2 N}\right)$ if $L / N \rightarrow \alpha \in(0,1)$ and has the order $O\left(N a^{-2 N}\right)$ in the case $\min (L, K)=$ const $>1$. Let us study the asymptotic behavior of $\mathbf{V}_{0}^{(1)}$.

Accurate calculations show that

$$
\begin{aligned}
\left\|\mathbf{V}_{0}^{(1)}\right\| & \sim\left\|\mathbf{Z}_{0}^{(1)}\right\| \\
& \sim \frac{\sqrt{N}}{a^{N+1}} \begin{cases}\alpha(a+1) \sqrt{a^{2}-1} & \text { for } L / N \rightarrow \alpha \in(0,1), \\
\frac{(a+1) \sqrt{a^{2}-1}}{1-a^{-K_{0}}} & \text { for } K=K_{0}>1\end{cases}
\end{aligned}
$$

and

$$
\left\|\mathbf{V}_{0}^{(1)}\right\| \sim \frac{(a+1)}{a} \frac{a^{L_{0}} \sqrt{L_{0}\left\|W_{L_{0}}\right\|^{2}-\beta_{L_{0}}^{2}}}{\left\|W_{L_{0}}\right\|^{2}} a^{-N}
$$

for $L=L_{0}=$ const $>1$. This completes the proof.

\section{Proof of Proposition 4.2}

Simple calculations show that $\left\|\mathbf{H E}^{\mathrm{T}}\right\| / \mu_{\min }=0$ if $K$ is even and that $\left\|\mathbf{H E}^{\mathrm{T}}\right\| / \mu_{\min }=1 / K$ for odd $K$. Additionally, $\left\|\mathbf{S}_{0} \mathbf{A}^{(2)}\right\|=0$ for even $L$ and $\left\|\mathbf{S}_{0} \mathbf{A}^{(2)}\right\|=1 / L$ for odd $L$.

The assertion of Theorem 2.5 holds under the condition $2|\delta|\left\|\mathbf{H E}^{\mathrm{T}}\right\|+\delta^{2} \nu_{\max }<\mu_{\min } / 4$. If $K$ is even, then this inequality is equivalent to $\delta^{2}<1 / 4$. If $K$ is odd and tends to infinity, then we asymptotically get the same restriction $\delta^{2}<1 / 4$. Since the right-hand side of (3.3) has the form $O\left(\left\|\mathbf{H E}^{\mathrm{T}}\right\| / \mu_{\min }+\left\|\mathbf{S}_{0} \mathbf{A}^{(2)}\right\|\right)$, the proof is complete.

\section{Proof of Proposition 4.3}

The first assertion follows from the equality $\left\|W_{E}\right\|=$ $\sqrt{L^{2}-\beta_{L}}$.

Let us prove the second assertion. Straightforward calculations show that

$$
\left\|\mathbf{S}_{0} \mathbf{B}(\delta)\right\|\left\|\mathbf{S}_{0} \mathbf{B}(\delta) \mathbf{P}_{0}\right\| \leq \delta^{2}\left(\frac{\beta_{K}}{K}+\frac{\beta_{L} \beta_{K}}{L K}+|\delta| \frac{\beta_{L}}{L}\right)^{2}
$$

and

$$
\|\mathbf{B}(\delta)\| / \mu_{\min } \leq|\delta| \beta_{k} \sqrt{L^{2}-\beta_{L}} / L K+\delta^{2} \leq \beta_{k}|\delta| / K+\delta^{2} .
$$

Therefore, it follows from (2.24) that for any $|\delta|<1 / 2$

$$
\begin{aligned}
& \left\|\mathbf{P}_{0}^{\perp}(\delta)-\mathbf{P}_{0}^{\perp}-\mathbf{L}(\delta)\right\| \\
& \quad= \begin{cases}O\left(K^{-2}\right) & \text { for odd } K \rightarrow \infty \text { and even } L, \\
O\left(L^{-2}\right) & \text { for odd } L \rightarrow \infty \text { and even } K, \\
O\left(L^{-2}+K^{-2}\right) & \text { for odd } K, L\end{cases} \\
& \text { and } \min (L, K) \rightarrow \infty .
\end{aligned}
$$

Comparing $\mathbf{L}(\delta)$ with $\mathbf{M}(\delta)$ we see that $\mathbf{L}(\delta)=\mathbf{M}(\delta)$ in the case when $L$ is even and $K$ is odd. Otherwise,

$$
\begin{aligned}
\mathbf{L}(\delta)= & \mathbf{M}(\delta) \\
& +\left\{\begin{array}{l}
O\left(L^{-3}\right) \\
O\left(L^{-2} K^{-1}+L^{-3}\right)
\end{array}\right.
\end{aligned}
$$

for odd $L \rightarrow \infty$ and even $K$, for odd $K, L$ and $\min (L, K) \rightarrow \infty$.

This completes the proof.

\section{Proof of Proposition 5.1}

Note that

$$
\begin{aligned}
R(\delta)-R= & -\mathbf{G}_{L} \frac{1}{\left\|\mathbf{P}_{0}(\delta) \mathfrak{e}_{L}\right\|^{2}}\left(\mathbf{P}_{0}(\delta)-\mathbf{P}_{0}\right) \mathfrak{e}_{L} \\
& +\mathbf{G}_{L}\left(\frac{1}{\left\|\mathbf{P}_{0}(\delta) \mathfrak{e}_{L}\right\|^{2}}-\frac{1}{\left\|\mathbf{P}_{0} \mathfrak{e}_{L}\right\|^{2}}\right) \mathbf{P}_{0} \mathfrak{e}_{L} \\
= & J_{1}+J_{2} .
\end{aligned}
$$

Since $\left\|\mathbf{G}_{L}\right\|=1, \mathbf{P}_{0}(\delta)-\mathbf{P}_{0}=\mathbf{P}_{0}^{\perp}-\mathbf{P}_{0}^{\perp}(\delta)$, and $\left\|\mathbf{P}_{0}(\delta) \mathfrak{e}_{L}\right\| \geq\left\|\mathbf{P}_{0} \mathfrak{e}_{L}\right\|-\Delta \mathbf{P}(\delta)$, then

$$
\left\|\mathbf{P}_{0}(\delta) \mathfrak{e}_{L}\right\| /\left\|\mathbf{P}_{0} \mathfrak{e}_{L}\right\| \geq 1-\Delta \mathbf{P}(\delta) /\left\|\mathbf{P}_{0} \mathfrak{e}_{L}\right\|,
$$

$$
\left\|J_{1}\right\| \leq \frac{\Delta \mathbf{P}(\delta)}{\left\|\mathbf{P}_{0} \mathfrak{e}_{L}\right\|^{2}}\left(\frac{\left\|\mathbf{P}_{0} \mathfrak{e}_{L}\right\|}{\left\|\mathbf{P}_{0}(\delta) \mathfrak{e}_{L}\right\|}\right)^{2} \leq \frac{\Delta \mathbf{P}(\delta)}{1-\vartheta^{2}}\left(1-\frac{\Delta \mathbf{P}(\delta)}{\sqrt{1-\vartheta^{2}}}\right)^{-2}
$$

and

$$
\begin{aligned}
\left\|J_{2}\right\| & \leq \frac{1}{\left\|\mathbf{P}_{0} \mathfrak{e}_{L}\right\|^{2}}\left(\frac{\left\|\mathbf{P}_{0} \mathfrak{e}_{L}\right\|^{2}}{\left\|\mathbf{P}_{0}(\delta) \mathfrak{e}_{L}\right\|^{2}}-1\right) \\
& \leq \frac{1}{\left\|\mathbf{P}_{0} \mathfrak{e}_{L}\right\|^{2}}\left(\frac{1}{\left(1-\Delta \mathbf{P}(\delta) /\left\|\mathbf{P}_{0} \mathfrak{e}_{L}\right\|\right)^{2}}-1\right) \\
& \leq \frac{2 \Delta \mathbf{P}(\delta)}{\left(1-\vartheta^{2}\right)^{3 / 2}}\left(1-\frac{\Delta \mathbf{P}(\delta)}{\sqrt{1-\vartheta^{2}}}\right)^{-2} .
\end{aligned}
$$

Thus (5.5) is proved.

\section{Proof of Lemma 5.1}

1. Denote $\mathbf{U}=\left[U_{1}: \ldots: U_{d}\right]$. Since $U_{1}, \ldots, U_{d}$ are linearly independent, then $\mathbf{P} \mathbf{U} X=\mathbf{U} X \neq 0$ for any $X \neq 0$. Consider the vector $\mathbf{Q} \mathbf{U} X=(\mathbf{Q}-\mathbf{P}) \mathbf{U} X+\mathbf{U} X$. Then

$$
\begin{aligned}
\|\mathbf{Q U} X\| & \geq\|\mathbf{U} X\|-\|\mathbf{Q}-\mathbf{P}\|\|\mathbf{U} X\| \\
& =(1-\|\mathbf{Q}-\mathbf{P}\|)\|\mathbf{U} X\|>0 .
\end{aligned}
$$

2. Consider the linear space $\mathbf{P} \mathbb{V}$. Evidently, $\mathbf{P} \mathbb{V}$ is a subspace of $\mathbb{U}$. Due to the first assertion, the dimension of $\mathbf{P} \mathbb{V}$ equals $d$. Therefore, $\mathbf{P} \mathbb{V}=\mathbb{U}$. This completes the proof.

\section{Proof of Proposition 5.2}

Denote for short $\mathbf{X}=\mathbf{U}^{\mathrm{T}} \mathbf{F}_{1} \mathbf{U}, \mathbf{Y}=\mathbf{U}^{\mathrm{T}} \mathbf{F}_{2} \mathbf{U}, \mathbf{X}(\delta)=$ $\mathbf{U}^{\mathrm{T}} \mathbf{P}_{0}^{\perp}(\delta) \mathbf{F}_{1} \mathbf{P}_{0}^{\perp}(\delta) \mathbf{U}$, and $\mathbf{Y}(\delta)=\mathbf{U}^{\mathrm{T}} \mathbf{P}_{0}^{\perp}(\delta) \mathbf{F}_{2} \mathbf{P}_{0}^{\perp}(\delta) \mathbf{U}$. 
Note that $\|\mathbf{Y}(\delta)\| \leq\|\mathbf{U}\|^{2}$. Then

$$
\begin{aligned}
\widehat{\mathbf{D}}(\delta)-\mathbf{D} & =\mathbf{X}^{-1}(\delta) \mathbf{Y}(\delta)-\mathbf{X}^{-1} \mathbf{Y} \\
& =\left(\mathbf{X}^{-1}(\delta)-\mathbf{X}^{-1}\right) \mathbf{Y}(\delta)+\mathbf{X}^{-1}(\mathbf{Y}(\delta)-\mathbf{Y}) \\
& =J_{1}+J_{2} .
\end{aligned}
$$

Since $\mathbf{Y}(\delta)-\mathbf{Y}=\mathbf{U}^{\mathrm{T}} \Delta \mathbf{P}(\delta) \mathbf{F}_{2} \mathbf{P}_{0}^{\perp}(\delta) \mathbf{U}+\mathbf{U}^{\mathrm{T}} \mathbf{P}_{0}^{\perp} \mathbf{F}_{2} \Delta \mathbf{P}(\delta) \mathbf{U}$, then $\|\mathbf{Y}(\delta)-\mathbf{Y}\| \leq 2\|\mathbf{U}\|^{2} \Delta \mathbf{P}(\delta)$ and we obtain the inequality

$$
\left\|J_{2}\right\| \leq \frac{2\|\mathbf{U}\|^{2}}{\left\|\mathbf{U}^{\mathrm{T}} \mathbf{F}_{1} \mathbf{U}\right\|} \Delta \mathbf{P}(\delta)=\frac{2}{v} \Delta \mathbf{P}(\delta)
$$

In the same manner, $\|\mathbf{X}(\delta)-\mathbf{X}\| \leq 2\|\mathbf{U}\|^{2} \Delta \mathbf{P}(\delta)$ and $\left\|\mathbf{X}^{-1}(\mathbf{X}(\delta)-\mathbf{X})\right\| \leq 2 \Delta \mathbf{P}(\delta) / v<1$. Therefore,

$$
\mathbf{X}^{-1}(\delta)-\mathbf{X}^{-1}=\left(\sum_{j=1}^{\infty}\left(\mathbf{I}+\mathbf{X}^{-1}(\mathbf{X}(\delta)-\mathbf{X})\right)^{j}\right) \mathbf{X}^{-1}
$$

and

$$
\begin{aligned}
& \left\|\left(\mathbf{X}^{-1}(\delta)-\mathbf{X}^{-1}\right) \mathbf{Y}(\delta)\right\| \\
& \leq \frac{\|\mathbf{Y}(\delta)\|}{\|\mathbf{X}\|} \frac{\left\|\mathbf{X}^{-1}(\mathbf{X}(\delta)-\mathbf{X})\right\|}{1-\left\|\mathbf{X}^{-1}(\mathbf{X}(\delta)-\mathbf{X})\right\|} \\
& \leq \frac{2}{v^{2}} \frac{\Delta \mathbf{P}(\delta)}{1-2 \Delta \mathbf{P}(\delta) / v}
\end{aligned}
$$

The proof is complete.

\section{Proof of Proposition 5.3}

The first assertion follows from Remark 5.2. Let us prove the assertions $2-4$. Since $\|\mathbf{H}\|=\|\mathbf{E}\|=\sqrt{L K}$ and in view of (4.6),

$$
\begin{aligned}
& \left\|\left(\mathbf{P}_{0}^{\perp}(\delta)-\mathbf{P}_{0}^{\perp}-\mathbf{M}(\delta)\right) \mathbf{H}(\delta)\right\| \\
& = \begin{cases}O\left(\sqrt{L / K^{3}}\right) & \text { for odd } K, \text { even } L, \\
O\left(\sqrt{K / L^{3}}\right) & \text { and } L=o\left(K^{3}\right), \\
O\left(\sqrt{L / K^{3}}+\sqrt{K / L^{3}}\right) & \text { for odd } L, \text { even } K, \\
\min \left(L / K^{3}, K / L^{3}\right) \rightarrow \infty .\end{cases}
\end{aligned}
$$

This means that we must study the entries of the matrix $\mathcal{S}\left(\mathbf{M}(\delta) \mathbf{H}(\delta)+\delta \mathbf{P}_{0}^{\perp} \mathbf{E}\right)$. Calculations show that under denotation $V_{E}=W_{L} E_{K}^{\mathrm{T}}$ and $W_{E}(\delta)=E_{L} W_{K}^{\mathrm{T}}+\delta V_{E}$,

$$
\begin{aligned}
& \mathbf{M}(\delta) \mathbf{H}(\delta)+\delta \mathbf{P}_{0}^{\perp} \mathbf{E} \\
& =\left\{\begin{array}{l}
\frac{\delta}{1-\delta^{2}} \frac{W_{E}(\delta)}{K} \\
\frac{\delta^{2}}{1-\delta^{2}} \frac{W_{E}(\delta)}{L}+\delta \frac{V_{E}}{L}+O\left(L^{-2}\right) \\
\frac{\delta}{1-\delta^{2}}\left(\frac{1}{K}+\frac{\delta}{L}\right)\left(W_{E}(\delta)+O\left(L^{-1}\right)\right)+\delta \frac{V_{E}}{L}
\end{array}\right.
\end{aligned}
$$

for odd $K$ and even $L$, for odd $L$ and even $K$, for $K, L$ both odd.

Now the result becomes clear.

\section{Proof of Proposition 5.4}

Let us consider the case $L / N \rightarrow \alpha \in(0,1)$ (other cases are studied in the same manner). It is easy to see that

$$
\begin{aligned}
\Lambda\left(\delta \mathbf{V}_{0}^{(1)}\right) & =\delta\left(\mathbf{V}_{0}^{(1)} \mathbf{H}+\mathbf{P}_{0}^{\perp} \mathbf{E}\right)+\delta^{2} \mathbf{V}_{0}^{(1)} \mathbf{E} \\
& =\Lambda_{1}\left(\delta \mathbf{V}_{0}^{(1)}\right)+O\left(N^{3 / 2} a^{-N}\right)
\end{aligned}
$$

and

$$
\Lambda_{1}\left(\delta \mathbf{V}_{0}^{(1)}\right)=\delta\left(-\frac{\beta_{L} \beta_{K} \mathbf{H}}{\left\|W_{L}\right\|^{2}\left\|W_{K}\right\|^{2}}+\frac{\beta_{K} E_{L} W_{K}^{\mathrm{T}}}{\left\|W_{K}\right\|^{2}}+\frac{\beta_{L} W_{L} E_{K}^{\mathrm{T}}}{\left\|W_{L}\right\|^{2}}\right) .
$$

Therefore,

$$
\left\|\widetilde{\mathrm{F}}_{N}(\delta)-\mathrm{F}_{N}\right\|_{\max }=\lambda_{1}\left(\delta \mathbf{V}_{0}^{(1)}\right)+O\left(N^{2} a^{-N}\right),
$$

where $\lambda_{1}\left(\delta \mathbf{V}_{0}^{(1)}\right)=\left\|\mathcal{S} \Lambda_{1}\left(\delta \mathbf{V}_{0}^{(1)}\right)\right\|_{\max }$.

Consider the series $g_{0}, \ldots, g_{N-1}$ corresponding to the Hankel matrix $\mathcal{S} \Lambda_{1}\left(\delta \mathbf{V}_{0}^{(1)}\right)$. Since hankelization does not modify extreme entries $a_{00}$ and $a_{n-1 m-1}$ of any $n \times m$ matrix $\mathbf{A}=\left\{a_{i j}\right\}_{i=0, j=0}^{n, m}$, then it is easy to see that

$$
\begin{aligned}
g_{N-1}= & \delta\left(-\frac{\beta_{L} \beta_{K}}{\left\|W_{L}\right\|^{2}\left\|W_{K}\right\|^{2}} a^{N-1}\right. \\
& \left.+\frac{\beta_{K}}{\left\|W_{K}\right\|^{2}} a^{K-1}+\frac{\beta_{L}}{\left\|W_{L}\right\|^{2}} a^{L-1}\right) \\
& \rightarrow \delta\left(a^{2}-1\right) / a^{2} \neq 0 .
\end{aligned}
$$

Thus the proof is complete.

\section{ACKNOWLEDGEMENTS}

The author is grateful to the anonymous referee and to the guest editor for many helpful comments.

\section{Received 03 December 2009}

\section{REFERENCES}

[1] DE Prony, G. S. (1795). Essai experimental et analytique: sur les lois de la dilatabilite de fluides elastique et sur celles de la force expansive de la vapeur de l'alkool, a differentes temperatures. Journal de l'Ecole Polytechnique 1(22) 24-76.

[2] Fraedrich, K. (1986). Estimating the dimension of weather and climate attractors. J. Atmos. Sci. 43 419-432. MR0838629

[3] Weare B. C. and Nasstrom J. S. (1982). Examples of Extended Empirical Orthogonal Functions. Monthly Weather Review 110 481-485.

[4] Cadzow, J. A. (1988). Signal Enhancement - A Composite Property Mapping Algorithm. IEEE Transactions on Acoustics, Speech and Signal Processing 36(1) 49-62.

[5] Belonin M. D. et al. (1971). Factor Analysis in Petrolium Geology. (Review). VIEMS, Moscow. (In Russian).

[6] Colebrook, J. M. (1978). Continuous plankton records - zooplankton and environment, northeast Atlantic and North Sea, 1948-1975. Oceanol. Acta 1 9-23.

[7] Basilevsky, A. And Hum, P. J. (1979) Karhunen-Loève analysis of historical time series with application to Plantation birth in Jamaica. Journal of the American Statistical Association 74284 290. 
[8] Efimov, V. M. and Galaktionov, Yu. K. (1983). On the Prediction of Cyclical Variations of Mammal Populations. Russian Journal of General Biology 3 343-352. (In Russian).

[9] Kumaresan, R. and Tuffs, D. (1980). Data-adaptive principal component signal processing. In: IEEE Proc. Conf. on Decision and Control, IEEE, Albuquerque, USA, 949-954.

[10] Broomhead D. S. And KInG, G. P. (1986). Extracting qualitative dynamics from experimental data. Physica D 20 217-236. MR0859354

[11] Ku, W., Storer, R. and Georgakis, C. (1995). Disturbance detection and isolation by dynamic principal component analysis, Chemom. Intell. Lab. Sys. 30 179-196.

[12] Vautard, R., Yiou, P. and Ghil, M. (1992). Singular-spectrum analysis: A toolkit for short, noisy chaotic signals. Physica D 58 95-126.

[13] Golyandina, N., Nekrutkin, V. and Zhigljavsky, A. (2001). Analysis of Time Series Structure. SSA and Related Techniques. Champan \& Hall/CRC, Boca Raton-London-New YorkWashington D.C. MR1823012

[14] Buchstaber, V. M. (1994). Time series analysis and grassmannians. In: Applied Problems of Radon Transform. Ed. S. Gindikin. Providence, RI: AMS, 1-17 (Ser. AMS Transactions - Series 2, vol. 162.) MR1305833

[15] Marple, S. L. (1987). Digital Spectral Analysis with Applications. Prentice-Hall, New Jersey. MR0920370

[16] Schmidt, R. O. (1986). Multiple emitter location and signal parameter estimation. In: Proc. RADC Spectrum Estimation Workshop, Rome, NY. IEEE Transactions on Antennas and Propagation AP-34(2) 276-280.

[17] Roy, R. AND KaIlath, T. (1989). ESPRIT - estimation of signal parameters via rotational invariance techniques. IEEE Transactions on Acoustics, Speech and Signal Processing 37(7) 984995. MR1058066
[18] Badeau, R., Richard, G. And David B. (2008). Performance of ESPRIT for Estimating Mixtures of Complex Exponentials Modulated by Polynomials. IEEE Trans. Signal Processing 56(2) 492504. MR2445532

[19] Akhiezer, N. I. And Glazman, I. M. (1993). Theory of Linear Operators in Hilbert Space. Dover Publications Inc., New York. MR1255973

[20] Kato, T. (1966). Perturbation theory for linear operators. Springer-Verlag, Berlin-Heidelberg-New York. MR0203473

[21] Nekrutkin, V. (2010). Perturbation expansions of signal subspaces for long signals. Preprint available at http://arxiv.org/abs/ 1001.1051.

[22] Kumaresan, R. and Tufts, D. W. (1983). Estimating the angles of arrival of multiple plane waves. IEEE Transactions on Aerospace and Electronic Systems AES-19 (1) 134-139.

[23] Golyandina, N. (2010). On the choice of parameters in Singular Spectrum Analysis and related subspace-based methods. Statistics and Its Interface 3 259-279.

[24] Golub, G. and Van Loan, Ch. F. (1996). Matrix Computations. Third Edition. The Johns Hopkins University Press, Baltimore. MR1417720

\section{Vladimir Nekrutkin}

Math. Department, St. Petersburg University

Universitetsky av. 28

198504, St. Petersburg, Petrodvorets

Russia

E-mail address: vnekr@mail.ru 